NBER WORKING PAPER SERIES

\title{
MONETARY POLICY WITH MODEL UNCERTAINTY: DISTRIBUTION FORECAST TARGETING
}

\author{
Lars E. O. Svensson \\ Noah Williams \\ Working Paper 11733 \\ http://www.nber.org/papers/w11733
NATIONAL BUREAU OF ECONOMIC RESEARCH
1050 Massachusetts Avenue
Cambridge, MA 02138
November 2005

We thank Pierpaolo Benigno, Marvin Goodfriend, Boris Hoffman, Eric Leeper, Rujikorn Pavasuthipaisit, and participants in the New York Area Workshop on Monetary Policy, New York, May 2005, the Conference on Macroeconomic Risk and Policy Responses, Berlin, May 2005, and the Conference on Computing in Economics and Finance, Washington, DC, June 2005, for helpful comments. Expressed views and any remaining errors are our own responsibility. The views expressed herein are those of the author(s) and do not necessarily reflect the views of the National Bureau of Economic Research.

(C2005 by Lars E. O. Svensson and Noah Williams. All rights reserved. Short sections of text, not to exceed two paragraphs, may be quoted without explicit permission provided that full credit, including (C) notice, is given to the source. 
Monetary Policy with Model Uncertainty: Distribution Forecast Targeting

Lars E. O. Svensson and Noah Williams

NBER Working Paper No. 11733

November 2005

JEL No. E42, E52, E58

\section{$\underline{\text { ABSTRACT }}$}

We examine optimal and other monetary policies in a linear-quadratic setup with a relatively general form of model uncertainty, so-called Markov jump-linear-quadratic systems extended to include forward-looking variables. The form of model uncertainty our framework encompasses includes: simple i.i.d. model deviations; serially correlated model deviations; estimable regime-switching models; more complex structural uncertainty about very different models, for instance, backwardand forward-looking models; time-varying central-bank judgment about the state of model uncertainty; and so forth. We provide an algorithm for finding the optimal policy as well as solutions for arbitrary policy functions. This allows us to compute and plot consistent distribution forecasts--fan charts---of target variables and instruments. Our methods hence extend certainty equivalence and "mean forecast targeting" to more general certainty non-equivalence and "distribution forecast targeting."

Lars E. O. Svensson

Department of Economics

Fisher Hall

Princeton University

Princeton, NJ 08544-1021

and NBER

svensson@princeton.edu

Noah Williams

Department of Economics

001 Fisher Hall

Princeton University

Princeton, NJ 08544-1921

and NBER

noahw@princeton.edu 


\section{Contents}

1 Introduction $\quad 1$

2 The model $\quad 4$

2.1 The baseline model . . . . . . . . . . . . . . . . . . . . 4

2.2 Reformulation according to the recursive saddlepoint method . . . . . . . . . 6

2.3 Optimal policy and dynamics . . . . . . . . . . . . . . . 7

3 Interpretation of model uncertainty in our framework $\quad 9$

4 Examples $\quad 11$

4.1 An estimated backward-looking model . . . . . . . . . . . . . . . . . . 11

4.2 An estimated forward-looking model . . . . . . . . . . . . . . . . . . . 14

5 Arbitrary time-varying instrument rules and instrument paths 19

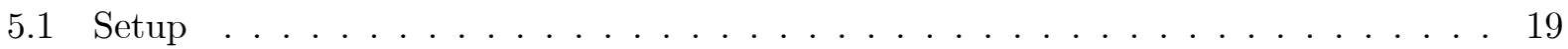

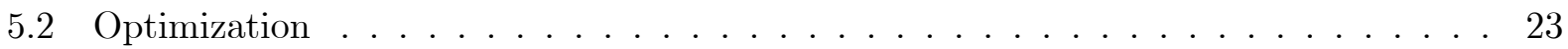

6 Arbitrary time-invariant instrument rules and optimal restricted instrument $\begin{array}{ll}\text { rules } & 23\end{array}$

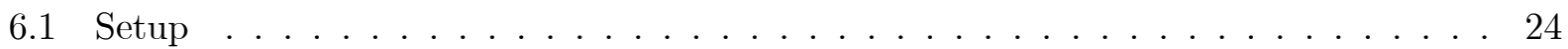

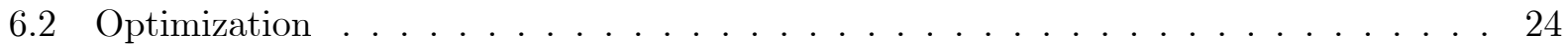

6.3 Optimal Taylor-type instrument rules in a forward-looking model . . . . . . . . . . 25

7 Unobservable modes $\quad 27$

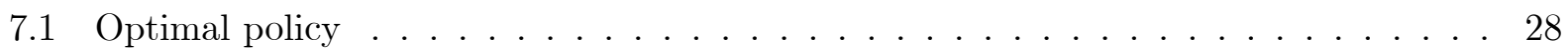

7.2 Examples . . . . . . . . . . . . . . . . . . . . 29

8 Conclusions $\quad 32$

$\begin{array}{ll}\text { Appendix } & 35\end{array}$

A Incorporating central-bank judgment 35

B An algorithm for the value function and optimal policy function 36

$\begin{array}{ll}\text { C A unit discount factor } & 39\end{array}$

$\begin{array}{ll}\text { D Mean square stability } & 40\end{array}$

E Alternative models with different predetermined and forward-looking variables 41

F Details of the estimation $\quad 43$

G Details for arbitrary time-varying instrument rules $\quad 44$

H Details for arbitrary time-invariant instrument rules $\quad 45$ 
I Details with unobservable modes $\quad 48$

I.1 Unobservable modes and forward-looking variables . . . . . . . . . . . . . 48

I.2 An algorithm for the model with forward-looking variables . . . . . . . . . . . . 51

I.3 Unobservable modes without forward-looking variables . . . . . . . . . . . . . . 53

I.4 An algorithm for the backward-looking model . . . . . . . . . . . . . . . 54

J Optimization under discretion 


\section{Introduction}

In recent years there has been a renewed interest in the study of optimal monetary policy under uncertainty. Classical analyses of optimal policy consider only additive sources of uncertainty, where in a linear-quadratic framework the well-known certainty-equivalence result applies and implies that optimal policy is the same as if there were no uncertainty. Recognizing the uncertain environment that policymakers face, recent research has considered broader forms of uncertainty for which certainty equivalence no longer applies. While this may have important implications, in practice the design of policy becomes much more difficult outside the classical linear-quadratic framework.

One of the conclusions of the Onatski and Williams [28] study of model uncertainty is that, for progress to be made, the structure of the model uncertainty has to be explicitly modeled. In line with this, in this paper we develop a very explicit but still relatively general form of model uncertainty that remains quite tractable, using a so-called Markov jump-linear-quadratic (MJLQ) model, where model uncertainty takes the form of different "modes" that follow a Markov process. Our approach allows us to move beyond the classical linear-quadratic world with additive shocks, yet remains close enough to the linear-quadratic framework that the analysis is transparent. We examine optimal and other monetary policies in an extended linear-quadratic setup, extended in a way to capture model uncertainty. The forms of model uncertainty our framework encompasses include: simple i.i.d. model deviations; serially correlated model deviations; estimable regime-switching models; more complex structural uncertainty about very different models, for instance, backward- and forward-looking models; time-varying central-bank judgment-information, knowledge, and views outside the scope of a particular model (Svensson [36]) - about the state of model uncertainty; and so forth. Moreover, while we focus on model uncertainty, our methods also apply to other linear models with changes of regime which may capture boom/bust cycles, productivity slowdowns and accelerations, switches in monetary and/or fiscal policy regimes, and so forth. We provide an algorithm for finding the optimal policy as well as solutions for arbitrary policy functions. This allows us to compute and plot consistent distribution forecasts - fan charts - of target variables and instruments. Our methods hence extend certainty equivalence and "mean forecast targeting," where only the mean of future variables matter (Svensson [36]), to more general certainty non-equivalence and "distribution forecast targeting," where the whole probability distribution of future variables matter (Svensson [35]). ${ }^{1}$

\footnotetext{
${ }^{1}$ The importance of the whole distribution of future target variables was recently emphasized by Greenspan [18] at the 2005 Jackson Hole symposium, with reference to his [17] so-called risk-management approach:
} 
Certain aspects of our approach have been known in economics since the classic works of Aoki [2] and Chow [8], who allowed for multiplicative uncertainty in a linear-quadratic framework. The insight of those papers, when adapted to our setting, is that in MJLQ models the value function remains quadratic in the state, but now with weights that depend on the mode. MJLQ models have also been widely studied in the control-theory literature for the special case when there are no forward-looking variables (see Costa and Fragoso [10], Costa, Fragoso, and Marques [11] (henceforth CFM), do Val, Geromel, and Costa [14], and the references therein). More recently, Zampolli [41] uses an MJLQ model to examine monetary policy under shifts between regimes with and without an asset-market bubble, although still in a model without forward-looking variables. Blake and Zampolli [4] provide an extension of the MJLQ model to include forward-looking variables, although with less generality than in our paper and with the analysis and the algorithms restricted to discretion equilibria.

Relative to this previous literature, our contribution is the development of a general approach for handling MJLQ models that include forward-looking variables. This extension is key for policy analysis under rational expectations, but the forward-looking variables make the model nonrecursive. We show that the recursive saddlepoint method of Marcet and Marimon [26] can nevertheless be applied to express the model in a convenient recursive way, and we derive an algorithm for determining the optimal policy and value functions.

In addition to considering the optimal policy, we also consider the behavior of the model for arbitrary time-varying or time-invariant instrument rules. This allows us to construct modelconsistent probability distributions -fan charts - of the variables relevant to policy makers for any arbitrary instrument-rate path. Moreover, much of the literature in monetary policy analysis has focused on "simple" instrument rules which are restricted to respond to only a subset of all available information, with Taylor rules and various generalizations being most prominent. We show how to derive optimal restricted instrument rules in our setting. Importantly, our approach is not restricted to instrument rules; any given or optimal restricted policy rule, including targeting rules, can be considered.

For most of the paper, we focus on the case where agents can directly observe the mode. While

In this [risk management] approach, a central bank needs to consider not only the most likely [rather: mean] future path for the economy but also the distribution of possible outcomes about that path. The decisionmakers then need to reach a judgment about the probabilities, costs, and benefits of various possible outcomes under alternative choices for policy.

We agree with Feldstein [15] that Greenspan's risk-management approach is best interpreted as standard expectedloss minimization and we consider the risk-management approach and the approach of this paper as completely consistent. See Blinder and Reis [5] for further discussion of possible interpretations of the risk-management approach. 
this may be plausible for some environments, such as for example when a new policy regime is announced, in many cases it is more fitting to assume that the modes are not observable. When the modes are not observable, we can represent the decision maker's information as a probability distribution over possible modes, and optimal policy will depend on that distribution. In this paper, we analyze the special case where decision makers do not learn from endogenous variables, but rather the future the subjective distribution over modes is entirely governed by the transition probabilities. In this case, the value function remains quadratic in the state, but with weights that depend now on the probability distribution over modes. We develop a simple method of solving this case.

The more general case where decision makers infer from their observations the probability of being in a particular mode is much more difficult to solve. The optimal filter is nonlinear, which destroys the tractability of the MJLQ approach. ${ }^{2}$ Additionally, as in most Bayesian learning problems, the optimal policy will also include an experimentation component. Thus, solving for the optimal decision rules will be a more complex numerical task. Due to the curse of dimensionality, it is only feasible in models with a relatively small number of state variables and modes. Confronted with these difficulties, the literature has focused on approximations such as linearization or adaptive control. ${ }^{3}$ While these issues are important, they remain outside the scope of the present paper.

The rest of the paper is organized as follows. In section 2, we lay out the model. In section 3, we discuss how different kinds of model uncertainty can be incorporated by our framework. In section 4, we present examples based on two empirical models of the US economy: regimeswitching versions of the backward-looking model of Rudebusch and Svensson [30] and the forwardlooking New Keynesian model of Lindé [24]. In section 5, we show how probability distributions of forecasts of relevant variables can be constructed for arbitrary time-varying instrument-rate paths or functions. In section 6 , we show how the same probability distributions can be constructed for arbitrary time-invariant instrument rules and optimal restricted instrument rules. Here we derive optimal generalized and mode-dependent Taylor-type rules in the Lindé model. In section 7, we show how the optimal policy and value functions can be expressed as a function of the probability distribution of the modes, in the realistic case when these modes are not observable.

\footnotetext{
${ }^{2}$ The optimal nonlinear filter is well-known, and it is a key component of the estimation methods as well (Hamilton [19] and Kim and Nelson [22]).

3 In the first case, restricting attention to (sub-optimal) linear filters preserves the tractability of the linearquadratic framework. See CFM [11] for a brief discussion and references. In adaptive control, agents do not take into account the informational role of their decisions. See do Val and Başar [13] for an application of an adaptive control MJLQ problem in economics. In a different setting, Cogley, Colacito, and Sargent [9] have recently studied how well adaptive procedures approximate the optimal policies.
} 
We then reconsider the examples from section 4 in the unobservable case, and find that the effects of unobservability differ greatly across the two models. In section 8 , we present some conclusions. The appendices contain some technical details and extensions of the material in the text.

\section{The model}

We set up a relatively flexible model of an economy with a central bank, which allows for relatively broad additive and multiplicative uncertainty as well as different relevant representations of the central-bank information and judgment about the economy.

\subsection{The baseline model}

Consider the following model of an economy with a central bank:

$$
\begin{aligned}
X_{t+1} & =A_{11, t+1} X_{t}+A_{12, t+1} x_{t}+B_{1, t+1} i_{t}+C_{t+1} \varepsilon_{t+1}, \\
\mathrm{E}_{t} H_{t+1} x_{t+1} & =A_{21, t} X_{t}+A_{22, t} x_{t}+B_{2, t} i_{t},
\end{aligned}
$$

where $X_{t}$ is an $n_{X} \times 1$ vector of predetermined variables (the state) in period $t$ (the first element may be unity to incorporate nonzero intercepts in a convenient way), $x_{t}$ is an $n_{x} \times 1$ vector of forwardlooking variables in period $t, i_{t}$ is an $n_{i} \times 1$ vector of central-bank instruments (control variables) in period $t$, and $\varepsilon_{t}$ is an $n_{X} \times 1$ vector of zero-mean i.i.d. shocks realized in period $t$ with covariance matrix $I$. The forward-looking variables and the instruments are the nonpredetermined variables. ${ }^{4}$ The matrix $A_{22, t}$ is nonsingular, so equation (2.2) determines the forward-looking variables in period $t$. There is no restriction in including the shock $\varepsilon_{t}$ only in the equations for the predetermined variables, since, if necessary, the set of predetermined variables can always be expanded to include the shocks and the shocks this way indirectly enter into the equations for the forward-looking variables. The expression $\mathrm{E}_{t} q_{t+1}$ denotes the conditional expectation in period $t$ of any random variable $q_{t+1}$ realized in period $t+1$. The informational assumptions underlying the conditional expectations operator $\mathrm{E}_{t}$ are specified below.

The central bank has an intertemporal loss function in period $t$ :

$$
\mathrm{E}_{t} \sum_{\tau=0}^{\infty} \delta^{\tau} L_{t+\tau},
$$

\footnotetext{
${ }^{4}$ Predetermined variables have exogenous one-period-ahead forecast errors, whereas non-predetermined variables have endogenous one-period-ahead forecast errors.
} 
where the period loss, $L_{t}$, satisfies

$$
L_{t} \equiv Y_{t}^{\prime} \Lambda_{t} Y_{t}
$$

where

$$
Y_{t} \equiv D_{t}\left[\begin{array}{c}
X_{t} \\
x_{t} \\
i_{t}
\end{array}\right]
$$

is an $n_{Y} \times 1$ vector of target variables and the weight matrix $\Lambda_{t}$ is a symmetric and positive semidefinite matrix. It follows that the period loss function satisfies

$$
L_{t} \equiv\left[\begin{array}{c}
X_{t} \\
x_{t} \\
i_{t}
\end{array}\right]^{\prime} W_{t}\left[\begin{array}{c}
X_{t} \\
x_{t} \\
i_{t}
\end{array}\right],
$$

where the matrix $W_{t} \equiv D_{t}^{\prime} \Lambda_{t} D_{t}$ is symmetric and positive semidefinite. The scalar $\delta$ is a discount factor satisfying $0<\delta \leq 1 .^{5}$

The matrices $A_{11, t}, A_{12, t}, B_{1, t}, C_{t}, H_{t}, A_{21, t}, A_{22, t}, B_{2, t}, \Lambda_{t}, D_{t}$, and $W_{t}$ (assumed to be of appropriate dimension) are random and can each take $n$ different values in period $t$, corresponding to the $n$ modes $j_{t}=1,2, \ldots, n$ in period $t$. We denote these values $A_{11, t}=A_{11 j_{t}}, A_{12, t}=A_{12 j_{t}}$, and so forth. The modes $j_{t}$ follow a Markov process with constant transition probabilities:

$$
P_{j k} \equiv \operatorname{Pr}\left\{j_{t+1}=k \mid j_{t}=j\right\} \quad(j, k=1, \ldots, n)
$$

While we focus throughout on the time-homogeneous case, it is straightforward to allow the modes to depend directly on calendar time. Furthermore, $P$ denotes the $n \times n$ transition matrix $\left[P_{j k}\right]$ and the $1 \times n$ vector $p \equiv\left(p_{1 t}, \ldots, p_{n t}\right)$ (where $\left.p_{j t} \equiv \operatorname{Pr}\left\{j_{t}=j\right\}, j=1, \ldots, n\right)$ denotes the probability distribution of the modes in period $t$, so

$$
p_{t+1}=p_{t} P
$$

Finally, the $1 \times n$ vector $\bar{p}$ denotes the unique stationary distribution of the modes, so ${ }^{6}$

$$
\bar{p}=\bar{p} P .
$$

The shocks $\varepsilon_{t}$ and the modes $j_{t}$ are assumed to be independently distributed (although we allow the impact on the economy of the shocks $\varepsilon_{t}$ to depend on the modes $j_{t}$ through the matrix

\footnotetext{
${ }^{5}$ When $\delta=1$, the loss function (2.3) normally becomes unbounded. To handle this case, we scale the intertemporal loss function by $1-\delta$ for $\delta<1$ and consider the loss function to be the limit $\lim _{\delta \rightarrow 1}(1-\delta) \mathrm{E}_{t} \sum_{\tau=0}^{\infty} \delta^{\tau} L_{t+\tau}$. See appendix C for details.

${ }^{6}$ We assume that the Markov chain is recurrent and aperiodic, so the stationary distribution is unique and does not depend on the initial mode (Karlin and Taylor [21]). A simple sufficient condition is that the matrix $P^{m}$ has all elements positive for some $m \geq 1$ (Ljungqvist and Sargent [25]).
} 
$C_{j_{t}}$ ). However, this assumption is not restrictive. Mode-dependent additive shocks are actually incorporated, since the fact that we allow one of the predetermined variables to be unity implies that all our equations may have mode-dependent intercepts. ${ }^{7}$

In the beginning of period $t$, before the central bank chooses the instruments, $i_{t}$, the central bank's information set includes the history of the realizations of $X_{t}, j_{t}, \varepsilon_{t}, X_{t-1}, j_{t-1}, \varepsilon_{t-1}, x_{t-1}$, $i_{t-1}, \ldots$ The central bank also knows the probability distribution of the innovation $\varepsilon_{t}$, the transition matrix $P$, and the $n$ different values each of the matrices can take. Hence, the conditional expectations operator, $\mathrm{E}_{t}$, refers to expectations conditional on that information. In section 7 we consider an alternative situation, which in many cases is more realistic, where the mode $j_{t}$ is not observable in period $t$, and hence policy in period $t$ is based on the probability distribution $p_{t}$ of the modes.

We consider the optimization problem of minimizing (2.3) in period $t$, subject to (2.1), (2.2), (2.4), and $X_{t}$ and $j_{t}$ given. In particular, we consider the optimization under commitment in a timeless perspective (see Woodford [40] and Svensson and Woodford [39]).

\subsection{Reformulation according to the recursive saddlepoint method}

In order to apply the methods developed for similar models in control theory, we require that the system be recursive. However, the presence of the forward-looking variables in (2.2) makes the problem nonrecursive. Fortunately, the recursive saddlepoint method of Marcet and Marimon [26] can be applied to reformulate the non-recursive problem with forward-looking variables as a recursive saddlepoint problem (see Marcet and Marimon [26] for the general method and Svensson [37] for the method applied to linear-quadratic problems).

More precisely, the problem of minimizing the intertemporal loss function in each period $t$ under commitment in a timeless perspective can be reformulated as the dual saddlepoint problem:

$$
\max _{\left\{\gamma_{t+\tau}\right\}_{\tau \geq 0}} \min _{\left\{x_{t+\tau}, i_{t+\tau}\right\}_{\tau \geq 0}} \mathrm{E}_{t} \sum_{\tau=0}^{\infty} \delta^{\tau} \tilde{L}_{t+\tau},
$$

with the dual period loss function,

$$
\tilde{L}_{t+\tau} \equiv\left[\begin{array}{c}
\tilde{X}_{t+\tau} \\
\tilde{\imath}_{t+\tau}
\end{array}\right]^{\prime} \tilde{W}_{j_{t+\tau}}\left[\begin{array}{c}
\tilde{X}_{t+\tau} \\
\tilde{\imath}_{t+\tau}
\end{array}\right]
$$

\footnotetext{
${ }^{7}$ Without significant loss of generality, we could assume that the $\varepsilon$ shocks are discrete, $\varepsilon_{t} \in\left\{\bar{\varepsilon}_{h}\right\}_{h=1}^{\bar{n}}$, and hence depend on separate modes $h=1, \ldots, \bar{n}$ which may be correlated with the $j$ modes. Then we could consider $n \bar{n}$ generalized modes $(j, h)(j=1, \ldots, n, h=1, \ldots, \bar{n})$ and incorporate the $\varepsilon$ shocks in intercepts that depend on the generalized modes. This way we could, without loss of generality, write the model without any explicit additive $\varepsilon$ shocks.
} 
subject to the dual model:

$$
\tilde{X}_{t+\tau+1}=\tilde{A}_{j_{t+\tau+1}} \tilde{X}_{t+\tau}+\tilde{B}_{j_{t+\tau+1}} \tilde{t}_{t+\tau}+\tilde{C}_{j_{t+\tau+1}} \varepsilon_{t+\tau+1}
$$

for $\tau \geq 0$, where $\tilde{X}_{t}$ and $j_{t}$ are given. Here, the new $n_{\tilde{X}} \times 1$ vector of predetermined variables $\tilde{X}_{t}$ $\left(n_{\tilde{X}} \equiv n_{X}+n_{x}\right)$ and the new $n_{\tilde{\imath}} \times 1$ vector of instruments $\tilde{\imath}_{t}\left(n_{\tilde{\imath}} \equiv n_{x}+n_{i}+n_{x}\right)$ are defined as

$$
\tilde{X}_{t} \equiv\left[\begin{array}{c}
X_{t} \\
\Xi_{t-1}
\end{array}\right], \quad \tilde{\imath}_{t} \equiv\left[\begin{array}{c}
x_{t} \\
i_{t} \\
\gamma_{t}
\end{array}\right]
$$

The elements of the $n_{x} \times 1$ vector $\Xi_{t-1}$ are the Lagrange multipliers for the equations (2.2) for the forward-looking variables in period $t-1$ from the optimization problem in that period. Hence, $\Xi_{t-1}$ captures the history dependence of the optimal policy under commitment in a timeless perspective (see Woodford [40] and Svensson and Woodford [39]). The elements of the $n_{x} \times 1$ vector $\gamma_{t}$ are the Lagrange multipliers for equations (2.2) in period $t$, considered as control variables in period $t$. Hence, we have

$$
\Xi_{t}=\gamma_{t}
$$

as an additional dynamic equation, which is incorporated in (2.8).

The matrix $\tilde{W}_{j_{t}}$ in $(2.7)$ is constructed so the dual period loss $\tilde{L}_{t}$ satisfies

$$
\tilde{L}_{t} \equiv L_{t}-\gamma_{t}^{\prime}\left(A_{21 j_{t}} X_{t}+A_{22 j_{t}} x_{t}+B_{2 j_{t}} i_{t}\right)+\frac{1}{\delta} \Xi_{t-1}^{\prime} H_{j_{t}} x_{t}
$$

The matrices $\tilde{A}_{j_{t+1}}, \tilde{B}_{j_{t+1}}$, and $\tilde{C}_{j_{t+1}}$ (partitioned conformably with $\tilde{X}_{t}$ and, for $\tilde{B}_{j_{t+1}}$, also with $\tilde{\imath}_{t}$ ) satisfy

$$
\tilde{A}_{j_{t+1}} \equiv\left[\begin{array}{cc}
A_{11 j_{t+1}} & 0 \\
0 & 0
\end{array}\right], \quad \tilde{B}_{j_{t+1}} \equiv\left[\begin{array}{ccc}
A_{12 j_{t+1}} & B_{1 j_{t+1}} & 0 \\
0 & 0 & I
\end{array}\right], \quad \tilde{C}_{j_{t}} \equiv\left[\begin{array}{c}
C_{j_{t+1}} \\
0
\end{array}\right] .
$$

\subsection{Optimal policy and dynamics}

The solution of the dual saddlepoint problem will result in a conditionally linear optimal policy function with mode-dependent coefficients,

$$
\tilde{\imath}_{t}=F_{j_{t}} \tilde{X}_{t} \quad\left(j_{t}=1, \ldots, n\right)
$$

and a dual conditionally quadratic value function with mode-dependent coefficients,

$$
\tilde{X}_{t} \tilde{V}_{j_{t}} \tilde{X}_{t}+\tilde{w}_{j_{t}}=\max _{\left\{\gamma_{t+\tau}\right\}_{\tau \geq 0}} \min _{\left\{x_{t+\tau}, i_{t+\tau}\right\}_{\tau \geq 0}} \mathrm{E}_{t} \sum_{\tau=0}^{\infty} \delta^{\tau} \tilde{L}_{t+\tau}, \quad\left(j_{t}=1, \ldots, n\right)
$$


(see appendix B for details and a convenient algorithm for computing $\tilde{V}_{j}$ and $F_{j}$ for $j=1, \ldots, n$ ). The optimal policy function for the dual problem is also the solution to the original problem.

Consider the composite state $\left(\tilde{X}_{t}, j_{t}\right)$ in period $t$, where $\tilde{\imath}_{t}=F_{j_{t}} \tilde{X}_{t}$. The transition from this composite state to the composite state $\left(\tilde{X}_{t+1}, j_{t+1}\right)$ in period $t+1$ with $\tilde{\imath}_{t+1}=F_{j_{t+1}} \tilde{X}_{t+1}$ will satisfy

$$
\tilde{X}_{t+1}=M_{j_{t} j_{t+1}} \tilde{X}_{t}+\tilde{C}_{j_{t+1}} \varepsilon_{t+1}
$$

where

$$
M_{j_{t} j_{t+1}} \equiv \tilde{A}_{j_{t+1}}+\tilde{B}_{j_{t+1}} F_{j_{t}}
$$

and will, for given realization of $\varepsilon_{t+1}$, occur with probability $P_{j_{t} j_{t+1}}$. This determines the optimal distribution of future $\tilde{X}_{t+\tau}, j_{t+\tau}$, and $\tilde{\imath}_{t+\tau}(\tau \geq 1)$ conditional on $\left(\tilde{X}_{t}, j_{t}\right)$.

Such conditional distributions can be illustrated by plots of future means, medians, and percentiles (fan charts). Plots of future means, medians, and percentiles can also be constructed for individual chains of the modes, for instance, the median or mean chain corresponding to no model uncertainty. The simplest way to generate such plots is by simulation, which we illustrate in some examples below.

Note that the value function in (2.14) above corresponds to the dual period loss function and the dual saddlepoint problem. The value function for the original problem of minimizing (2.3) subject to $(2.1),(2.2)$, and (2.4) under commitment in a timeless perspective with $\tilde{X}_{t}$ given is

$$
\tilde{X}_{t}^{\prime} V_{j_{t}} \tilde{X}_{t}+w_{j_{t}}
$$

The matrices $V_{j}$ and the scalars $w_{j}$ for $j=1, \ldots, n$, can be determined in the following way.

Let $F_{j_{t}}$ be partitioned conformably with $x_{t}, i_{t}$, and $\gamma_{t}$,

$$
F_{j_{t}} \equiv\left[\begin{array}{c}
F_{x j_{t}} \\
F_{i j_{t}} \\
F_{\gamma j_{t}}
\end{array}\right] \text {, }
$$

and note that we have

$$
\left[\begin{array}{c}
X_{t} \\
x_{t} \\
i_{t}
\end{array}\right]=\left[\begin{array}{cc}
I & 0 \\
F_{x j_{t}} \\
F_{i j_{t}}
\end{array}\right] \tilde{X}_{t} .
$$

It follows that we can write the period loss function as

$$
L_{t}=\tilde{X}_{t}^{\prime} \bar{W}_{j_{t}} \tilde{X}_{t}
$$

where

$$
\bar{W}_{j_{t}} \equiv\left[\begin{array}{cc}
I & 0 \\
F_{x j_{t}} \\
F_{i j_{t}}
\end{array}\right]^{\prime} W_{j_{t}}\left[\begin{array}{cc}
I & 0 \\
F_{x j_{t}} \\
F_{i j_{t}}
\end{array}\right]
$$


For each $j=1, \ldots, n$, the matrix $V_{j}$ will then satisfy the Lyapunov equation:

$$
V_{j}=\bar{W}_{j}+\delta \sum_{k} P_{j k} M_{j k}^{\prime} V_{k} M_{j k}
$$

and the scalar $w_{j}$ will satisfy the equation: ${ }^{8}$

$$
w_{j}=\delta \sum_{k} P_{j k}\left[\operatorname{tr}\left(V_{k} \tilde{C}_{k} \tilde{C}_{k}^{\prime}\right)+w_{k}\right]
$$

The matrices $V_{j}$ and the scalar $w_{j}$ can also be found in a more direct way from the matrices $\tilde{V}_{j}$ and the scalar $\tilde{w}_{j}$. Note that, by $(2.2),(2.11)$, and (2.10), the identity

$$
\tilde{X}_{t}^{\prime} V_{j} \tilde{X}_{t}+w_{j} \equiv \tilde{X}_{t}^{\prime} \tilde{V}_{j} \tilde{X}_{t}+\tilde{w}_{j}-\frac{1}{\delta} \Xi_{t-1}^{\prime} H_{j} F_{x j} \tilde{X}_{t} \quad(j=1, \ldots, n)
$$

must hold. We can write

$\frac{1}{\delta} \Xi_{t-1}^{\prime} H_{j} F_{x j} \tilde{X}_{t} \equiv \frac{1}{2 \delta}\left(\Xi_{t-1}^{\prime} H_{j} F_{x j} \tilde{X}_{t}+\tilde{X}_{t}^{\prime} F_{x j}^{\prime} H_{j}^{\prime} \Xi_{t-1}\right) \equiv \tilde{X}_{t}^{\prime}\left[\begin{array}{cc}0 & \frac{1}{2 \delta} F_{x X j}^{\prime} H_{j}^{\prime} \\ \frac{1}{2 \delta} H_{j} F_{x X j} & \frac{1}{2 \delta}\left(H_{j} F_{x \Xi j}+F_{x \Xi j}^{\prime} H_{j}^{\prime}\right)\end{array}\right] \tilde{X}_{t}$,

where $F_{x j}$ is partitioned conformably with $X_{t}$ and $\Xi_{t-1}$ as $F_{x j} \equiv\left[\begin{array}{ll}F_{x X j} & F_{x \Xi j}\end{array}\right]$. Then, identification of terms implies that $w_{j}$ and $V_{j}$ are determined by

$$
\begin{gathered}
w_{j}=\tilde{w}_{j} \quad(j=1, \ldots, n), \\
V_{j}=\tilde{V}_{j}-\left[\begin{array}{cc}
0 & \frac{1}{2 \delta} F_{x X j}^{\prime} H_{j}^{\prime} \\
\frac{1}{2 \delta} H_{j} F_{x X j} & \frac{1}{2 \delta}\left(H_{j} F_{x \Xi j}+F_{x \Xi j}^{\prime} H_{j}^{\prime}\right)
\end{array}\right] \quad(j=1, \ldots, n) .
\end{gathered}
$$

As discussed in CFM [11], mean square stability is an appropriate concept of stability for our framework. Appendix D provides some details on the definition of mean square stability and shows how the necessary and sufficient conditions for mean square stability derived in CFM [11] can be applied to our framework.

\section{Interpretation of model uncertainty in our framework}

The assumption that the random matrices of coefficients take a finite number of values corresponding to a finite number of modes allows us to use the convenient and flexible framework of MJLQ systems - once we apply the recursive saddlepoint method of Marcet and Marimon to reformulate the non-recursive model with forward-looking variables as a recursive model. By specifying different configurations of modes and transition probabilities, we can approximate many different kinds of model uncertainty.

\footnotetext{
${ }^{8}$ Note that $\tilde{C}_{k} \tilde{C}_{k}^{\prime}$ is the covariance matrix of the shocks $\tilde{C}_{k} \varepsilon_{t+1}$ to $\tilde{X}_{t+1}$ when $j_{t+1}=k(k=1, \ldots, n)$. Note also that $w_{j}$ will normally have a bounded solution only if $\delta<1$. See appendix $\mathrm{C}$ for how to handle the case $\delta=1$.
} 
- Both i.i.d. and serially correlated random coefficients of the model can be handled. This can capture either generalized parameter uncertainty or different behavior in different modeled regimes (such boom/bust states, and so forth).

- The modes can correspond to different structural models. The models can differ by having different relevant variables, different number of leads or lags, or the same variable being predetermined in one model and forward-looking in another. For example, one mode can represent a model with forward-looking variables such as the New Keynesian model of Lindé [24], another a backward-looking model such as that of Rudebusch and Svensson [30] (see appendix E for details).

- The modes can correspond to situations when variables such as inflation and output have more or less inherent persistence (are more or less autocorrelated), when the exogenous shocks have more or less persistence (introduce a predetermined variable equal to the serially correlated shock, letting it be an $\mathrm{AR}(1)$ process with a high or low coefficient), or when the uncertainty about the coefficients or models is higher or lower.

- The modes can be structured such that they correspond to different central-bank judgments about model coefficients and model uncertainty. Let $j_{t}=1, \ldots, n$ correspond to $n$ different model modes (different coefficients, different variances or persistence of coefficient disturbances, or different variances of the $\varepsilon_{t}$ shocks). Let $k_{t}=1, \ldots, m$ correspond to $m$ different central-bank judgment modes, depicting some central-bank information about the model modes. This can generally be modeled as a situation where the transition matrix for the model modes depends on the judgment mode. Thus let the transition matrix for model modes be $\tilde{P}\left(k_{t}\right)$, and hence depend on $k_{t}$. Let $P^{0}$ denote the transition matrix for the judgment modes (assumed independent of the model modes). We can then consider a composite modeljudgment mode $\left(j_{t}, k_{t}\right)$ in period $t$, with the transition probability from model-judgment mode $(h, k)$ in period $t$ to mode $(j, l)$ in period $t+1$ given by $\tilde{P}(k)_{h j} P_{k l}^{0}$. For instance, the judgment modes may correspond to different persistence of the model modes.

- The mode $j_{t}$ may be observed in period $t$, in which case optimal policy and the value function is conditional on the mode $j_{t}$, as shown above. Alternatively, and more realistically, we may assume that the mode cannot be perfectly observed. Then we can represent the central bank's information in period $t$ about the mode as the distribution $p_{t}$ of the modes. Then optimal 
policy and the value function in period $t$ will depend on the distribution $p_{t}$. This case is considered in section 7 .

- As noted in appendix A, we can combine multiplicative uncertainty about the modes with the additive uncertainty about future deviations. This way we can simultaneously handle central-bank judgment about future additive deviations as in Svensson [36] and central-bank judgment about model modes as in this paper. For instance, we can handle situations when there is more or less uncertainty about shocks farther into the future relative to those in the near future.

Generally, aside from dimensional and computational limitations, it is difficult to conceive of a situation for a policymaker that cannot be approximated in this framework.

\section{Examples}

In this section we present examples based on two empirical models of the US economy: regimeswitching versions of the backward-looking model of Rudebusch and Svensson [30] and the forwardlooking New Keynesian model of Lindé [24].

\subsection{An estimated backward-looking model}

In this section we consider the effects of model uncertainty in the quarterly model of the US economy of Rudebusch and Svensson [30], henceforth RS. Using the same data set as in their paper, we estimate a three-mode MJLQ model using Bayesian methods to locate the maximum of the posterior distribution, and we compare the implications to the constant-coefficient version of RS.

The key variables in the model are quarterly annualized inflation $\pi_{t}$, the output gap $y_{t}$, and the instrument rate (the federal funds rate) $i_{t}$. The model has a Phillips curve and an aggregate-demand relation of the following form:

$$
\begin{aligned}
\pi_{t+1} & =\sum_{\tau=0}^{2} \alpha_{\tau j} \pi_{t-\tau}+\left(1-\sum_{\tau=0}^{2} \alpha_{\tau j}\right) \pi_{t-3}+\alpha_{3 j} y_{t}+c_{\pi j} \varepsilon_{\pi, t+1}, \\
y_{t+1} & =\beta_{1 j} y_{t}+\beta_{2 j} y_{t-1}+\beta_{3 j}\left(\bar{\imath}_{t}-\bar{\pi}_{t}\right)+c_{y i} \varepsilon_{y, t+1},
\end{aligned}
$$

where $j \in\{1,2,3\}$ indexes the mode, $\bar{\imath}_{t} \equiv \sum_{\tau=0}^{3} i_{t-\tau} / 4$ and $\bar{\pi}_{t} \equiv \sum_{\tau=0}^{3} \pi_{t-\tau} / 4$ are 4-quarter averages, and the shocks $\varepsilon_{\pi t}$ and $\varepsilon_{y t}$ are each independent standard normal random variables. 


\begin{tabular}{c|r|rrr}
\hline \hline Parameter & Constant & Mode 1 & Mode 2 & Mode 3 \\
\hline$\alpha_{0}$ & 0.6922 & 0.2402 & 0.4236 & 1.2387 \\
$\alpha_{1}$ & -0.1033 & 0.1654 & -0.2219 & -0.6911 \\
$\alpha_{2}$ & 0.2786 & 1.0388 & 0.0714 & 0.5491 \\
$\alpha_{3}$ & 0.1284 & 0.1514 & 0.2755 & -0.0304 \\
$\beta_{1}$ & 1.1591 & 1.0015 & 1.0302 & 1.8943 \\
$\beta_{2}$ & -0.2521 & -0.0853 & -0.1069 & -1.0312 \\
$\beta_{3}$ & -0.0990 & -0.3244 & 0.0315 & -0.1011 \\
$c_{\pi}$ & 0.9962 & 1.5504 & 0.1798 & 0.1562 \\
$c_{y}$ & 0.8132 & 1.2696 & 0.1447 & 0.2365 \\
\hline \hline
\end{tabular}

Table 4.1: Estimates of the constant-coefficient and three-mode Rudebusch-Svensson model.

Table 4.1 reports our estimates of the peak of the posterior, with the OLS estimates of the constant-coefficient version of the model for comparison. For the MJLQ model, we center our prior distribution at the OLS estimates. Details of the estimation method and prior setting are given in appendix F. Here we see that many of the coefficients differ substantially across modes. Perhaps most notable is the large difference in volatility, as the standard deviations of the shocks $\left(c_{\pi}\right.$ and $c_{y}$ ) are from five to ten times larger in mode 1 than in the other two modes. In addition, the slope of the Phillips curve, $\alpha_{3}$, ranges from a large positive response in mode 2 to a small negative value in mode 3. Similarly, the slope of the IS curve, $\beta_{3}$, ranges from a relatively large negative response in mode 1 to a small positive one in mode 2. The large differences in these key model coefficients lead to sharp differences in the optimal policy across modes, as we show below.

The estimated probabilities of being in the different modes are shown in figure 4.1. The plots show both the filtered estimates, in which the distribution in period $t$ is estimated using data only up to $t$, as well as the smoothed estimates, in which the distribution in period $t$ is estimated using data for the whole sample. Clearly, there are more fluctuations in the filtered estimates than in the smoothed ones, since by looking backward we can better assess the probability of being in a particular regime. We see that, for the early part of the sample, the economy was mostly assessed to be in the more volatile mode 1 . From the early 1980s onward, the modes 2 and 3 were more prominent, as the volatility moderated. The estimated transition matrix $P$ and its implied stationary distribution $\bar{p}$ are

$$
P=\left[\begin{array}{lll}
0.8331 & 0.0921 & 0.0748 \\
0.0305 & 0.9194 & 0.0501 \\
0.0360 & 0.0541 & 0.9100
\end{array}\right], \quad \bar{p}=\left[\begin{array}{lll}
0.1652 & 0.4483 & 0.3866
\end{array}\right]
$$

From the standpoint of these estimates, the early part of the sample is a bit of an aberration, as mode 1 has the lowest weight in the stationary distribution. Thus, although similar episodes will 

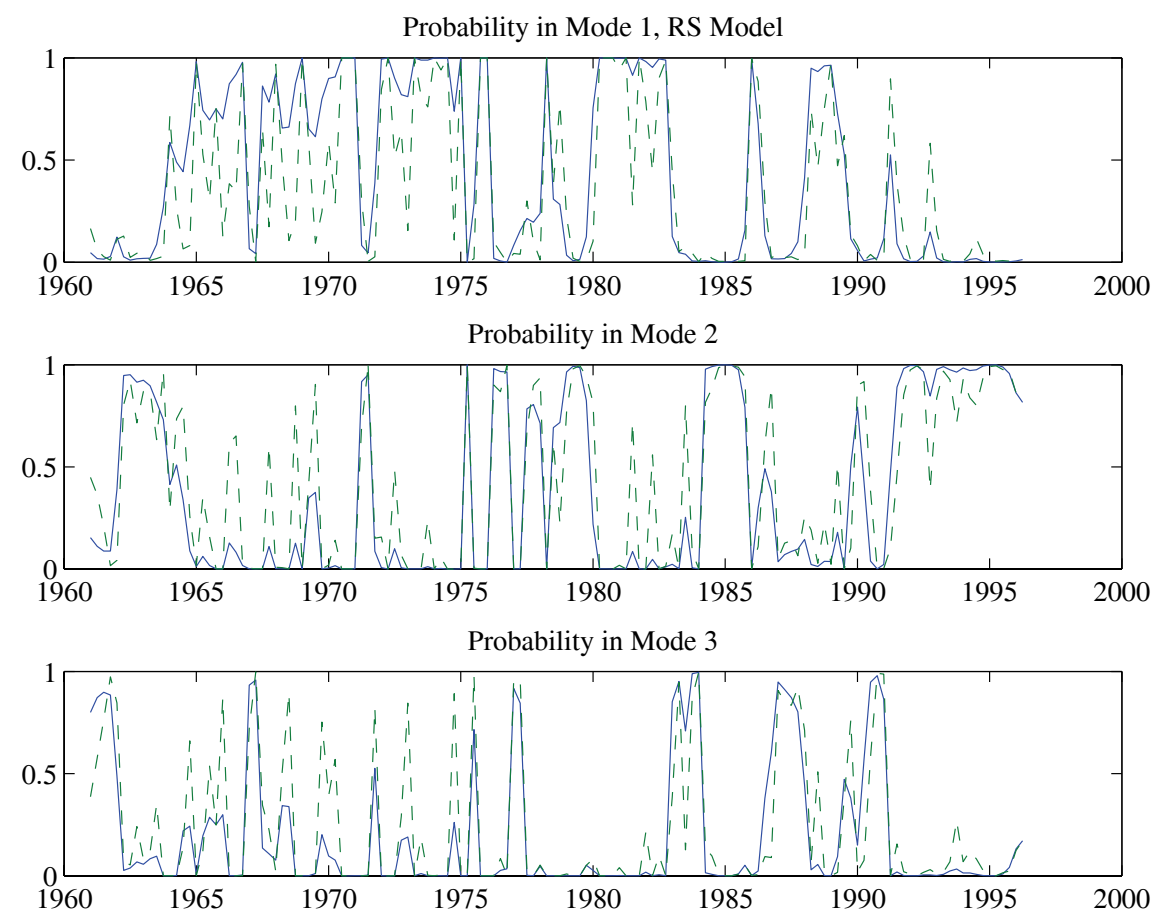

Figure 4.1: Estimated probabilities of being in the different modes. Smoothed (full-sample) inference is shown with solid lines, while filtered (one-sided) inference is shown with dashed lines.

re-occur in the model, they would tend to be balanced with longer periods of more tranquility.

We let the period loss function be

$$
L_{t}=\pi_{t}^{2}+\lambda y_{t}^{2}+\nu\left(i_{t}-i_{t-1}\right)^{2}
$$

Hence, the vector of target variables is $Y_{t} \equiv\left(\pi_{t}, y_{t}, i_{t}-i_{t-1}\right)^{\prime}$ and the weight matrix $\Lambda$ is a diagonal matrix with the diagonal $(1, \lambda, \nu)^{\prime}$. We set the weights to $\lambda=1$ and $\nu=0.2$. We set the discount factor in the intertemporal loss function to $\delta=1$. We then solve for the optimal policy function,

$$
i_{t}=F_{i j} X_{t}, \quad(j=1,2,3)
$$

where $X_{t} \equiv\left(\pi_{t}, \pi_{t-1}, \pi_{t-2}, \pi_{t-3}, y_{t}, y_{t-1}, i_{t-1}, i_{t-2}, i_{t-3}\right)^{\prime}$, using the methods described above.

The optimal policy functions are given in table 4.2. In figure 4.2, we plot the distribution of the impulse responses of inflation, the output gap, and the instrument rate to the two shocks in the model. In particular, for 10,000 simulation runs, we first draw an initial mode of the Markov chain from its stationary distribution, then simulate the chain for 50 periods forward, tracing out the impulse responses. The figure plots the mean response at each date, along with $30 \%$ quantiles of 
the empirical distribution. More precisely, the dark, medium, and light grey band show 30\%, $60 \%$, and $90 \%$ probability bands, respectively, with $5 \%$ of the distribution above the light gray band and $5 \%$ below. Also shown for comparison are the responses under the optimal policy for the estimated constant-coefficient model given above. ${ }^{9}$

\begin{tabular}{c|rrrrrrrrr}
\hline \hline Mode & $\pi_{t}$ & $\pi_{t-1}$ & $\pi_{t-2}$ & $\pi_{t-3}$ & $y_{t}$ & $y_{t-1}$ & $i_{t-1}$ & $i_{t-2}$ & $i_{t-3}$ \\
\hline Constant & 0.9921 & 0.3465 & 0.4273 & 0.1381 & 1.7974 & -0.4639 & 0.3713 & -0.0899 & -0.0456 \\
\hline Mode 1 & 1.4796 & 1.3130 & 1.0760 & -0.2853 & 1.9834 & -0.4890 & -0.1723 & -0.3271 & -0.1834 \\
Mode 2 & -0.1510 & -0.1739 & -0.2132 & -0.2077 & -1.0595 & -0.2824 & 0.3311 & -0.0840 & -0.0326 \\
Mode 3 & 1.1526 & 0.0988 & 0.5878 & 0.0309 & 4.6475 & -4.6851 & -0.0205 & -0.2364 & -0.1245 \\
\hline \hline
\end{tabular}

Table 4.2: Optimal policy functions for the constant-coefficient and three-mode RudebuschSvensson model.

Both the table and the figure illustrate that the model uncertainty leads to a change in the nature of policy. Compared to the constant-coefficient model, most of the mass of the distribution of the impulse responses lies closer to zero. This is particularly the case for the instrument-rate responses. Thus our results here are in accord with the common intuition based on Brainard [6], that model uncertainty should lead to less aggressive (that is, smaller in magnitude) policy responses. ${ }^{10}$ Interestingly, the probability distributions of responses are asymmetric, with the mean impulse responses different from the median responses (the latter lie inside the dark gray bands). In many cases, the tails of the distributions appear relatively wide. This is perhaps most noticeable in the responses of inflation to the two shocks. Here again the bulk of the distribution lies below the constant-coefficient model responses, but there is a significant right tail showing relatively large and persistent effects of the initial shock. These results illustrate that with model uncertainty policy makers must go beyond forecasting the means of target variables and consider the entire forecast distributions, and our approach makes this process quite manageable.

\subsection{An estimated forward-looking model}

We now consider the effects of uncertainty in a model with both forward- and backward-looking variables. The structural model is a mode-dependent simplification of the model of the US economy

\footnotetext{
${ }^{9}$ The shocks are $\varepsilon_{\pi 0}=1$ and $\varepsilon_{y 0}=1$, respectively, for the two different columns in the figure. Thus the shocks to inflation and the output gap in period 0 are mode dependent and equal to $c_{\pi j}$ and $c_{y j}(j=1,2,3)$, respectively. We initialize by drawing from the stationary distribution, so the distribution of modes in each period remains the stationary distribution.

${ }^{10}$ Of course, this is only a loose parallel, as the Brainard result need not apply for the type of uncertainty considered here, especially since the policy is here allowed to be mode-dependent.
} 

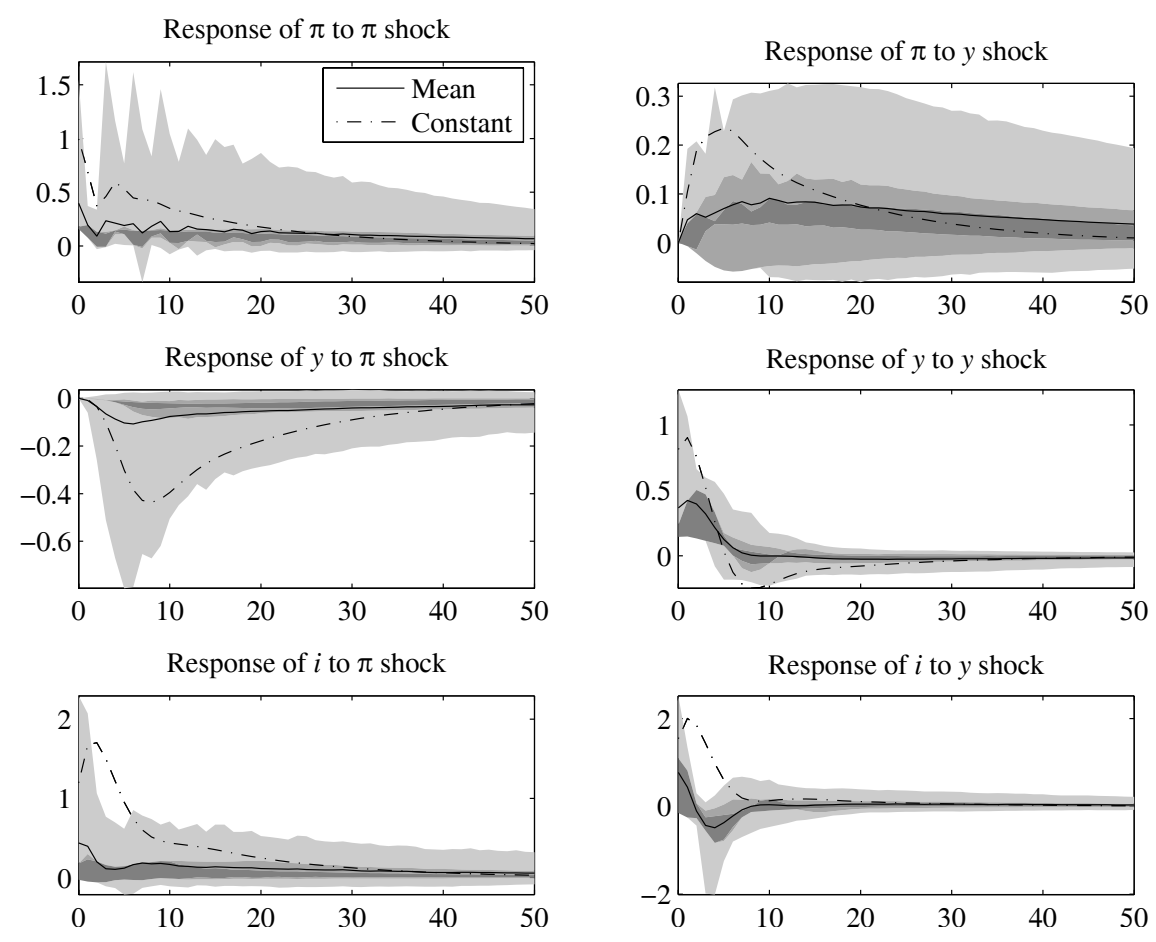

Figure 4.2: Unconditional impulse responses to shocks under the optimal policy for the modedependent Rudebusch-Svensson model. Solid lines: Mean responses. Dark/medium/light gray bands: 30/60/90\% probability bands. Dashed lines: Optimal responses for the constant-coefficient model.

of Lindé [24] and is given by

$$
\begin{aligned}
\pi_{t} & =\omega_{f j} E_{t} \pi_{t+1}+\left(1-\omega_{f j}\right) \pi_{t-1}+\gamma_{j} y_{t}+c_{\pi j} \varepsilon_{\pi t}, \\
y_{t} & =\beta_{f j} E_{t} y_{t+1}+\left(1-\beta_{f j}\right)\left[\beta_{y j} y_{t-1}+\left(1-\beta_{y j}\right) y_{t-2}\right]-\beta_{r j}\left(i_{t}-E_{t} \pi_{t+1}\right)+c_{y j} \varepsilon_{y t}, \\
i_{t} & =\left(1-\rho_{1 j}-\rho_{2 j}\right)\left(\gamma_{\pi j} \pi_{t}+\gamma_{y j} y_{t}\right)+\rho_{1 j} i_{t-1}+\rho_{2 j} i_{t-2}+c_{i j} \varepsilon_{i t},
\end{aligned}
$$

where an instrument rule is added to the Phillips curve and the aggregate-demand relation. ${ }^{11}$ Again, $j \in\{1,2,3\}$ indexes the mode, and the shocks $\varepsilon_{\pi t}, \varepsilon_{y t}$, and $\varepsilon_{i t}$ are independent standard normal random variables. We use the same data set as above, and again estimate a three-mode MJLQ model along with a constant-coefficient model using Bayesian methods. Once again, we explicitly state our prior settings in appendix F. We use the same prior for the structural coefficients in the constant-coefficient and MJLQ cases, and the priors for the Markov chain coefficients are the same

\footnotetext{
${ }^{11}$ Because of the forward-looking expectations in the model, estimation of the model requires that a policy rule be specified.
} 
as in the RS model.

\begin{tabular}{c|r|rrr}
\hline \hline Parameter & Constant & Mode 1 & Mode 2 & Mode 3 \\
\hline$\omega_{f}$ & 0.4908 & 0.4644 & 0.3380 & 0.3198 \\
$\gamma$ & 0.0081 & 0.0112 & 0.0786 & 0.0312 \\
$\beta_{f}$ & 0.4408 & 0.0889 & 0.2356 & 0.3911 \\
$\beta_{r}$ & 0.0048 & 0.0396 & 0.1395 & 0.0000 \\
$\beta_{y}$ & 1.1778 & 1.1119 & 1.1570 & 1.2312 \\
$\rho_{1}$ & 0.9557 & 1.1486 & 0.8525 & 0.7967 \\
$\rho_{2}$ & -0.0673 & -0.2340 & -0.1172 & 0.0516 \\
$\gamma_{\pi}$ & 1.3474 & 1.2439 & -0.0643 & 2.3427 \\
$\gamma_{y}$ & 0.7948 & 0.5799 & 0.9717 & -0.3101 \\
$c_{\pi}$ & 0.5923 & 0.4861 & 0.7232 & 0.9801 \\
$c_{y}$ & 0.4162 & 0.4744 & 0.5083 & 0.6720 \\
$c_{i}$ & 0.9918 & 0.2995 & 0.3930 & 1.2341 \\
\hline \hline
\end{tabular}

Table 4.3: Estimates of the constant-coefficient and three-mode Lindé model.

Table 4.3 reports our estimates, with the estimates from the constant-coefficient version of the model for comparison. Our constant-coefficient estimates are similar to those in Lindé [24], with the main difference that we find much smaller estimates of $\gamma$ and $\beta_{r}$. At least some of the difference may be due to our different data series and sample period. We again see that many of the key structural coefficients change substantially across modes, particularly the instrumentrule coefficients and shock standard deviations. For example, mode 3 has the largest shocks to all variables, while mode 1 has the smallest. The instrument-rule coefficients $\gamma_{\pi}$ and $\gamma_{y}$ in mode 1 are relatively close to those of the constant-coefficient model, while in mode 3 the response to inflation, $\gamma_{\pi}$, is actually negative.

The estimated transition matrix $P$ and its implied stationary distribution $\bar{p}$ are given by

$$
P=\left[\begin{array}{lll}
0.9403 & 0.0340 & 0.0257 \\
0.0625 & 0.8924 & 0.0451 \\
0.0695 & 0.0576 & 0.8729
\end{array}\right], \quad \bar{p}=\left[\begin{array}{lll}
0.5229 & 0.2741 & 0.2030
\end{array}\right]
$$

Thus mode 1 is the most persistent and has the largest mass in the invariant distribution. This is consistent with our estimation of the modes as shown in figure 4.3. Again, the plots show both the smoothed and filtered estimates. We see that mode 1 was experienced the most throughout much of the sample, holding for most of the sample until 1970 and then most of time after 1985. The volatile mode 3 held for much of the early 1970s and early 1980s, alternating with the intermediate mode 2 .

We again solve for the optimal policy function,

$$
i_{t}=F_{i j} \tilde{X}_{t}
$$



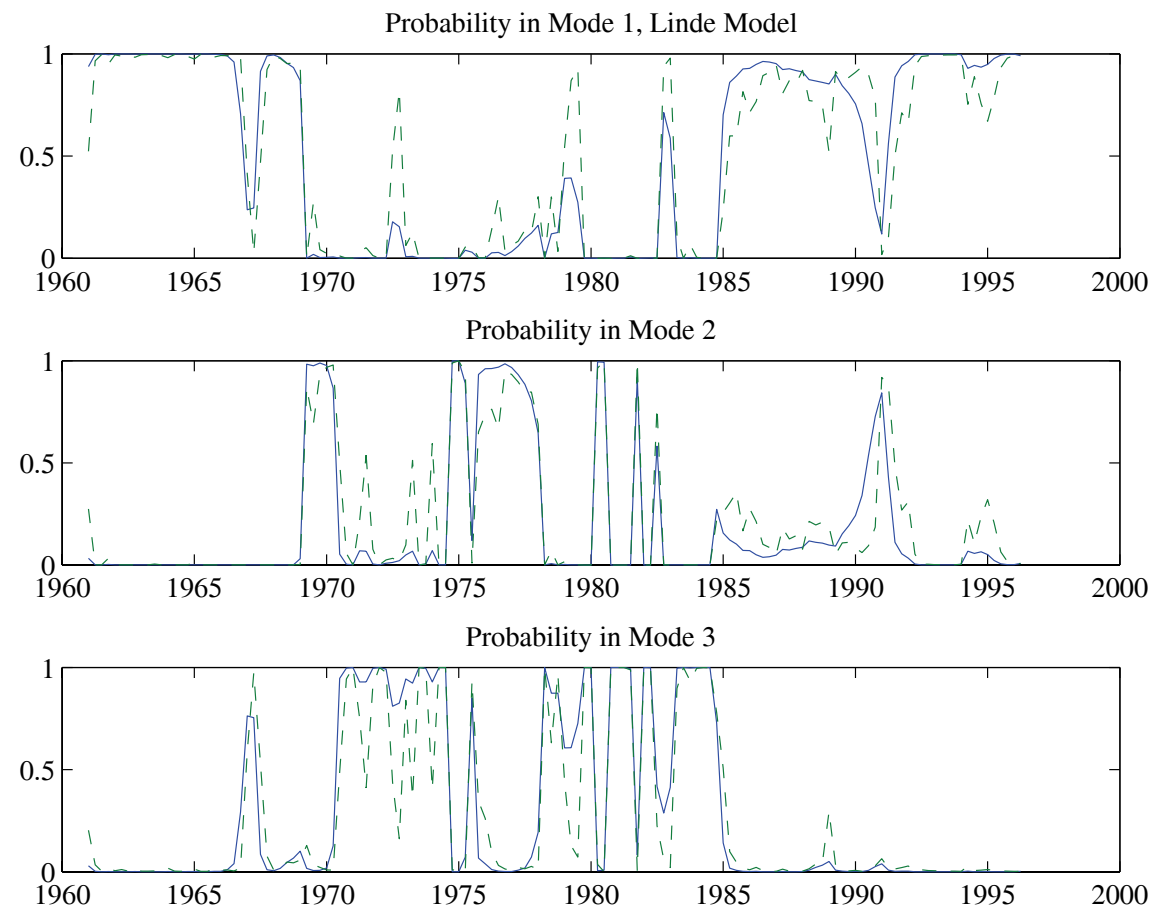

Figure 4.3: Estimated probabilities of being the different modes. Smoothed (full-sample) inference is shown with solid lines, while filtered (one-sided) inference is shown with dashed lines.

\begin{tabular}{c|rrrrrrrr}
\hline \hline Mode & $\pi_{t-1}$ & $y_{t-1}$ & $y_{t-2}$ & $i_{t-1}$ & $\varepsilon_{\pi t}$ & $\varepsilon_{y t}$ & $\Xi_{\pi, t-1}$ & $\Xi_{y, t-1}$ \\
\hline Constant & 0.3552 & 1.0714 & -0.2231 & 0.7853 & 0.6975 & 2.2437 & 0.0024 & 0.0182 \\
\hline Mode 1 & 0.8915 & 2.0766 & -0.2338 & 0.5962 & 1.6644 & 2.2929 & 0.0037 & 0.0066 \\
Mode 2 & 1.4625 & 1.6985 & -0.2666 & 0.3271 & 2.2092 & 2.2216 & 0.0090 & 0.0393 \\
Mode 3 & 0.8348 & 0.7955 & -0.2085 & 0.8016 & 1.2273 & 1.4812 & 0.0006 & 0.0021 \\
\hline \hline
\end{tabular}

Table 4.4: Optimal policy functions of the constant-coefficient and three-mode Lindé model.

where $\tilde{X}_{t} \equiv\left(\pi_{t-1}, y_{t-1}, y_{t-2}, i_{t-1}, \varepsilon_{\pi t}, \varepsilon_{y t}, \Xi_{\pi, t-1}, \Xi_{y, t-1}\right)^{\prime}$, using the methods described above. We use the same loss function as for the backward-looking model. The optimal policy functions are given in table 4.4. In figure 4.4, we plot the distribution of the impulse responses of inflation, the output gap, and the instrument rate to the two structural shocks in the model. Again we consider 10,000 simulations of 50 periods, and plot the mean responses along with $30 \%$ probability bands and the corresponding optimal responses for the constant-coefficient model. ${ }^{12}$

Again, the model uncertainty leads to a change in the nature of policy. Compared to the constant-coefficient case, most of the mass of the distribution of impulse responses is consistent

\footnotetext{
${ }^{12}$ Again, the shocks are $\varepsilon_{\pi 0}=1$ and $\varepsilon_{y 0}=1$, respectively, so the shocks to the inflation and output-gap equations in period 0 are mode-dependent and equal to $c_{\pi j}$ and $c_{y j}(j=1,2,3)$, respectively. The distribution of modes in period 0 (and thereby all periods) is again the stationary distribution.
} 

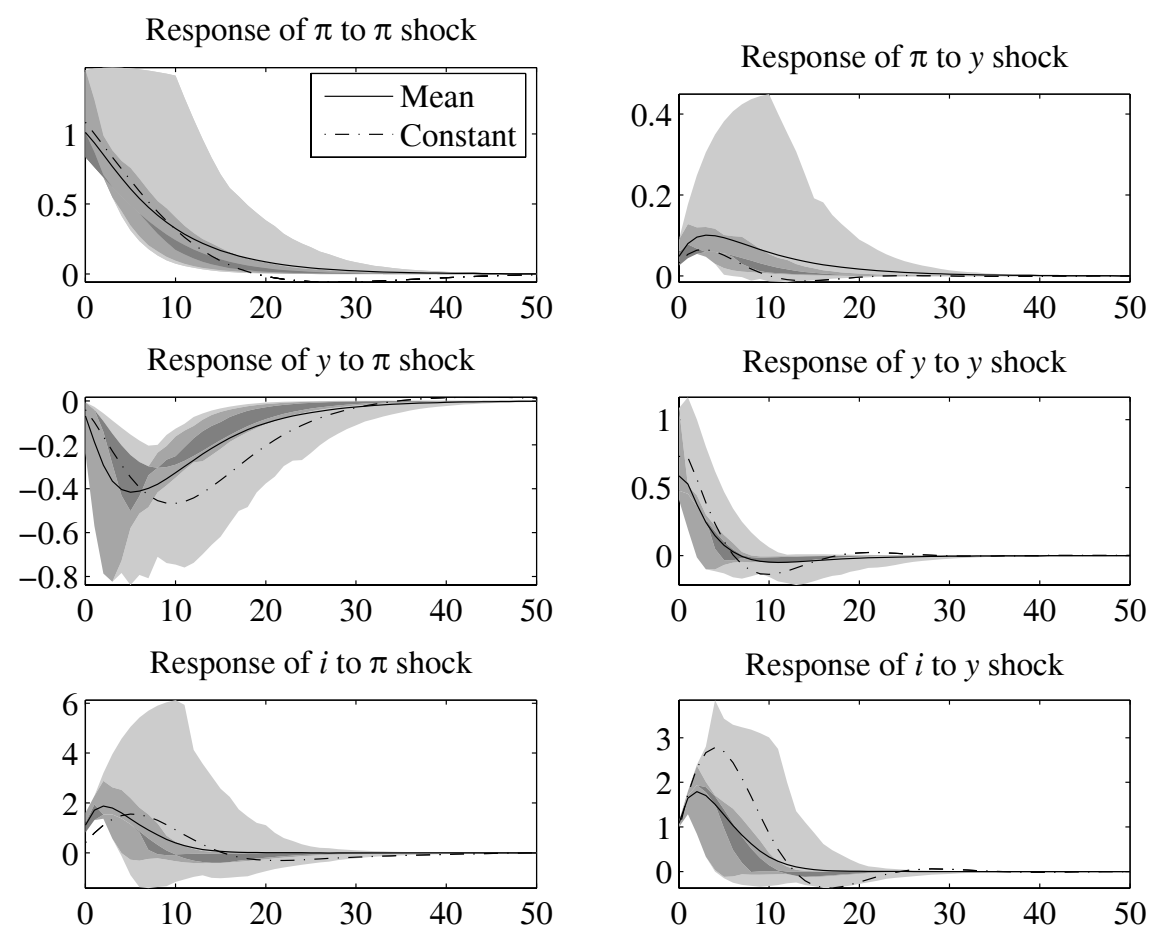

Figure 4.4: Unconditional impulse responses to shocks under the optimal policy for the modedependent Lindé model. Solid lines: Mean responses. Dark/medium/light grey bands: 30/60/90\% probability bands. Dashed lines: Optimal responses for the constant-coefficient model.

with earlier peak effects of the shocks which more rapidly return to zero. This is particularly the case for the instrument-rate responses, although the relative magnitudes differ somewhat with the type of the shock. For shocks to the output gap, most of the mass of the instrument-rate response distribution under model uncertainty lies below the response for the constant-coefficient model. For shocks to inflation, most of the distribution is consistent with larger and more prompt instrumentrate responses than for the constant-coefficient model. Once again, the distribution of the impulse responses is asymmetric, with the mean responses different from the median responses (the latter lie inside the dark gray bands), and again the tails of the distributions appear relatively wide. As in the RS model above, this is perhaps most noticeable for the inflation responses, where the center of the distribution lies below the constant-coefficient case but there is a relatively large right tail showing more significant and persistent responses. However in the Lindé model, the long-run behavior is better anchored as the distributions of responses in all cases collapse tightly around zero after roughly thirty quarters. 


\section{Arbitrary time-varying instrument rules and instrument paths}

In this section we derive the dynamics of the system, including the distribution of forecasts of relevant future variables, for arbitrary time-varying instrument rules. This includes time-varying instrument paths such as a constant instrument rate for arbitrary (but finitely many) periods, analogous to the constant-rate forecasts of the some central banks. We also specify the optimization problem for instrument rules in a given class. Furthermore, as we shall note, although we explicitly only deal with instrument rules, our method generalizes to arbitrary policy rules, including targeting rules (Svensson and Woodford [39]).

\subsection{Setup}

Consider implementing an arbitrary time-varying instrument rule during period $t=0,1, \ldots, T-1$ and implementing the optimal policy function from period $T$ on. Let the arbitrary instrument rule be conditionally linear but otherwise of the rather general form

$$
i_{t}=F_{\tilde{X} t j_{t}} \tilde{X}_{t}+F_{x t j_{t}} x_{t} \quad(0 \leq t \leq T-1)
$$

where $\tilde{X}_{t}$ denotes the $n_{\tilde{X}} \times 1$ vector $\left(X_{t}^{\prime}, \Xi_{t-1}^{\prime}\right)^{\prime}, F_{\tilde{X} t j_{t}}$ and $F_{x t j_{t}}$ are $\left(n_{i} \times n_{\tilde{X}}\right)$ and $\left(n_{i} \times n_{x}\right)$ matrices, respectively, which depend on both the period $t$ and the mode $j_{t}$. For added generality, we also allow a possible response to the forward-looking variables, $x_{t}$. Indeed, we could consider any arbitrary policy rule, including targeting rules, of the form

$$
\mathrm{E}_{t} H_{3, t+1, j_{t+1}} x_{t+1}=A_{31 t j_{t}} \tilde{X}_{t}+A_{32 t j_{t}} x_{t}+B_{3 t j_{t}} i_{t} \quad(0 \leq t \leq T-1)
$$

where $H_{3 t j}, A_{31 t j}, A_{32 t j}$, and $B_{3 t j}$ are potentially time-varying and mode-dependent matrices of the appropriate dimension (in particular, having $n_{i}$ rows and giving rise to $n_{i}$ independent equations, which is required to determine the instruments in each period).

If $F_{x t j_{t}} \equiv 0,(5.1)$ is an explicit instrument rule; that is, the instrument responds to predetermined variables only. ${ }^{13}$ If $F_{x t j_{t}} \not \equiv 0\left(F_{x t j_{t}} \neq 0\right.$ for some mode $j_{t}$ with positive probability), it is an implicit instrument rule; that is, the instrument depends also on forward-looking variables. In the latter case, there is a simultaneity problem, in that the instrument and the forward-looking variables are simultaneously determined. Thus, an implicit instrument rule can be interpreted as an equilibrium condition. As discussed in Svensson [36] and Svensson and Woodford [39], the implementation of an implicit instrument rule is problematic, since in practice a central bank can

\footnotetext{
${ }^{13}$ Note that policy functions and explicit instrument rules are the same.
} 
literally only respond to predetermined variables. ${ }^{14}$ We disregard these problems here, and consider (5.1) as just another equilibrium condition added to equations (2.1) and (2.2).

We can write (5.1) in the more general form

$$
0=F_{\tilde{X} t j_{t}} \tilde{X}_{t}+F_{\tilde{\imath} t j_{t}} \tilde{\imath}_{t} \quad(0 \leq t \leq T-1)
$$

where

$$
F_{\tilde{\imath} t j_{t}} \equiv\left[\begin{array}{lll}
F_{x t j_{t}}-I_{n_{i}} & 0_{n_{i} \times n_{x}}
\end{array}\right]
$$

where $\tilde{\imath}_{t} \equiv\left(x_{t}^{\prime}, i_{t}^{\prime}, \gamma_{t}^{\prime}\right)^{\prime}$ as in (2.9). Assume that the policy function shifts permanently to the optimal policy function (2.13) in period $T .{ }^{15}$ This is a reasonably general formulation. Since one of the elements of $X_{t}$ may be unity, (5.3) includes the case of an exogenous time-varying and modedependent instrument level for the first $T$ periods, including the case of a constant instrument level.

It follows from section 2 that there exists $\tilde{V}_{j}$ and $\tilde{w}_{j}(j=1, \ldots, n)$ such that, for $t \geq T$, the intertemporal loss for the dual saddlepoint problem satisfies

$$
\tilde{X}_{t}^{\prime} \tilde{V}_{j_{t}} \tilde{X}_{t}+\tilde{w}_{j_{t}} \equiv \max _{\gamma_{t}} \min _{\left(x_{t}, i_{t}\right)}\left\{\tilde{L}_{t}+\delta \mathrm{E}_{t}\left(\tilde{X}_{t+1}^{\prime} \tilde{V}_{j_{t+1}} \tilde{X}_{t+1}+\tilde{w}_{j_{t+1}}\right)\right\} \quad(t \geq T)
$$

subject to

$$
\tilde{X}_{t+1}=\tilde{A}_{j_{t+1}} \tilde{X}_{t}+\tilde{B}_{j_{t+1}} \tilde{\imath}_{t}+\tilde{C}_{j_{t+1}} \varepsilon_{t+1}
$$

and $\tilde{X}_{t}$ given $\left(\tilde{X}_{t}, \tilde{L}_{t}, \tilde{\imath}_{t}, \tilde{A}_{j_{t+1}}, \tilde{B}_{j_{t+1}}\right.$, and $\tilde{C}_{j_{t+1}}$ are defined as in (2.11) and (2.12)). Recall that this dual intertemporal loss is associated with the dual loss function, not the original loss function.

The recursive saddlepoint method of Marcet and Marimon [26] provides a simple and compact way to incorporate the fact that the equilibrium forward-looking variables $x_{t}$ and the Lagrange multiplier $\Xi_{t-1}$ will be affected by the constraint (5.1). Working backward, for $t=T-1, T-2, \ldots, 0$, we define $\tilde{V}_{t j_{t}}$ and $\tilde{w}_{t j_{t}}$ recursively from the saddlepoint problems:

$$
\tilde{X}_{t}^{\prime} \tilde{V}_{t j_{t}} \tilde{X}_{t}+\tilde{w}_{t j_{t}} \equiv \max _{\left(\gamma_{t}, \varphi_{t}\right)} \min _{\left(x_{t}, i_{t}\right)}\left\{\begin{array}{l}
\tilde{L}_{t}+\varphi_{t}^{\prime}\left(-F_{\tilde{X} t j_{t}} \tilde{X}_{t}-F_{\tilde{\imath} t j_{t}} \tilde{\imath}_{t}\right) \\
+\delta \mathrm{E}_{t}\left(\tilde{X}_{t+1}^{\prime} \tilde{V}_{t+1, j_{t+1}} \tilde{X}_{t+1}+\tilde{w}_{t+1, j_{t+1}}\right)
\end{array}\right\} \quad(0 \leq t \leq T-1),
$$

subject to (5.3) and (5.5), where $\tilde{V}_{T j_{t}} \equiv \tilde{V}_{j_{t}}$ and $\tilde{w}_{T j_{t}} \equiv \tilde{w}_{j_{t}}$. Here, $\varphi_{t}$ can be interpreted as an $n_{i} \times 1$ vector of Lagrange multipliers for the $n_{i}$ equations (5.3). Formally, (5.3) is added to the

\footnotetext{
${ }^{14}$ In practice, because of a complex and systematic decision process (Brash [7], Sims [31], Svensson [34]), the information modern central banks respond to is at least a few days old, and most of the information is one or several months old.

${ }_{15}$ Alternatively, the policy rule could shift to an arbitrary time-invariant policy rule for which a unique solution exists.
} 
equations (2.2) and the Lagrange multiplier $\gamma_{t}$ is augmented to $\left(\gamma_{t}^{\prime}, \varphi_{t}^{\prime}\right)^{\prime}$. Normally, the recursive saddlepoint method would then involve augmenting the Lagrange multiplier $\Xi_{t-1}$ to $\left(\Xi_{t-1}^{\prime}, \Phi_{t-1}^{\prime}\right)^{\prime}$, with the added dynamic equation

$$
\Phi_{t}=\varphi_{t}
$$

However, the augmented period loss is here

$$
\hat{L}_{t} \equiv \tilde{L}_{t}+\varphi_{t}^{\prime}\left(i_{t}-F_{\tilde{X} t j_{t}} \tilde{X}_{t}-F_{x t j_{t}} x_{t}\right)
$$

Since the analogue of $\mathrm{E}_{t} H_{t+1} x_{t+1}$, the left side of (5.3), is zero, there is no term including $\Phi_{t-1}^{\prime}$ augmented to the period loss. Hence, we do not need to consider $\Phi_{t-1}$ as an additional predetermined variable here. ${ }^{16}$

The solution determines the time- and mode-dependent optimal policy function $\tilde{F}_{t j_{t}}$,

$$
\tilde{\imath}_{t} \equiv\left[\begin{array}{c}
x_{t} \\
i_{t} \\
\gamma_{t}
\end{array}\right]=\tilde{F}_{t j_{t}} \tilde{X}_{t} \equiv\left[\begin{array}{c}
\tilde{F}_{x t j_{t}} \\
\tilde{F}_{i t j_{t}} \\
\tilde{F}_{\gamma t j_{t}}
\end{array}\right] \tilde{X}_{t} \quad(0 \leq t \leq T-1),
$$

where of course $i_{t}$ in $\tilde{\imath}_{t}$ satisfies (5.1). The interesting part of the solution is

$$
x_{t}=\tilde{F}_{x t j_{t}} \tilde{X}_{t},
$$

and $\tilde{F}_{x t j_{t}}$ and $\tilde{F}_{i t j_{t}}$ will satisfy

$$
\tilde{F}_{i t j_{t}} \equiv F_{\tilde{X} t j_{t}}+F_{x t j_{t}} \tilde{F}_{x t j_{t}}
$$

There is also a solution for $\varphi_{t}, \varphi_{t}=\tilde{F}_{\varphi t j_{t}} \tilde{X}_{t}$, but that solution is not needed for the intertemporal loss and the dynamics. It follows that the dynamics of $\tilde{X}_{t}$ satisfies

$$
\begin{aligned}
& \tilde{X}_{t+1}=M_{t j_{t} j_{t+1}} \tilde{X}_{t}+\tilde{C}_{j_{t+1}} \varepsilon_{t+1} \quad(0 \leq t \leq T-1), \\
& \tilde{X}_{t+1}=M_{j_{t} j_{t+1}} \tilde{X}_{t}+\tilde{C}_{j_{t+1}} \varepsilon_{t+1} \quad(t \geq T)
\end{aligned}
$$

where

$$
\begin{aligned}
M_{t j_{t} j_{t+1}} & \equiv \tilde{A}_{j_{t+1}}+\tilde{B}_{j_{t+1}} \tilde{F}_{t j_{t}} \quad(0 \leq t \leq T-1), \\
M_{j_{t} j_{t+1}} & \equiv \tilde{A}_{j_{t+1}}+\tilde{B}_{j_{t+1}} \tilde{F}_{j_{t}} \quad(t \geq T) .
\end{aligned}
$$

The intertemporal loss in period 0 for the dual period loss function (5.7) will be given by

$$
\tilde{X}_{0}^{\prime} \tilde{V}_{0 j_{0}} \tilde{X}_{0}+\tilde{w}_{0 j_{0}}
$$

\footnotetext{
${ }^{16}$ If we were considering the more general policy rule (5.2), the term $\mathrm{E}_{t} H_{3, t+1, j_{t+1}} x_{t}$ would require us to also consider $\Phi_{t-1}$ as an additional predetermined variable.
} 
However, this is not the intertemporal loss in period 0 for the original period loss function, (2.4). In order to find that, note that the intertemporal loss for the optimal policy for $t \geq T$ will be given by

$$
\tilde{X}_{t}^{\prime} V_{j_{t}} \tilde{X}_{t}+w_{j_{t}}
$$

where the matrix $V_{j}$ will satisfy the Lyapunov function (2.17) and the scalar $w_{j}$ will satisfy (2.18).

For $t=T-1, T-2, \ldots, 0$, we can define $V_{t j}$ and $w_{t j}$ recursively from the equations

$$
\begin{gathered}
\bar{W}_{t j} \equiv\left[\begin{array}{cc}
I & 0 \\
\tilde{F}_{x t j} \\
\tilde{F}_{i t j}
\end{array}\right]^{\prime} W_{j}\left[\begin{array}{cc}
I & 0 \\
\tilde{F}_{x t j} \\
\tilde{F}_{i t j}
\end{array}\right] . \\
V_{t j} \equiv \bar{W}_{t j}+\delta \sum_{k} P_{j k} M_{t j k}^{\prime} V_{t+1, k} M_{t j k}, \\
w_{t j t} \equiv \delta \sum_{k} P_{j k}\left[\operatorname{tr}\left(V_{t+1, k} \tilde{C}_{k} \tilde{C}_{k}^{\prime}\right)+w_{t+1, k}\right],
\end{gathered}
$$

where $V_{T j} \equiv V_{j}$ and $w_{T j} \equiv w_{j}{ }^{17}$

Then, the intertemporal loss in period 0 for the original period loss function (5.7) is

$$
\tilde{X}_{0}^{\prime} V_{0 j_{0}} \tilde{X}_{0}+w_{0 j_{0}}
$$

This corresponds to the loss under commitment in a timeless perspective when the instrument is restricted to fulfill (5.1) and shifts to the optimal policy in period $T$. That is, when the restriction (5.1) is removed in period $T$ and optimal policy is feasible, the commitment is not from scratch in period $T$ (in which case $\Xi_{T-1}$ would equal zero) but takes into account the previous Lagrange multiplier $\Xi_{T-1}$. In principle, this formulation also allows us to consider nonzero $\Xi_{-1}$ in period 0 .

The method described above also works for the backward-looking case, in which case

$$
\tilde{L}_{t} \equiv L_{t}
$$

and there are no variables $\gamma_{t}, x_{t}$, and $\Xi_{t-1}$ (equivalently, they are identically equal to zero). Then the intertemporal loss for the saddlepoint problem is equal to the intertemporal loss for the original problem.

Details about the computation of $\tilde{F}_{t j_{t}}$ and $\tilde{V}_{t j_{t}}$ are provided in appendix G.

\footnotetext{
${ }^{17}$ Note that we could also determine $V_{t j_{t}}$ and $w_{t j_{t}}$ relying on the analogue of the identity $(2.19)$ for this case.
} 


\subsection{Optimization}

Let $F_{t} \equiv\left\{F_{\tilde{X} t j_{t}}, F_{x t j_{t}}\right\}_{j_{t}=1}^{n}$ for $0 \leq t \leq T-1$, and let $F \equiv\left\{F_{t}\right\}_{t=0}^{T-1}$ denote the time- and modedependent policy functions for $0 \leq t \leq T-1$. We may assume that there is a feasible set $\mathcal{F}$ of such policy functions, so $F \in \mathcal{F}$. Then we can, in principle, consider choosing the policy functions optimally according to

$$
\min _{F \in \mathcal{F}}\left\{\tilde{X}_{0}^{\prime} V_{0 j_{0}}(F) \tilde{X}_{0}+w_{0 j_{0}}(F)\right\}
$$

where the notation emphasizes that $V_{0 j_{t}}$ and $w_{0 j_{t}}$ will depend on $F$. With the policy problem formulated this way, the optimal $F$ would depend on $\tilde{X}_{0}$ (including $\Xi_{-1}$ ) and $j_{0}$ as well as the covariance matrix $\tilde{C}_{k} \tilde{C}_{k}^{\prime}$ of the shocks $\tilde{C}_{k} \varepsilon_{t+1}$ to $\tilde{X}_{t+1}$ in mode $j_{t+1}=k(k=1, \ldots, n)$. That is, certainty equivalence does not necessarily hold for restricted classes of policy functions. If the class of time- and mode-dependent policy functions is sufficiently big, it would include the optimal policy function (2.13). If we were to add $\frac{1}{\delta} \Xi_{-1} H_{j_{0}} x_{0}$ to the period loss function in period 0 , the optimal policy function would then be a solution to (5.9).

Note that, if $\mathcal{F}$ is such that $F_{x t j_{t}} \neq 0$, the optimal $F$ is generally not unique. The reason is that for (5.8), if

$$
i_{t}=F_{\tilde{X} t j_{t}} \tilde{X}_{t}+F_{x t j_{t}} x_{t}
$$

is a solution, so is

$$
i_{t}=F_{\tilde{X} t j_{t}} \tilde{X}_{t}+F_{x t j_{t}} x_{t}+\zeta^{\prime}\left(x_{t}-\tilde{F}_{x j_{t}} \tilde{X}_{t}\right)=\left(F_{\tilde{X} t j_{t}}-\zeta^{\prime} \tilde{F}_{x j_{t}}\right) \tilde{X}_{t}+\left(F_{x t j_{t}}+\zeta^{\prime}\right) x_{t}
$$

for any $n_{x} \times 1$ vector $\zeta$.

\section{Arbitrary time-invariant instrument rules and optimal restricted instrument rules}

In this section we derive the dynamics of the system, including the distribution of forecasts of relevant future variables, for arbitrary time-invariant instrument rules. We also specify the optimization problem for time-invariant instrument rules in a given class. While this is a special case of the previous section, it is important in its own right and, in particular, allows a simpler algorithm. 


\subsection{Setup}

Consider an arbitrary time-invariant instrument rule,

$$
i_{t}=F_{\tilde{X} j_{t}} \tilde{X}_{t}+F_{x j_{t}} x_{t} \quad\left(j_{t}=1, \ldots, n\right),
$$

combined with (2.1) and (2.2). We can consider this as a special case of the time-varying instrument rules in section 5 , if we let $F_{\tilde{X} t j_{t}}=F_{\tilde{X} j_{t}}$ and $F_{x t j_{t}}=F_{x j_{t}}$ and apply the algorithm of that section by iterating from $t=T>t_{0}$ to $t=t_{0}$ but instead of stopping at $t_{0}=0$ letting $t_{0} \rightarrow-\infty$. In

practice, the iteration would stop when $\tilde{F}_{t j_{t}}$ and $\tilde{V}_{t j_{t}}$ have converged to $\tilde{F}_{j_{t}}$ and $\tilde{V}_{j_{t}}$. Partitioning $\tilde{F}_{j_{t}}$ conformably with $x_{t}, i_{t}$, and $\gamma_{t}$, we have

$$
\begin{aligned}
x_{t} & =\tilde{F}_{x j_{t}} \tilde{X}_{t}, \\
i_{t} & =F_{\tilde{X}_{t}} \tilde{X}_{t}+F_{x j_{t}} \tilde{F}_{x j_{t}} \tilde{X}_{t} \equiv \tilde{F}_{i j_{t}} \tilde{X}_{t}, \\
\tilde{X}_{t+1} & =M_{j_{t} j_{t+1}} \tilde{X}_{t}+\tilde{C}_{j_{t+1}} \varepsilon_{t+1} \quad\left(j_{t}=1, \ldots, n\right) .
\end{aligned}
$$

This gives rise to a probability distribution of $\tilde{X}_{t+\tau}, x_{t+\tau}$, and $i_{t+\tau}(\tau \geq 0)$ conditional on $\tilde{X}_{t}$ and $j_{t}$. This solution will be associated with a value function for the original period loss function,

$$
\tilde{X}_{t}^{\prime} V_{j_{t}} \tilde{X}_{t}+w_{j_{t}}
$$

\subsection{Optimization}

For a given restricted class $\mathcal{F}$ of instrument rules, we can consider the optimal restricted (timeinvariant) instrument rule $\hat{F}$, which minimizes an intertemporal loss function. This intertemporal loss function could be the conditional loss in a given period, say period 0 ,

$$
\hat{F} \equiv \arg \min _{F \in \mathcal{F}}\left\{\tilde{X}_{0}^{\prime} V_{j_{0}}(F) \tilde{X}_{0}+w_{j_{0}}(F)\right\}
$$

where the notation takes into account that $V_{j_{0}}(F)$ and $w_{j_{0}}(F)$ depend on $F \in \mathcal{F}$. This would make the optimal restricted time-invariant instrument rule depend on $\tilde{X}_{0}, j_{0}$, and the covariance matrices $\tilde{C}_{j} \tilde{C}_{j}^{\prime}$ of the shocks $\tilde{C}_{j} \varepsilon_{t+1}$ to $\tilde{X}_{t+1}$ in mode $j=1, \ldots, n$. Alternatively, the intertemporal loss function could be the unconditional mean of the period loss function:

$$
\hat{F}=\arg \min _{F \in \mathcal{F}} \mathrm{E}\left[L_{t}\right] .
$$

Note that

$$
\mathrm{E}\left[L_{t}\right]=(1-\delta) \mathrm{E}\left[\tilde{X}_{t}^{\prime} V_{j_{t}}(F) \tilde{X}_{t}+w_{j_{t}}(F)\right]
$$


Furthermore, the unconditional and conditional intertemporal loss are approximately the same when the intertemporal loss is scaled by $1-\delta$ and $\delta$ is close to one,

$$
\lim _{\delta \rightarrow 1-} \mathrm{E}_{t} \sum_{\tau=0}^{\infty}(1-\delta) \delta^{\tau} L_{t+\tau}=\mathrm{E}\left[L_{t}\right]=\lim _{\delta \rightarrow 1-}(1-\delta) \mathrm{E}\left[w_{j_{t}}\right]=\mathrm{E}\left[\operatorname{tr}\left(V_{j_{t}} \tilde{C}_{j_{t}} \tilde{C}_{j_{t}}^{\prime}\right)\right]=\sum_{j} \bar{p}_{j} \operatorname{tr}\left(V_{j} \tilde{C}_{j} \tilde{C}_{j}^{\prime}\right)
$$

where we recall that $\bar{p}=\left(\bar{p}_{1}, \ldots, \bar{p}_{n}\right)$ is the stationary distribution of modes.

\subsection{Optimal Taylor-type instrument rules in a forward-looking model}

We now apply the methods outlined above to derive optimal Taylor-type instrument rules in the estimated forward-looking model from section 4.2 above. In particular, we consider simple implicit instrument rules of the general form (disregarding the implementation problems mentioned above):

$$
i_{t}=f_{i j_{t}} i_{t-1}+f_{\pi j_{t}} \pi_{t}+f_{y j_{t}} y_{t} .
$$

This is a Taylor rule with interest-rate smoothing, whose coefficients may depend on the mode $j_{t}$ in period $t$. As special cases, we consider mode-independent Taylor rules, where the coefficients are constrained to be the same in all modes, and original Taylor rules without the smoothing coefficient $f_{i}$. We use the unconditional mean of the period loss, $\mathrm{E}\left[L_{t}\right]$, as the intertemporal loss function.

Note that, compared to (6.1), (6.2) implies a response to the predetermined variables $X_{t}$ rather

than to $\tilde{X}_{t} \equiv\left(X_{t}^{\prime}, \Xi_{t-1}^{\prime}\right)^{\prime}$. That is, we need not consider the Lagrange multiplier $\Xi_{t-1}$. Then the equilibrium solution will be of the simpler form

$$
\begin{aligned}
x_{t} & =G_{j_{t}} X_{t} \\
i_{t} & =\left(F_{X j_{t}}+F_{x j_{t}} G_{j_{t}}\right) X_{t} \equiv F_{j_{t}} X_{t} . \\
X_{t+1} & =\left(A_{11 j_{t+1}}+A_{12 j_{t+1}} G_{j_{t}}+B_{1} F_{j_{t}}\right) X_{t}+C_{j_{t+1}} \varepsilon_{t+1} \equiv M_{j_{t} j_{t+1}} X_{t}+C_{j_{t+1}} \varepsilon_{t+1},
\end{aligned}
$$

and not involve $\Xi_{t-1}$. This allows us to use a somewhat simpler algorithm than that discussed above. In appendix $\mathrm{H}$, we discuss in more detail this simpler algorithm for the calculations of the optimal time-invariant instrument rules and the associated losses.

The results are summarized in table 6.1, where we report the optimal response coefficients of the different forms of the instrument rules for the constant-coefficient and MJLQ versions of the model. Interestingly, we find that the optimal Taylor-type rules that are constrained to have the same responses in all modes are more aggressive in the MJLQ model than in the constantcoefficient model. This contrasts with the impulse responses for the optimal policy shown in table 4.4 above, where we found that the optimal policy in the MJLQ model had on average a slightly 


\begin{tabular}{|c|c|c|c|c|}
\hline Mode & $i_{t-1}$ & $\pi_{t}$ & $y_{t}$ & Loss \\
\hline \multicolumn{5}{|c|}{ Constant-coefficient model } \\
\hline \multicolumn{4}{|c|}{ Optimal policy function } & 11.10 \\
\hline- & - & 2.93 & 1.69 & 15.13 \\
\hline- & 0.89 & 0.80 & 0.83 & 11.67 \\
\hline \multicolumn{5}{|c|}{ MJLQ model } \\
\hline \multicolumn{4}{|c|}{ Optimal policy function } & 14.62 \\
\hline All modes & - & 3.97 & 2.07 & 20.96 \\
\hline Mode 1 & - & 3.01 & 3.10 & \\
\hline Mode 2 & - & 6.39 & 2.85 & 18.09 \\
\hline Mode 3 & - & 1.94 & 0.86 & \\
\hline All modes & 0.73 & 1.60 & 1.49 & 16.18 \\
\hline Mode 1 & 0.69 & 1.27 & 1.78 & \\
\hline Mode 2 & 0.87 & 3.06 & 2.40 & 15.32 \\
\hline Mode 3 & 0.81 & 1.16 & 0.83 & \\
\hline
\end{tabular}

Table 6.1: Optimal Taylor-type instrument rules for the estimated three-mode Lindé model.

more aggressive inflation response but a more attenuated output-gap response than in the constantcoefficient model. Similar conclusions apply for both the original and smoothed Taylor rules. This increased aggressiveness is further illustrated in figure 6.1. The figure shows the loss in the constantcoefficient and MJLQ models for mode-independent original and smoothed Taylor rules. For both smoothed and original Taylor rules, the loss function is more sensitive to variations in the inflation response coefficient of the policy rule. For both kinds of rules, performance in the MJLQ model is enhanced by more aggressive responses.

These results suggest that constraining the rules to react in the same way in all modes may push the optimal simple rules towards more aggressive responses. Moreover, as table 6.1 shows, the modeindependent original Taylor rules are suboptimal by a fairly sizeable margin. This stands in contrast to the constant-coefficient model, where the smoothed Taylor rule has a loss only slightly higher loss than the fully optimal policy. Thus we also consider mode-dependent original and smoothed Taylor rules, which are reported in the table. There we see that there is significant variation in the responses across modes, with mode 3 having the weakest responses (particularly for the output gap), while mode 2 has the strongest (particularly for the smoothed Taylor rules). In at least two of the three modes, the rules are again more aggressive than in the constant-coefficient model. Above we saw that the effects of uncertainty on policy, captured by comparison of the constant coefficient model to the MJLQ model, had ambiguous effects on optimal policy. In contrast, when the instrument rule is constrained to respond to fewer variables and not be history-dependent (that 

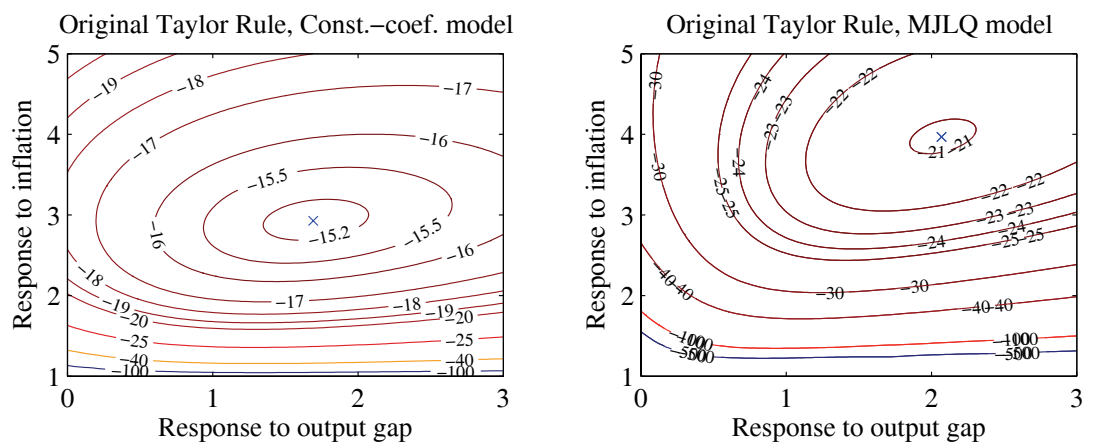

Smoothed Taylor Rule, $f_{i}=0.8$, Const. - coef. model
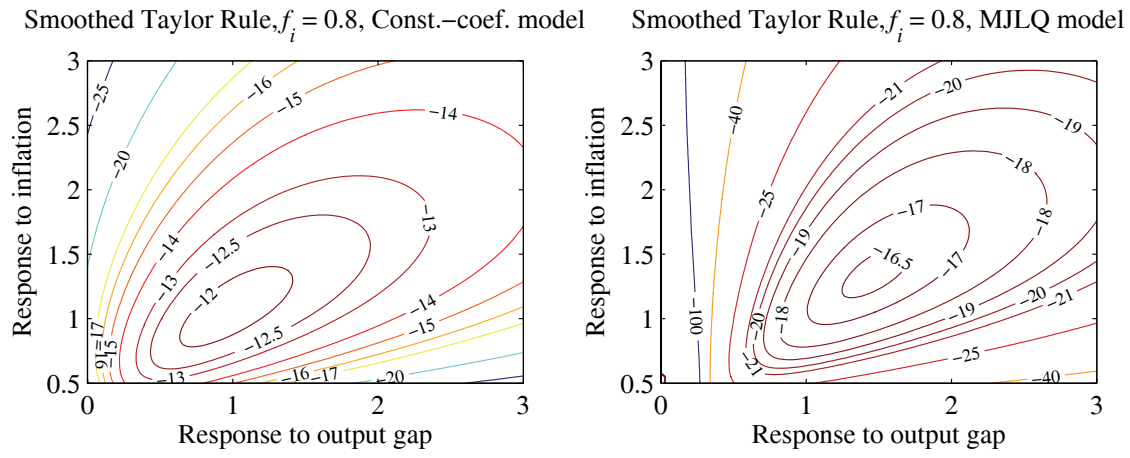

Figure 6.1: Contours of the loss function for the Lindé model under mode-independent Taylor-type instrument rules. Left column: Constant-coefficient model. Right column: MJLQ model. Top row: Original Taylor rules. Bottom row: Smoothed Taylor rules with $f_{i}=0.8$.

is, not respond to $\left.\Xi_{t-1}\right)$, uncertainty leads to more aggressive responses.

\section{Unobservable modes}

In this section we consider the case when the modes are not observable, showing how the optimal policy and value functions can be expressed as a function of the probability distribution of modes. Then we apply the results in our two estimated examples. As noted in the introduction, we do not consider the case where policymakers update their subjective distribution over modes based on observations. While this case is important, the learning which it implies introduces nonlinearities which destroy the tractability of the MJLQ framework. Instead, we assume here that the subjective distribution simply evolves according to the exogenous transition probabilities. 


\subsection{Optimal policy}

Assume that central bank cannot observe the actual mode in period $t$ and but believes that the distribution of modes in period $t$ is $p_{t} \equiv\left(p_{1 t}, \ldots, p_{n t}\right)$. Conditional on $p_{t}$ in period $t$, the distribution of the modes in period $t+\tau$ is given by

$$
p_{t+\tau}=p_{t} P^{\tau} \quad(\tau \geq 0) .
$$

With forward-looking variables, the dual model can be written

$$
\tilde{X}_{t+1}=\tilde{A}_{j_{t+1}} \tilde{X}_{t}+\tilde{B}_{j_{t+1}} \tilde{\imath}_{t j_{t}}+\tilde{C}_{j_{t+1}} \varepsilon_{t+1}
$$

where

$$
\tilde{\imath}_{t j_{t}} \equiv\left[\begin{array}{c}
x_{t j_{t}} \\
i_{t} \\
\gamma_{t j_{t}}
\end{array}\right]
$$

Note that $i_{t}$ will only depend on $p_{t}$ and be independent of $j_{t}$, since the instrument must reflect the central bank's information, whereas $x_{t}$ and $\gamma_{t}$ will depend on both $p_{t}$ and $j_{t}$. Appendix I shows that the optimal policy function can be written

$$
\tilde{\imath}_{t j_{t}} \equiv\left[\begin{array}{c}
x_{t j_{t}} \\
i_{t} \\
\gamma_{t j_{t}}
\end{array}\right]=\left[\begin{array}{c}
F_{x}\left(p_{t}\right)_{j_{t}} \\
F_{i}\left(p_{t}\right) \\
F_{\gamma}\left(p_{t}\right)_{j_{t}}
\end{array}\right] \tilde{X}_{t} \equiv F\left(p_{t}\right)_{j_{t}} \tilde{X}_{t} .
$$

The dynamics of the predetermined variables will follow

$$
\tilde{X}_{t+1}=M\left(p_{t}\right)_{j_{t} j_{t+1}} \tilde{X}_{t}+\tilde{C}_{j_{t+1}} \varepsilon_{t+1}
$$

where

$$
M\left(p_{t}\right)_{j k} \equiv \tilde{A}_{k}+\tilde{B}_{k} F\left(p_{t}\right)_{j}
$$

The value function for the original problem can be written

$$
\tilde{X}_{t}^{\prime} V\left(p_{t}\right) \tilde{X}_{t}+w\left(p_{t}\right)
$$

Appendix I shows how the functions $F\left(p_{t}\right)_{j}, V\left(p_{t}\right)$, and $w\left(p_{t}\right)$ can be computed by modifying the iterations specified in appendix B. Computing the functions $F\left(p_{t}\right)_{j}$ and $V\left(p_{t}\right)$ for all feasible values of $p_{t}$ requires standard function-approximation methods. However, as shown in appendix B, computing the functions for a particular value $p_{t}=\tilde{p}_{t}$ is straightforward. ${ }^{18}$

\footnotetext{
${ }^{18}$ Consider the degenerate distributions, $p_{t}=e_{j}$ where $e_{j}$ is the distribution where $p_{j}=1, p_{k}=0(k \neq j)$. That is, $p_{t}=e_{j}$ corresponds to the case when the mode $j$ is observed in period $t$. Note that $V\left(e_{j}\right) \neq V_{j}$ and $F\left(e_{j}\right)_{j} \neq$ $F_{j}$, where $V_{j}$ and $F_{j}(j=1, \ldots, n)$ denote the value function and optimal policy function matrices for the case when the modes are observed in each period. The reason is that even if $p_{t}=e_{j}$ and the mode is observed in this period, the distribution of the modes in the next period will be $p_{t+1}=e_{j} P=\left(P_{j 1}, P_{j 2}, \ldots, P_{j n}\right)$ and the modes will not be observed in the next period. In contrast, $V_{j}$ and $F_{j}$ are derived under the assumption that the modes are observed in this period as well as every future period.
} 
Consider now the optimal decision of a central bank in a given period $t$, with a given realization of the predetermined variables, $\tilde{X}_{t}$, and a given probability distribution of the modes, $p_{t}$. The probability distribution of the modes $\tau$ periods ahead is then given by (7.1). It follows that the optimal policy function for period $t+\tau(\tau \geq 0)$ is time-varying and can be written

$$
i_{t+\tau}=F_{i, t+\tau} \tilde{X}_{t+\tau} \quad(\tau \geq 0)
$$

where

$$
F_{i, t+\tau} \equiv F_{i}\left(p_{t} P^{\tau}\right)
$$

Hence, this situation is a special case of that discussed in section 5, where the policy function is timevarying but independent of the mode. That is, the instrument rule in (5.1) satisfies $F_{\tilde{X}, t+\tau, j_{t+\tau}} \equiv$ $F_{i, t+\tau}$ and $F_{x, t+\tau, j_{t+\tau}} \equiv 0$.

\subsection{Examples}

In this section we reconsider the two examples from section 4 above, now under the assumption that the modes are unobservable. We suppose that the central bank has an initial distribution over the modes which is equal to the stationary distribution $\bar{p}$. From equation (7.1), we see that the stationary distribution is also the central bank's distribution of the future modes. We then apply the algorithms described in appendix I to find the optimal policies. As in the observable-mode case, we represent the solutions via impulse responses from 10,000 simulations, drawing the initial mode from the stationary distribution and tracing out the distribution as the modes vary (now in an unobservable manner) over time.

\begin{tabular}{c|rrrrrrrrr}
\hline \hline Case & $\pi_{t}$ & $\pi_{t-1}$ & $\pi_{t-2}$ & $\pi_{t-3}$ & $y_{t}$ & $y_{t-1}$ & $i_{t-1}$ & $i_{t-2}$ & $i_{t-3}$ \\
\hline Constant & 0.9921 & 0.3465 & 0.4273 & 0.1381 & 1.7974 & -0.4639 & 0.3713 & -0.0899 & -0.0456 \\
\hline Unobserved & 1.1828 & 0.3610 & 0.7304 & 0.1861 & 1.9103 & -1.0563 & 0.1538 & -0.1573 & -0.0758 \\
\hline \hline
\end{tabular}

Table 7.1: Optimal policy functions for the constant-coefficient and unobserved-mode versions of the Rudebusch-Svensson model.

In table 7.1 we show the optimal policy functions for the constant-coefficient and unobservablemode versions of the RS model from section 4.1 above. In figure 7.1 we plot the impulse responses. The distributions of the impulse responses are again asymmetric, with the mean impulse responses different from the median ones. Compared to the observable-mode case in figure 4.2 above, we see that the mean policy responses are longer lasting, if not noticeably more aggressive at the start. However most of the center of the distribution is consistent with smaller responses, with the mean 

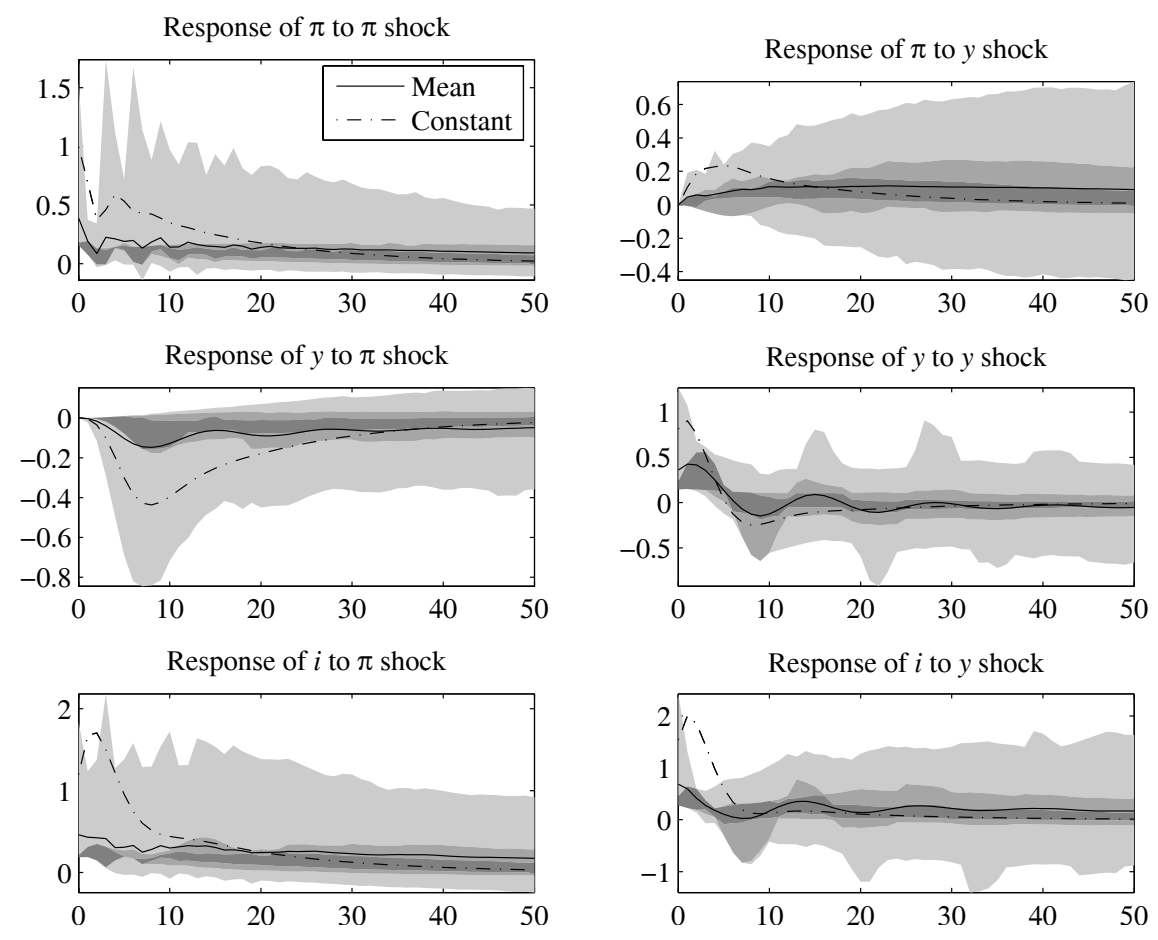

Figure 7.1: Unconditional impulse responses to shocks under the optimal policy for the unobservedmode version of the Rudebusch-Svensson model. Solid lines: Mean responses. Dark/medium/light grey bands: 30/60/90\% probability bands. Dashed lines: Optimal responses for constant coefficients.

reflecting the very wide tails. Further, although the impulse-response distributions become relatively concentrated around zero over time, the tails remain quite wide after the full 50 quarters shown. Since the coefficients of the mode-dependent optimal policy functions change dramatically across modes, being restricted to mode-independent (although distribution-dependent) policy functions limits the possibility to stabilize the economy and generates wider distributions.

\begin{tabular}{c|rrrrrrrr}
\hline \hline Case & $\pi_{t-1}$ & $y_{t-1}$ & $y_{t-2}$ & $i_{t-1}$ & $\varepsilon_{\pi t}$ & $\varepsilon_{y t}$ & $\Xi_{\pi, t-1}$ & $\Xi_{y, t-1}$ \\
\hline Constant & 0.3552 & 1.0714 & -0.2231 & 0.7853 & 0.6975 & 2.2437 & 0.0024 & 0.0182 \\
\hline Unobserved & 1.0987 & 1.7439 & -0.2497 & 0.4788 & 1.7987 & 2.1787 & 0.0059 & 0.0194 \\
\hline \hline
\end{tabular}

Table 7.2: Optimal policy functions of the constant-coefficient and unobserved-mode versions the Lindé model.

In table 7.2 we show the optimal policy functions for constant-coefficient and the unobservablemode versions of the Lindé model from section 4.2 above. In figure 7.2 we plot the impulse responses. 

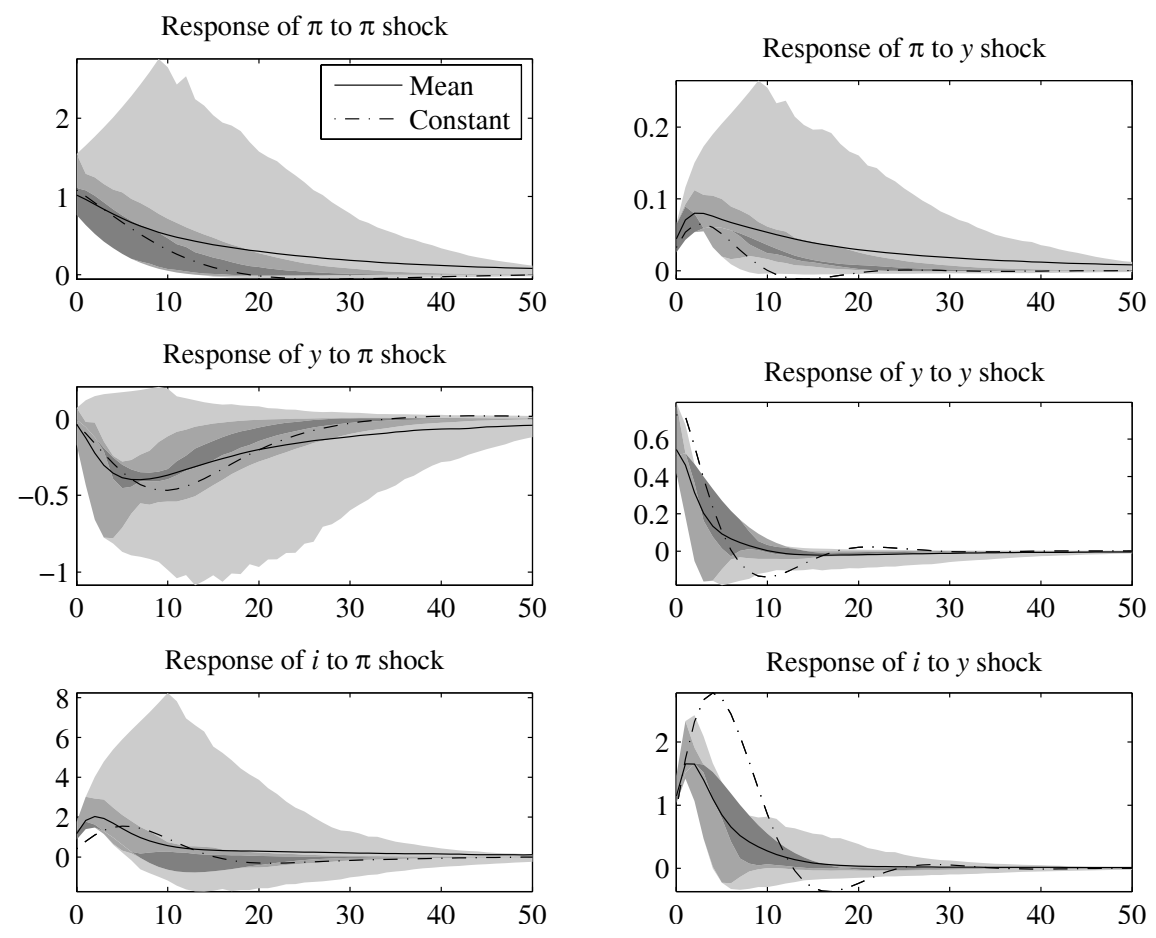

Figure 7.2: Unconditional impulse responses to shocks under the optimal policy for the unobservedmode version of the Lindé model. Solid lines: Mean responses. Dark/medium/light grey bands: 30/60/90\% probability bands. Dashed lines: Optimal responses for constant coefficients.

As for the backward-looking model above, unobservability of the modes has some effects on the distribution of impulse responses in this forward-looking model. Comparing to figure 4.4 above, we see that, although the mean and median policy responses are similar in the two cases, the tails are wider for responses to inflation shocks, whereas they are tighter for output-gap shocks. As we have seen above, the optimal policy in the observable-mode case reacts more strongly in some of the modes (particularly in mode 2) and hence the different distributions in the observable-mode case reflects the variation in the policy across modes. In the unobservable-mode case, the optimal policy averages across modes. This averaging leads to slower convergence of the distributions over time, although not to the same sustained dynamics as in the backward-looking model. Apart from the other differences across the forward and backward-looking models, it seems, also when the modes cannot be observed, that expectations play a key role in stabilizing the economy. 


\section{Conclusions}

This paper demonstrates that the Markov jump-linear-quadratic (MJLQ) framework is a very flexible and powerful tool for the analysis and determination of optimal policy under model uncertainty. It provides a very tractable way of handling the absence of certainty equivalence that is an important aspect of model uncertainty. Our approach builds on the control-theory literature, for instance, Costa, Fragoso, and Marques [11], which has explored many properties of the MJLQ framework. That literature uses recursive methods and does not consider forward-looking variables. However the forward-looking variables characteristic of rational expectations make the models nonrecursive. We show that the recursive saddlepoint method of Marcet and Marimon [26] can be applied to this problem which allows us to use recursive methods, and hence to solve relatively general models.

We show that our framework can incorporate a large variety of different configurations of uncertainty. We provide algorithms to derive the optimal policy and value functions. We apply the framework to two examples: regime-switching variants of two empirical models of the US economy, the backward-looking model of Rudebusch and Svensson [30] and the forward-looking New Keynesian model of Lindé [24]. We also show how the dynamics of the model can be specified for arbitrary time-varying or time-invariant policy functions, including exogenous instrument paths such as a constant instrument rate, and we discuss how to optimize over restricted instrument rules. Finally, we show how the framework an be adapted to a situation with unobservable modes, arguably the most realistic situation for policy. In the examples we study, we find some substantial deviations from certainty equivalence. In some cases, we find support for the common intuition that uncertainty should make policy more cautious, but this is not a general result. Overall, our results illustrate the importance of considering the entire distribution of future outcomes.

The MJLQ framework makes it possible to provide advice on optimal monetary policy for a large variety of different configurations of model uncertainty. The framework also makes it possible to incorporate different kinds of central-bank judgment - information, knowledge, and views outside the scope of a particular model - about the kind and degree of model uncertainty. Furthermore, the framework can incorporate the kind of central-bank judgment about additive future deviationsadd factors - that is discussed in Svensson [36] and Svensson and Tetlow [38].

While the particular examples we study in this paper are informative, they are only a small sample of the applications which can be analyzed with our approach. In addition to the further examples outlined above and sketched in the paper, some natural applications would embed the 
different specifications of fully specified dynamic stochastic general equilibrium models as modes in the MJLQ setting. We could thus incorporate uncertainty about the structure of the economy, such as different forms of price or wage setting (as discussed in Levin, Onatski, Williams, and Williams [23]). Alternative specifications could also capture uncertainty about the low-frequency behavior of the key driving processes, which could describe potential productivity slowdowns (as in, for example, Kahn and Rich [20]) or moderations in overall volatility (as in McConnell and Perez-Quiros [27] and Stock and Watson [33]). This would help address a drawback of our results so far, that the different modes are not readily interpretable in terms of fundamentals. Instead, by having the modes represent different structural models there will be natural restrictions on the parameters and how they co-move. This would allow us to study monetary policy with unknown and potentially time-varying structural models.

Overall, our results point to some important changes from approaches considering additive uncertainty. In the "mean forecast targeting" applications in Svensson [36] and Svensson and Tetlow [38], certainty equivalence is preserved, since the uncertainty is restricted to additive future stochastic deviations in the model's equations. With certainty equivalence, only the means of future variables matter for policy, and optimal policy can be derived as if there were no uncertainty about those means. Furthermore, the optimal mean projection of future target variables and the instrument can be calculated in one step, and those projections-including the optimal mean instrument path - are the natural objects for policy discussion. There is no need to use recursive methods, and there is no need to specify the optimal policy function for the policy makers (the explicit policy function is also a high-dimensional vector that is not easy to interpret). Instead, the policy discussion can be conducted with the help of computer-generated graphs of projections of the target variables and the instrument under alternative assumptions, weights in the monetary-policy loss function, and central-bank judgments.

In the absence of certainty equivalence, mean forecast targeting is in principle no longer sufficient. The whole distribution of future target variables matters for policy, and the optimal instrument decision should in principle take this into account. The optimal policy plan should be chosen such that the whole distribution, rather than the mean, of the future target variables "looks good." The central bank should engage in "distribution forecast targeting" rather than mean forecast targeting. The application of the MJLQ framework in this paper to model uncertainty and certainty non-equivalence indicates that recursive methods and the explicit policy function are relatively more useful for the derivation of the optimal policy than under certainty equivalence, perhaps 
even necessary. Still, the resulting distributions of future target variables and instruments under alternative assumptions can conveniently be illustrated and presented to policy makers in the form of graphs, although graphs of distributions rather than of means. 


\section{Appendix}

\section{A Incorporating central-bank judgment}

In order to incorporate (additive) central-bank judgment as in Svensson [36], consider the model

$$
\begin{aligned}
X_{t+1} & =A_{11, t+1} X_{t}+A_{12, t+1} x_{t}+B_{1, t+1} i_{t}+C_{t+1} z_{t+1}, \\
\mathrm{E}_{t} H_{t+1} x_{t+1} & =A_{21, t} X_{t}+A_{22, t} x_{t}+B_{2, t} i_{t}
\end{aligned}
$$

where $z_{t}$, the (additive) deviation, is a an exogenous $n_{z} \times 1$ vector stochastic process. Assume that $z_{t}$ satisfies

$$
z_{t+1}=\varepsilon_{t+1}+\sum_{j=1}^{T} \varepsilon_{t+1, t+1-j}
$$

for given $T \geq 0$, where $\left(\varepsilon_{t}^{\prime}, \varepsilon^{t \prime}\right)^{\prime} \equiv\left(\varepsilon_{t}^{\prime}, \varepsilon_{t+1, t}^{\prime}, \ldots, \varepsilon_{t+T, t}^{\prime}\right)^{\prime}$ is a zero-mean i.i.d. random $(T+1) n_{z} \times 1$ vector realized in the beginning of period $t$ and called the innovation in period $t$. For $T=0$, $z_{t+1}=\varepsilon_{t+1}$ is a simple i.i.d. disturbance. For $T>0$, the deviation is a version of a moving-average process.

The dynamics of the deviation can be written

$$
\left[\begin{array}{c}
z_{t+1} \\
z^{t+1}
\end{array}\right]=A_{z}\left[\begin{array}{c}
z_{t} \\
z^{t}
\end{array}\right]+\left[\begin{array}{c}
\varepsilon_{t+1} \\
\varepsilon^{t+1}
\end{array}\right]
$$

where $z^{t} \equiv\left(\mathrm{E}_{t} z_{t+1}^{\prime}, \mathrm{E}_{t} z_{t+2}^{\prime}, \ldots, \mathrm{E}_{t} z_{t+T}\right)^{\prime}$ can be interpreted as the central bank's (additive) judgment in period $t$ and the $(T+1) n_{z} \times(T+1) n_{z}$ matrix $A_{z}$ is defined as

$$
A_{z} \equiv\left[\begin{array}{ccc}
0_{n_{z} \times n_{z}} & I_{n_{z}} & 0_{n_{z} \times(T-1) n_{z}} \\
0_{(T-1) n_{z} \times n_{z}} & 0_{(T-1) n_{z} \times n_{z}} & I_{(T-1) n_{z}} \\
0_{n_{z} \times n_{z}} & 0_{n_{z} \times n_{z}} & 0_{n_{z} \times(T-1) n_{z}}
\end{array}\right] \equiv\left[\begin{array}{cc}
0 & A_{z 21} \\
0 & A_{z 22}
\end{array}\right] ;
$$

in the second identity $A_{z}$ is partitioned conformably with $z_{t}$ and $z^{t}$. Hence $z^{t}$ is the central bank's mean projection of future deviations, and $\varepsilon^{t}$ can be interpreted as the new information the central bank receives in period $t$ about those future deviations. ${ }^{19}$

It follows that the model can be written in the state-space form (2.1) and (2.2) as

$$
\begin{aligned}
{\left[\begin{array}{c}
X_{t+1} \\
z_{t+1} \\
z^{t+1}
\end{array}\right] } & =\hat{A}_{11, t+1}\left[\begin{array}{c}
X_{t} \\
z_{t} \\
z^{t}
\end{array}\right]+\hat{A}_{12, t+1} x_{t}+\hat{B}_{1, t+1} i_{t}+\hat{C}_{t+1}\left[\begin{array}{c}
\varepsilon_{t+1} \\
\varepsilon^{t+1}
\end{array}\right], \\
\mathrm{E}_{t} H_{t+1} x_{t+1} & =\hat{A}_{21, t}\left[\begin{array}{c}
X_{t} \\
z_{t} \\
z^{t}
\end{array}\right]+A_{22, t} x_{t}+B_{2, t} i_{t},
\end{aligned}
$$

\footnotetext{
${ }^{19}$ The graphs in Svensson [36] can be seen as impulse responses to $\varepsilon^{t}$.
} 
where

$$
\begin{gathered}
\hat{A}_{11, t+1} \equiv\left[\begin{array}{ccc}
A_{11, t+1} & 0 & C_{t+1} A_{z 21} \\
0 & 0 & A_{z 21} \\
0 & 0 & A_{z 22}
\end{array}\right], \quad \hat{A}_{21, t+1} \equiv\left[\begin{array}{c}
A_{21, t+1} \\
0 \\
0
\end{array}\right], \\
\hat{B}_{1, t+1} \equiv\left[\begin{array}{c}
B_{1, t+1} \\
0 \\
0
\end{array}\right], \quad \hat{C}_{t+1} \equiv\left[\begin{array}{cc}
0 & C_{t+1} \\
I_{n_{z}} & 0 \\
0 & I_{n_{z}}
\end{array}\right],
\end{gathered}
$$

and the new predetermined variables are $\left(X_{t}^{\prime}, z_{t}^{\prime}, z^{t \prime}\right)^{\prime}$.

\section{B An algorithm for the value function and optimal policy function}

Consider the dual saddlepoint problem of (2.6) in period $t$, subject to (2.7), (2.8), and $\tilde{X}_{t}$ given. Let us use the notation $Z_{t}=Z_{j_{t}}$ for any matrix $Z$ that is a function of the mode $j_{t}$, and let the matrix $\tilde{W}_{t}=\tilde{W}_{j_{t}}$ be partitioned conformably with $\tilde{X}_{t}$ and $\tilde{\imath}_{t}$ as

$$
\tilde{W}_{t} \equiv\left[\begin{array}{cc}
Q_{t} & N_{t} \\
N_{t}^{\prime} & R_{t}
\end{array}\right]
$$

We use that the value function for the dual problem will be quadratic and can be written

$$
\tilde{X}_{t}^{\prime} \tilde{V}_{t} \tilde{X}_{t}+\tilde{w}_{t}
$$

where $\tilde{V}_{t}$ is a matrix and $\tilde{w}_{t}$ a scalar. It will satisfy the Bellman equation

$$
\tilde{X}_{t}^{\prime} \tilde{V}_{t} \tilde{X}_{t}+\tilde{w}_{t}=\max _{\gamma_{t}} \min _{\left(x_{t}, i_{t}\right)}\left\{\tilde{X}_{t}^{\prime} Q_{t} \tilde{X}_{t}+2 \tilde{X}_{t}^{\prime} N_{t} \tilde{\tau}_{t}+\tilde{\imath}_{t}^{\prime} R_{t} \tilde{\tau}_{t}+\delta \mathrm{E}_{t}\left(\tilde{X}_{t+1}^{\prime} \tilde{V}_{t+1} \tilde{X}_{t+1}+\tilde{w}_{t+1}\right)\right\},
$$

where $\tilde{X}_{t+1}$ is given by $(2.8)$ and $\mathrm{E}_{t}$ refers to the expectations conditional on $\tilde{X}_{t}$ and $j_{t}$.

The first-order condition with respect to $\tilde{\imath}_{t}$ is

$$
\tilde{X}_{t}^{\prime} N_{t}+\tilde{\imath}_{t}^{\prime} R_{t}+\delta \tilde{X}_{t}^{\prime} \mathrm{E}_{t} \tilde{A}_{t+1}^{\prime} \tilde{V}_{t+1} \tilde{B}_{t+1}+\delta \tilde{\imath}_{t}^{\prime} \mathrm{E}_{t} \tilde{B}_{t+1}^{\prime} \tilde{V}_{t+1} \tilde{B}_{t+1}=0
$$

which can be written

$$
J_{t} \tilde{\imath}_{t}+K_{t} \tilde{X}_{t}=0
$$

where

$$
\begin{aligned}
J_{t} & \equiv R_{t}+\delta \mathrm{E}_{t} \tilde{B}_{t+1}^{\prime} \tilde{V}_{t+1} \tilde{B}_{t+1}, \\
K_{t} & \equiv N_{t}^{\prime}+\delta \mathrm{E}_{t} \tilde{B}_{t+1}^{\prime} \tilde{V}_{t+1} \tilde{A}_{t+1} .
\end{aligned}
$$

This leads to the optimal policy function

$$
\tilde{\imath}_{t}=F_{t} \tilde{X}_{t}
$$


where

$$
F_{t} \equiv-J_{t}^{-1} K_{t}
$$

Furthermore, the value function satisfies

$\tilde{X}_{t}^{\prime} \tilde{V}_{t} \tilde{X}_{t} \equiv \tilde{X}_{t}^{\prime} Q_{t} \tilde{X}_{t}+2 \tilde{X}_{t}^{\prime} N_{t} F_{t} \tilde{X}_{t}+\tilde{X}_{t}^{\prime} F_{t}^{\prime} R_{t} F_{t} \tilde{X}_{t}+\delta \tilde{X}_{t}^{\prime} \mathrm{E}_{t}\left[\left(\tilde{A}_{t+1}^{\prime}+F_{t}^{\prime} \tilde{B}_{t+1}^{\prime}\right) \tilde{V}_{t+1}\left(\tilde{A}_{t+1}+\tilde{B}_{t+1} F_{t}\right)\right] \tilde{X}_{t}$.

This implies

$$
\tilde{V}_{t}=Q_{t}+N_{t} F_{t}+F_{t}^{\prime} N_{t}^{\prime}+F_{t}^{\prime} R_{t} F_{t}+\delta \mathrm{E}_{t}\left[\left(\tilde{A}_{t+1}^{\prime}+F_{t}^{\prime} \tilde{B}_{t+1}^{\prime}\right) \tilde{V}_{t+1}\left(\tilde{A}_{t+1}+\tilde{B}_{t+1} F_{t}\right)\right]
$$

which can be simplified to the Riccati equation

$$
\tilde{V}_{t}=Q_{t}+\delta \mathrm{E}_{t} \tilde{A}_{t+1}^{\prime} \tilde{V}_{t+1} \tilde{A}_{t+1}-K_{t}^{\prime} J_{t}^{-1} K_{t}
$$

Equations (B.1), (B.2), and (B.5) show how $\tilde{V}_{t+1}=\tilde{V}_{j_{t+1}}$ for $j_{t+1}=1, \ldots, n$ is mapped into $\tilde{V}_{t}=\tilde{V}_{j_{t}}$ for $j_{t}=1, \ldots, n$.

Iteration backwards of (B.4) and (B.5) from any constant positive semidefinite matrix $\tilde{V}$ should converge to stationary matrices functions $F_{j}$ and $\tilde{V}_{j}(j=1, \ldots, n)$, where $\tilde{V}_{j}$ satisfies the Riccati equation (B.5) with (B.1) and (B.2).

Taking account of the finite number of modes, we have

$$
\begin{aligned}
F_{j} & \equiv-J_{j}^{-1} K_{j} \\
J_{j} & \equiv R_{j}+\delta \sum_{k=1}^{n} P_{j k} \tilde{B}_{k}^{\prime} \tilde{V}_{k} \tilde{B}_{k} \\
K_{j} & \equiv N_{j}^{\prime}+\delta \sum_{k=1}^{n} P_{j k} \tilde{B}_{k}^{\prime} \tilde{V}_{k} \tilde{A}_{k}, \\
\tilde{V}_{j} & =Q_{j}+\delta \sum_{k=1}^{n} P_{j k} \tilde{A}_{k}^{\prime} \tilde{V}_{k} \tilde{A}_{k}-K_{j}^{\prime} J_{j}^{-1} K_{j} \quad(j=1, \ldots, n),
\end{aligned}
$$

where $P_{j k}$ is the transition probability from $j_{t}=j$ to $j_{t+1}=k$.

The scalars $\tilde{w}_{j}$ solve the equations

$$
\tilde{w}_{j}=\delta \sum_{k} P_{j k}\left[\operatorname{tr}\left(\tilde{V}_{k} \tilde{C}_{k} \tilde{C}_{k}^{\prime}\right)+\tilde{w}_{k}\right]
$$

Thus determining the optimal policy function (B.3) reduces to solving a system of coupled algebraic Riccati equations (B.6). In order to solve this system numerically, we adapt the algorithm of do Val, Geromel, and Costa [14]. In a very similar problem, they show how the coupled Riccati equations can be uncoupled for numerical solution. ${ }^{20}$

\footnotetext{
${ }^{20}$ In their problem, the matrices $A$ and $B$ next period are known in the current period, so the averaging in the Riccati equation is only over the $V_{j}$ matrices.
} 
The algorithm consists of the following steps:

1. Define $\hat{A}_{j}=\sqrt{P_{j j}} \tilde{A}_{j}, \hat{B}_{j}=\sqrt{P_{j j}} \tilde{B}_{j}$ and initialize $\tilde{V}_{j}^{0}=0, j=1, \ldots, n$.

2. Then at each iteration $l=0,1, \ldots$, for each $j$ define:

$$
\begin{aligned}
& \hat{Q}_{j}=Q_{j}+\delta \sum_{k \neq j} P_{j k} \tilde{A}_{k}^{\prime} \tilde{V}_{k}^{l} \tilde{A}_{k} \\
& \hat{R}_{j}=R_{j}+\delta \sum_{k \neq j} P_{j k} \tilde{B}_{k}^{\prime} \tilde{V}_{k}^{l} \tilde{B}_{k} \\
& \hat{N}_{j}=N_{j}+\delta \sum_{k \neq j} P_{j k} \tilde{A}_{k}^{\prime} \tilde{V}_{k}^{l} \tilde{B}_{k} .
\end{aligned}
$$

Then for each $j$ solve the standard Riccati equation for the problem with matrices $\left(\hat{A}_{j}, \hat{B}_{j}, \hat{Q}_{j}\right.$, $\left.\hat{R}_{j}, \hat{N}_{j}\right)$. Note that these are uncoupled since $\tilde{V}_{k}^{l}$ is known. Call the solution $\tilde{V}_{j}^{l+1}$.

3. Check $\sum_{j=1}^{n}\left\|\tilde{V}_{j}^{l+1}-\tilde{V}_{j}^{l}\right\|$. If this is lower then a tolerance, stop. Otherwise, return to step 2.

do Val, Geromel, and Costa [14] show that the sequence of matrices $\tilde{V}_{j}^{l}$ converges to the solution of (B.6) as $l \rightarrow \infty$. In order to understand the algorithm, recall that, in the standard linearquadratic regulator (LQR) problem (Anderson, Hansen, McGrattan, and Sargent [1] and Ljungqvist and Sargent [25]), we have

$$
\begin{aligned}
F & \equiv-J^{-1} K \\
J & \equiv R+\delta B^{\prime} V B \\
K & \equiv N^{\prime}+\delta B^{\prime} V A, \\
V & =Q+\delta A^{\prime} V A-K^{\prime} J^{-1} K .
\end{aligned}
$$

If we can redefine the matrices so the equations conform to the standard case, we can use the standard algorithm for the LQR problem to find $F_{j}$ and $V_{j}$. The above definitions allow us to write

$$
\begin{aligned}
F_{j} & \equiv-J_{j}^{-1} K_{j}, \\
J_{j} & \equiv \hat{R}_{j}+\delta \hat{B}_{j}^{\prime} \tilde{V}_{j} \hat{B}_{j}, \\
K_{j} & \equiv \hat{N}_{j}^{\prime}+\delta \hat{B}_{j}^{\prime} \tilde{V}_{j} \hat{A}_{j}, \\
\tilde{V}_{j} & =\hat{Q}_{j}+\delta \hat{A}_{j}^{\prime} \tilde{V}_{j} \hat{A}_{j}-K_{j}^{\prime} J_{j}^{-1} K_{j} \quad(j=1, \ldots, n),
\end{aligned}
$$

so we can indeed use the standard algorithm.

Note that the above algorithm is easily modified to solve the Lyapunov equation (2.17) for the matrix $V_{j}$ for the true value function of the original problem. 


\section{A unit discount factor}

The expected discounted losses (2.3) and (2.6) are normally bounded only for $\delta<1$. More precisely, $w_{j}(j=1, \ldots, n)$ in $(2.15)$ is normally bounded only for $\delta<1$. The case $\delta=1$ can be handled by scaling the intertemporal loss function by $1-\delta$ for $\delta<1$ and then consider the limit when $\delta \rightarrow 1$, as mentioned in footnote 5. That is, we can replace the intertemporal loss function in (2.3) and (2.6) by $\mathrm{E}_{t}(1-\delta) \sum_{\tau=0}^{\infty} \delta^{\tau} L_{t+\tau}$ and $\mathrm{E}_{t}(1-\delta) \sum_{\tau=0}^{\infty} \delta^{\tau} \tilde{L}_{t+\tau}$, respectively. In particular, we can write (2.15) as

$$
(1-\delta) \tilde{X}_{t}^{\prime} V_{j_{t}} \tilde{X}_{t}+\delta w_{j} \equiv \min \mathrm{E}_{t}(1-\delta) \sum_{\tau=0}^{\infty} \delta^{\tau} L_{t+\tau}
$$

Then, $V_{j}(j=1, \ldots, n)$ still satisfies $(2.17)$, whereas $w_{j}$ now satisfies

$$
w_{j}(\delta)=\sum_{k} P_{j k}\left\{(1-\delta) \operatorname{tr}\left[V_{k}(\delta) \tilde{C}_{k} \tilde{C}_{k}^{\prime}\right]+\delta w_{k}(\delta)\right\} \quad(j=1, \ldots, n),
$$

where our notation emphasizes that $w_{j}$ and $V_{j}$ depend on $\delta$.

From (C.1), we see that

$$
\lim _{\delta \rightarrow 1-} \min \mathrm{E}_{t}(1-\delta) \sum_{\tau=0}^{\infty} \delta^{\tau} L_{t+\tau}=w_{j}(1) \quad(j=1, \ldots, n) .
$$

Furthermore, from (C.2), we see that

$$
w_{j}(1)=\sum_{k} P_{j k} w_{k}(1) \quad(j=1, \ldots, n),
$$

so the vector $\left[w_{1}(1), \ldots, w_{n}(1)\right]^{\prime}$ is an eigenvector for the eigenvalue 1 of the transition matrix $P$. By our assumptions on the Markov chain in footnote 6, the Markov chain is fully regular, so the only such eigenvector is $(1, \ldots, 1)$ (and scalar multiples thereof) (Gantmacher [16]). Therefore, $w_{j}(1)$ is independent of $j$ :

$$
w_{j}(1)=w \quad(j=1, \ldots, n)
$$

for some scalar $w$.

For $\delta<1$, we multiply (C.2) by $\bar{p}_{j}$ and sum over $j$. This gives

$\sum_{j} \bar{p}_{j} w_{j}(\delta)=\sum_{j} \sum_{k} \bar{p}_{j} P_{j k}\left\{(1-\delta) \operatorname{tr}\left[V_{k}(\delta) \tilde{C}_{k} \tilde{C}_{k}^{\prime}\right]+\delta w_{k}\right\}=(1-\delta) \sum_{k} \bar{p}_{k} \operatorname{tr}\left[V_{k}(\delta) \tilde{C}_{k} \tilde{C}_{k}^{\prime}\right]+\delta \sum_{k} \bar{p}_{k} w_{k}(\delta)$.

Letting $\bar{w}(\delta) \equiv \sum_{j} \bar{p}_{j} w_{j}(\delta)$, we see that

$$
\bar{w}(\delta)=\sum_{k} \bar{p}_{k} \operatorname{tr}\left[V_{k}(\delta) \tilde{C}_{k} \tilde{C}_{k}^{\prime}\right]
$$


We conclude that in the limit, when $\delta \rightarrow 1$, the expected minimum loss is given by

$$
w_{j}(1)=\bar{w}(1)=\sum_{k} \bar{p}_{k} \operatorname{tr}\left[V_{k}(1) \tilde{C}_{k} \tilde{C}_{k}^{\prime}\right] \quad(j=1, \ldots, n)
$$

and is independent of $\tilde{X}_{t}$ and $j_{t}$. Intuitively, for $\delta \rightarrow 1$, current losses become insignificant relative to expected losses far into the future, and then the stationary distribution $\bar{p}$ applies. Therefore, the expected discounted loss becomes independent of both the current predetermined variables and the current mode, even though the optimal policy function depends on the current mode (when the modes are observable) or the distribution of the current modes (when the modes are unobservable, as in section 7 and appendix I).

\section{Mean square stability}

Costa, Fragoso, and Marques [11, chapter 3] (CFM) provide a discussion of stability for MJLQ systems. An appropriate concept of stability for our purpose is mean square stability, which is defined as follows:

Consider the system

$$
X_{t+1}=\Gamma_{\theta_{t}} X_{t}
$$

for $t=0,1, \ldots$, where $X_{t} \in R^{n_{X}}, \theta_{t} \in \Theta \equiv\{1, \ldots, N\}$ is a Markov process with transition probabilities $\mathcal{P}_{j k}=\operatorname{Pr}\left\{\theta_{t+1}=k \mid \theta_{t}=j\right\}(j, k \in \Theta)$, transition matrix $\mathcal{P}=\left[\mathcal{P}_{j k}\right]$, and $\Gamma_{\theta}$ is an $n_{X} \times n_{X}$ matrix that depends on $\theta \in \Theta$, and $X_{0} \in R^{n_{X}}$ and $\theta_{0} \in \Theta$ are given. The system is mean square stable (MSS) if, for any initial $X_{0} \in R^{n_{X}}$ and $\theta_{0} \in \Theta$, there exist a vector $\mu \in R^{n_{X}}$ and an $n_{X} \times n_{X}$ matrix $Q$ independent of $x_{0}$ and $\theta_{0}$ such that $\left\|\mathrm{E}\left[X_{t}\right]-\mu\right\| \rightarrow 0$ and $\left\|\mathrm{E}\left[X_{t} X_{t}^{\prime}\right]-Q\right\| \rightarrow 0$ when $t \rightarrow \infty$.

CFM [11, theorem 3.9] provide six equivalent necessary and sufficient conditions for mean square stability. The following necessary and sufficient condition is appropriate for our purpose:

Define the matrices $\mathcal{C}$ and $\mathcal{N}$ by

$$
\begin{gathered}
\mathcal{C} \equiv \mathcal{P}^{\prime} \otimes I_{n_{X}^{2}}, \\
\mathcal{N} \equiv \operatorname{diag}\left(\Gamma_{\theta} \otimes \Gamma_{\theta}\right) \equiv\left[\begin{array}{cccc}
\Gamma_{1} \otimes \Gamma_{1} & 0 & \cdots & 0 \\
0 & \Gamma_{2} \otimes \Gamma_{2} & \ddots & \vdots \\
\vdots & \ddots & \ddots & 0 \\
0 & \cdots & 0 & \Gamma_{N} \otimes \Gamma_{N}
\end{array}\right] .
\end{gathered}
$$

The system above is MSS if and only if the spectral radius (the supremum of the modulus of the eigenvalues) of the matrix $\mathcal{C N}$ is less than unity. 
Applying CFM's definition of and conditions for mean square stability requires a simple redefinition of the modes in our framework. Start from the system

$$
X_{t+1}=M_{j_{t} j_{t+1}} X_{t}
$$

where $t=0,1, \ldots, X_{t} \in R^{n_{X}}, j_{t} \in\{1, \ldots, n\}, P=\left[P_{j k}\right], P_{j k}=\operatorname{Pr}\left\{j_{t+1}=k \mid j_{t}=j\right\}$, and $X_{0}$ and $j_{0}$ are given. This system differs from CFM's system in that the matrix $M_{j_{t} j_{t+1}}$ depends on the realization of the modes in both period $t$ and period $t+1$.

Define the new composite mode $\theta_{t} \equiv\left(j_{t}, j_{t+1}\right)$, which can take $N=n^{2}$ values, and consider a Markov chain for $\theta_{t}$ with transition probabilities $\mathcal{P}_{\theta \kappa} \equiv \operatorname{Pr}\left\{\theta_{t+1}=\kappa \equiv(k, l) \mid \theta_{t}=\theta \equiv(j, k)\right\}$. We note that the transition probability from $\theta_{t}=(j, k)$ to $\theta_{t+1}=(k, l)$ does not depend on $j$ but only on $k$ and $l$. Furthermore, it is simply $P_{k l}$, so

$$
\mathcal{P}_{(j, k),(k, l)}=P_{k l} \quad(j, k, l=1, \ldots, n) .
$$

Thus, we can consider the new system

$$
X_{t+1}=M_{\theta_{t}} X_{t}
$$

where $\theta_{t}$ is a Markov chain that can take $n^{2}$ different values and has a transition matrix $\mathcal{P}$ with the transition probabilities $\mathcal{P}_{\theta_{t} \theta_{t+1}}$ defined above. Then the results of CFM on MSS apply directly, and we only need to define $\Gamma_{\theta}, \mathcal{P}, \mathcal{C}$, and $\mathcal{N}$ using the $n^{2}$-mode composite Markov chain for $\theta_{t} \equiv\left(j_{t}, j_{t+1}\right)$ instead of just the $n$-mode chain for $j_{t}$.

\section{E Alternative models with different predetermined and forward- looking variables}

Our MJLQ framework allows us to consider situations when the modes $j=1, \ldots, n$ correspond to alternative structural models, including not only when some coefficients are zero or nonzero but also when a variable is predetermined in one model and forward-looking in another. This allows us include optimal policy when it is known what structural model is true in the current period but there is uncertainty about the true structural model in the future. ${ }^{21}$

\footnotetext{
21 If the current model is not observed, we would have to include Bayesian learning of the subjective probability distribution over models and encounter problems of experimentation versus "adaptive" loss minimization [give reference(s)].
} 
In order to see this, consider a particular simple example, when there are two modes, $j=1,2$, with transition matrix $P=\left[P_{j k}\right], j, k=1,2$. Let $j=1$ corresponds to a model with an acceleration Phillips curve (the AP model),

$$
\pi_{t+1}=\pi_{t}+\alpha y_{t}+\varepsilon_{1, t+1}
$$

and let $j=2$ corresponds to a New Keynesian Phillips curve (the NK model),

$$
\mathrm{E}_{t} \pi_{t+1}=\pi_{t}-\kappa y_{t}-\varepsilon_{2, t}
$$

where $\varepsilon_{1 t}$ and $\varepsilon_{2 t}$ are i.i.d. with zero means. Thus, inflation, $\pi_{t+1}$ is predetermined in AP model and forward-looking in the NK model. Regard the output gap, $y_{t}$, as the control variable, for simplicity.

Let $\pi_{t}$ denote actual inflation in period $t$, and introduce the two variables $\pi_{1 t}$ and $\pi_{2 t}$, where $\pi_{1 t}$ is predetermined and denotes inflation in the AP model (AP inflation) and $\pi_{2 t}$ is forward-looking and denotes inflation in the NK model (NK inflation). Actual actual inflation then satisfies

$$
\pi_{t}=\theta_{t} \pi_{1 t}+\left(1-\theta_{t}\right) \pi_{2 t}
$$

where $\theta_{t}=1$ in mode 1 and $\theta_{t}=0$ in mode 2 . We thus have

$$
\begin{aligned}
\pi_{1, t+1} & =\pi_{t}+\alpha y_{t}+\varepsilon_{1, t+1}, \\
\mathrm{E}_{t} \pi_{t+1} & =\pi_{2 t}-\kappa y_{t}-\varepsilon_{2 t},
\end{aligned}
$$

where we assume that, in the AP model, current actual inflation affects future AP inflation and, in the NK model, the expected future actual inflation affects current NK inflation.

We want to write this model as (2.1) and (2.2) by suitable definitions of $X_{t}, x_{t}, i_{t}$, and $\varepsilon_{t}$, and the matrices. The trick is to treat actual inflation, $\pi_{t}$, as a non-predetermined variable even though this is not the case when the AP model is true. This works, because an additional predetermined variable identical to an existing predetermined variable can always be introduced as a trivial nonpredetermined variable by adding an equation in the block of equations for the forward-looking variables. Suppose that the new variable, $y_{t}$, is identical to an existing predetermined variable, $X_{1 t}$, say. Then we can just add the equation

$$
0=X_{1 t}-y_{t}
$$

to that block, where the left side has zero instead of a linear combination of expected future forwardlooking variables. Generally, a new variable that is a linear combination of current predetermined 
and forward-looking variables can always be introduced as a new forward-looking variable in this way.

Observe that

$$
\begin{aligned}
\mathrm{E}_{t} \pi_{t+1} & =\mathrm{E}_{t}\left[\theta_{t+1} \pi_{1, t+1}+\left(1-\theta_{t+1}\right) \pi_{2, t+1}\right] \\
& =\mathrm{E}_{t} \theta_{t+1}\left(\pi_{t}+\alpha y_{t}\right)+\mathrm{E}_{t}\left(1-\theta_{t+1}\right) \pi_{2, t+1}
\end{aligned}
$$

and use this to substitute for $\mathrm{E}_{t} \pi_{t+1}$ in (E.1). Let $X_{t} \equiv\left(\pi_{1 t}, \varepsilon_{2 t}\right)^{\prime}, x_{t} \equiv\left(\pi_{2 t}, \pi_{t}\right)^{\prime}$, and $i_{t} \equiv y_{t}$. Then we can write the model in the form (2.1) and (2.2) as

$$
\begin{aligned}
X_{t+1} & =\left[\begin{array}{ll}
1 & 0 \\
0 & 0
\end{array}\right] X_{t}+\left[\begin{array}{ll}
0 & 0 \\
0 & 0
\end{array}\right] x_{t}+\left[\begin{array}{l}
\alpha \\
0
\end{array}\right] i_{t}+\left[\begin{array}{c}
\varepsilon_{1, t+1} \\
\varepsilon_{2, t+1}
\end{array}\right] \\
\mathrm{E}_{t}\left[\begin{array}{cc}
1-\theta_{t+1} & 0 \\
0 & 0
\end{array}\right] x_{t+1} & =\left[\begin{array}{cc}
0 & -1 \\
\theta_{t} & 0
\end{array}\right] X_{t}+\left[\begin{array}{cc}
0 & 1-\mathrm{E}_{t} \theta_{t+1} \\
1-\theta_{t} & -1
\end{array}\right] x_{t}+\left[\begin{array}{c}
-\kappa-\alpha \mathrm{E}_{t} \theta_{t+1} \\
0
\end{array}\right] i_{t} .
\end{aligned}
$$

\section{F Details of the estimation}

Here we lay out the details of the priors we use in our Bayesian estimation.

For the RS model in section 4.1, we base our prior for the MJLQ case on our OLS estimates. The priors are identical across modes. In particular, the priors for the vectors of coefficients $\left[\alpha_{i}\right]$ and $\left[\beta_{i}\right]$ are each multivariate normal distributions, with mean given by the OLS point estimates and a covariance matrix given by the covariance matrix of the estimates scaled up by a factor of 4. For the parameters of the transition matrix $P$ of the Markov chain, we take independent beta distributions (subject to the constraint that the rows sum to one). We let the diagonal elements have mean 0.9 and standard deviation 0.08 , while the off-diagonals have means 0.05 and standard deviations 0.05. For the variances of the shocks, we assume an inverse gamma prior distribution with two degrees of freedom.

For the Lindé model in section 4.2, we take independent priors for the different structural coefficients, again with the priors being identical across modes. For the coefficients $\omega_{f}$ and $\beta_{f}$, we assume a beta distribution with mean 0.5 and standard deviation 0.25 . The other structural coefficients have normal distributions, with $\gamma \sim N(0.1,0.05), \beta_{r} \sim N(0.15,0.075), \beta_{y} \sim N(1.5,0.5)$,

$\rho_{1} \sim N(0.9,0.2), \rho_{1} \sim N(0.2,0.2), \gamma_{\pi} \sim N(1.5,0.5)$, and $\gamma_{y} \sim N(0.5,0.5)$. Again for the variances of the shocks, we assume an inverse gamma prior distribution with two degrees of freedom. The prior over the Markov chain transition matrix is the same as in the RS model. 


\section{G Details for arbitrary time-varying instrument rules}

For $t=0, \ldots, T-1$, introduce the new $\left(n_{\tilde{\imath}}+n_{i}\right) \times 1$ vector of instruments,

$$
\hat{\imath}_{t} \equiv\left[\begin{array}{c}
\tilde{\imath}_{t} \\
\varphi_{t}
\end{array}\right]
$$

and write the model

$$
\tilde{X}_{t+1}=\tilde{A}_{j_{t+1}} \tilde{X}_{t}+\hat{B}_{j_{t+1}} \hat{\imath}_{t}+\tilde{C}_{j_{t+1}} \varepsilon_{t+1}
$$

where the new $n_{\tilde{X}} \times\left(n_{\tilde{\imath}}+n_{i}\right)$ matrix $\hat{B}_{j_{t+1}}$ satisfies

$$
\hat{B}_{j_{t+1}} \equiv\left[\begin{array}{ll}
\tilde{B}_{j_{t+1}} & 0_{n_{\tilde{X}} \times n_{i}}
\end{array}\right]
$$

Partition the $\left(n_{\tilde{X}}+n_{\tilde{\imath}}\right) \times\left(n_{\tilde{X}}+n_{\tilde{\imath}}\right)$ matrix $\tilde{W}_{j_{t}}$ conformably with $\tilde{X}_{t}$ and $\tilde{\imath}_{t}$ as

$$
\tilde{W}_{j_{t}}=\left[\begin{array}{cc}
Q_{j_{t}} & N_{j_{t}} \\
N_{j_{t}}^{\prime} & R_{j_{t}}
\end{array}\right]
$$

Furthermore, write the augmented period loss as

$$
\hat{L}_{t} \equiv\left[\begin{array}{c}
\tilde{X}_{t} \\
\hat{\imath}_{t}
\end{array}\right]^{\prime}\left[\begin{array}{cc}
Q_{j_{t}} & \hat{N}_{t j_{t}} \\
\hat{N}_{t j_{t}}^{\prime} & \hat{R}_{t j_{t}}
\end{array}\right]\left[\begin{array}{c}
\tilde{X}_{t} \\
\hat{\imath}_{t}
\end{array}\right]
$$

where the new $n_{\tilde{X}} \times\left(n_{\tilde{\imath}}+n_{x}\right)$ and $\left(n_{\tilde{\imath}}+n_{x}\right) \times\left(n_{\tilde{\imath}}+n_{x}\right)$ matrices $\hat{N}_{j_{t}}$ and $\hat{R}_{j_{t}}$ satisfy, respectively,

$$
\hat{N}_{t j_{t}} \equiv\left[\begin{array}{cc}
N_{j_{t}} & -F_{\tilde{X} t j_{t}}^{\prime} / 2
\end{array}\right], \quad \hat{R}_{t j_{t}} \equiv\left[\begin{array}{cc}
R_{j_{t}} & -F_{\tilde{\imath} t j_{t}}^{\prime} / 2 \\
-F_{\tilde{\imath} t j_{t}} / 2 & 0_{n_{\tilde{\imath}} \times n_{\tilde{\imath}}}
\end{array}\right] .
$$

Then, the first-order condition for an optimum of the Bellman equation will, in the standard way, result in a time- and mode-dependent optimal policy function

$$
\hat{\imath}_{t}=\hat{F}_{t j_{t}} \tilde{X}_{t} \quad\left(0 \leq t \leq T-1,0 \leq j_{t} \leq n\right)
$$

which is defined in a compact way as

$$
\hat{F}_{t j_{t}} \equiv-J_{t j_{t}}^{-1} K_{t j_{t}}
$$

where $J_{t j_{t}}$ and $K_{t j_{t}}$ are defined recursively from $\tilde{V}_{t+1, j_{t}}$ as

$$
\begin{aligned}
J_{t j_{t}} & \equiv \hat{R}_{t j_{t}}+\delta \mathrm{E}_{t} \hat{B}_{j_{t+1}}^{\prime} \tilde{V}_{t+1, j_{t+1}} \hat{B}_{j_{t+1}}=\hat{R}_{t j_{t}}+\delta \sum_{k} P_{j_{t} k} \hat{B}_{k}^{\prime} \tilde{V}_{t+1, k} \hat{B}_{k} \\
K_{t j_{t}} & \equiv \hat{N}_{t j_{t}}^{\prime}+\delta \mathrm{E}_{t} \hat{B}_{j_{t+1}}^{\prime} \tilde{V}_{t+1, j_{t+1}} \tilde{A}_{j_{t+1}}=\hat{N}_{t j_{t}}^{\prime}+\delta \sum_{k} P_{j_{t} k} \hat{B}_{k}^{\prime} \tilde{V}_{t+1, k} \tilde{A}_{k}
\end{aligned}
$$


Substitution of this optimal policy function in the Bellman equation results in the recursive equation for $\tilde{V}_{t j_{t}}$,

$$
\tilde{V}_{t j_{t}}=Q_{j_{t}}+\delta \mathrm{E}_{t} \tilde{A}_{j_{t+1}}^{\prime} \tilde{V}_{t+1, j_{t+1}} \tilde{A}_{j_{t+1}}-K_{t j_{t}}^{\prime} J_{t j_{t}}^{-1} K_{t j_{t}}=Q_{j_{t}}+\delta \sum_{k} P_{j_{t} k} \tilde{A}_{k}^{\prime} \tilde{V}_{t+1, k} \tilde{A}_{k}-K_{t j_{t}}^{\prime} J_{t j_{t}}^{-1} K_{t j_{t}}
$$

Finally, the optimal policy function $\tilde{F}_{t j_{t}}$ for $t=0, \ldots, T-1$ can be identified by partitioning $\hat{F}_{t j_{t}}$ conformably with $\tilde{\imath}_{t}$ and $\varphi_{t}$,

$$
\hat{F}_{t j_{t}} \equiv\left[\begin{array}{c}
\tilde{F}_{t j_{t}} \\
F_{\varphi t j_{t}}
\end{array}\right]
$$

\section{H Details for arbitrary time-invariant instrument rules}

Consider the case when the time-invariant instrument rule can be written

$$
i_{t}=F_{X j_{t}} X_{t}+F_{x j_{t}} x_{t} \quad\left(j_{t}=1, \ldots, n\right)
$$

and the instrument rate hence does not respond to $\Xi_{t-1}$. In that case, we can use a simpler algorithm than letting $t \rightarrow-\infty$ in the algorithm described in appendix G. If there is a unique solution associated with a specified instrument rule, it will determine the forward-looking variables as a linear function of the predetermined variables,

$$
x_{t}=G_{j_{t}} X_{t}
$$

Given a quadratic intertemporal loss function, this will also determine a value of the loss function of the form

$$
X_{t}^{\prime} V_{j_{t}} X_{t}+w_{j_{t}}
$$

In order to specify an algorithm for finding $G_{j}, V_{j}$, and $w_{j}$, suppose the instrument rule can be written as (H.1). Consider period $t+1$, and assume that $G_{j_{t+1}}^{(t+1)}$ in

$$
x_{t+1}=G_{j_{t+1}}^{(t+1)} X_{t+1} \quad\left(j_{t+1}=1, \ldots, n\right),
$$

is known in period $t$. This will imply

$$
\begin{aligned}
\mathrm{E}_{t} H_{j_{t+1}} x_{t+1} & =\mathrm{E}_{t} H_{j_{t+1}} G_{j_{t+1}}^{(t+1)} X_{t+1} \\
& =\sum_{k} P_{j k} H_{k} G_{k}^{(t+1)}\left[\left(A_{11 k}+B_{1 k} F_{X j}\right) X_{t}+\left(A_{12 k}+B_{1 k} F_{x j}\right) x_{t}\right] \\
& =\left(A_{21 j}+B_{2 j} F_{X j}\right) X_{t}+\left(A_{22 j}+B_{2 j} F_{x j}\right) x_{t} .
\end{aligned}
$$


We can then solve for $x_{t}$ in period $t$,

$$
x_{t}=G_{j}^{(t)} X_{t}
$$

where

$$
\begin{aligned}
G_{j}^{(t)} \equiv & {\left[A_{22 j}+B_{2 j} F_{x j}-\sum_{k} P_{j k} H_{k} G_{k}^{(t+1)}\left(A_{12 k}+B_{1 k} F_{x j}\right)\right]^{-1} } \\
& \cdot\left[\sum_{k} P_{j k} H_{k} G_{k}^{(t+1)}\left(A_{11 k}+B_{1 k} F_{X j}\right)-\left(A_{21 j}+B_{2 j} F_{X j}\right)\right] .
\end{aligned}
$$

It follows that, starting with a guess $G_{j}^{0}$, the iteration for $l=0,1, \ldots$, according to

$$
\begin{aligned}
G_{j}^{l+1}= & {\left[A_{22 j}+B_{2 j} F_{x j}-\sum_{k} P_{j k} H_{k} G_{k}^{l}\left(A_{12 k}+B_{1 k} F_{x j}\right)\right]^{-1} } \\
& \cdot\left[\sum_{k} P_{j k} H_{k} G_{k}^{l}\left(A_{11 k}+B_{1 k} F_{X j}\right)-\left(A_{21 j}+B_{2 j} F_{X j}\right)\right],
\end{aligned}
$$

will hopefully make $G_{j}^{l}$ converge to the correct $G_{j}$,

$$
x_{t}=G_{j} X_{t}
$$

This then implies

$$
X_{t+1}=M_{j k} X_{t}+C_{k} \varepsilon_{t+1}
$$

where

$$
M_{j k} \equiv A_{11 k}+A_{12 k} G_{j}+B_{1 k}\left(F_{X j}+F_{x j} G_{j}\right)
$$

Clearly, $G \equiv\left\{G_{j}\right\}$ and $M \equiv\left\{M_{j k}\right\}$ will be functions of $F \equiv\left\{\left(F_{X j}, F_{x j}\right)\right\}$.

Let the period loss function be

$$
L_{t}=\left[\left[\begin{array}{c}
X_{t} \\
x_{t} \\
i_{t}
\end{array}\right]\right]^{\prime}\left[\begin{array}{cc}
Q & N \\
N^{\prime} & R
\end{array}\right]\left[\left[\begin{array}{c}
X_{t} \\
x_{t} \\
i_{t}
\end{array}\right]\right]
$$

Given (H.1), (H.2), and (H.3), we can define the matrix

$$
\bar{W}_{j} \equiv\left[\begin{array}{c}
{\left[\begin{array}{c}
I \\
G_{j}
\end{array}\right]} \\
F_{X j}+F_{x j} G_{j}
\end{array}\right]^{\prime}\left[\begin{array}{cc}
Q & N \\
N^{\prime} & R
\end{array}\right]\left[\begin{array}{c}
I \\
G_{j} \\
F_{X j}+F_{x j} G_{j}
\end{array}\right],
$$

in which case the period loss satisfies

$$
L_{t}=X_{t}^{\prime} \bar{W}_{j} X_{t}
$$


It follows that the value function corresponding to the intertemporal loss function

$$
\mathrm{E}_{t} \sum_{\tau=0}^{\infty} \delta^{\tau} L_{t+\tau}
$$

will satisfy

$$
X_{t}^{\prime} V_{j} X_{t}+w_{j}=X_{t}^{\prime} \bar{W}_{j} X_{t}+\delta \sum_{k} P_{j k}\left[X_{t}^{\prime} M_{j k}^{\prime} V_{k} M_{j k} X_{t}+\operatorname{tr}\left(V_{k} C_{k} C_{k}^{\prime}\right)+w_{k}\right]
$$

Hence, the matrix $V_{j}$ will satisfy the Lyapunov equation

$$
V_{j}=\bar{W}_{j}+\delta \sum_{k} P_{j k} M_{j k}^{\prime} V_{k} M_{j k} \quad(j=1, \ldots, n)
$$

and $w_{j}$ will satisfy

$$
w_{j}=\delta \sum_{k} P_{j k}\left[\operatorname{tr}\left(V_{k} C_{k} C_{k}^{\prime}\right)+w_{k}\right] \quad(j=1, \ldots, n) .
$$

Note that we can, for each $j$, define

$$
\begin{aligned}
\hat{W}_{j} & \equiv \bar{W}_{j}+\delta \sum_{k \neq j} P_{j k} M_{j k}^{\prime} V_{k} M_{j k} \\
\hat{M}_{j j} & =\sqrt{\delta P_{j j}} M_{j j}
\end{aligned}
$$

and then solve the more standard Lyapunov equation

$$
V_{j}=\hat{W}_{j}+\hat{M}_{j j}^{\prime} V_{j} \hat{M}_{j j} \quad(j=1, \ldots, n) .
$$

Clearly, $V \equiv\left\{V_{j}\right\}$ will be a function of $F$ and $\delta$, and $w \equiv\left\{w_{j}\right\}$ will be a function of $F, \delta$ and $\Sigma$.

Let $\bar{p}_{j}(j=1, \ldots, n)$ denote the stationary distribution of the states, and let $\bar{V} \equiv \sum_{j} \bar{p}_{j} V_{j}$ and $\bar{w} \equiv \sum_{j} \bar{p}_{j} w_{j}$ denote the unconditional means of $V_{j}$ and $w_{j}$. We note that

$$
\bar{w}=\frac{\delta}{1-\delta} \sum_{k} \bar{p}_{k} \operatorname{tr}\left(V_{k} C_{k} C_{k}^{\prime}\right) .
$$

Suppose that the intertemporal loss function is $1-\delta$ times the one above,

$$
\mathrm{E}_{t} \sum_{\tau=0}^{\infty}(1-\delta) \delta^{\tau} L_{t+\tau},
$$

and suppose that we consider the limit when $\delta \rightarrow 1$,

$$
\lim _{\delta \rightarrow 1} \mathrm{E}_{t} \sum_{\tau=0}^{\infty}(1-\delta) \delta^{\tau} L_{t+\tau}=\mathrm{E}\left[L_{t}\right] .
$$


In that case, the intertemporal loss function is just the unconditional mean of the period loss function, $\mathrm{E}\left[L_{t}\right]$. Furthermore, the unconditional mean of $1-\delta$ times the value function above will be

$$
(1-\delta)\left\{\mathrm{E}\left[X_{t}^{\prime} V_{j_{t}} X_{t}\right]+\bar{w}\right\}=(1-\delta) \mathrm{E}\left[X_{t}^{\prime} V_{t} X_{t}\right]+\delta \sum_{k} \bar{p}_{k} \operatorname{tr}\left(V_{k} C_{k} C_{k}^{\prime}\right)
$$

We see that, when $\delta \rightarrow 1$, the first term on the right side goes to zero, and we conclude that, in the limit,

$$
\mathrm{E}\left[L_{t}\right]=\sum_{k} \bar{p}_{k} \operatorname{tr}\left[V_{k}(F, 1) C_{k} C_{k}^{\prime}\right]
$$

where we also explicitly note that $V_{k}$ depends on $F$ and $\delta$.

Suppose the instrument rule is restricted to a given class $\mathcal{F}$ of instrument rules

$$
F \in \mathcal{F}
$$

The optimal instrument rule in this class, $\hat{F}$, can now be defined as

$$
\hat{F} \equiv \arg \min _{F \in \mathcal{F}} \sum_{k} \bar{p}_{k} \operatorname{tr}\left[V_{k}(F, 1) C_{k} C_{k}^{\prime}\right]
$$

It will obviously depend on $C_{k} C_{k}^{\prime}$, the covariance matrix of the shock $C_{k} \varepsilon_{t+1}$. Hence, certainty equivalence does generally not hold for optimal restricted instrument rules.

\section{Details with unobservable modes}

\section{I.1 Unobservable modes and forward-looking variables}

Consider the dual saddlepoint problem with $\tilde{X}_{t}$ given, unobservable modes, and the distribution $p_{t}$ of modes in period $t$. For notational convenience, it is practical to change the order of variables in the dual instrument vector, put the instrument first, and denote it by $\hat{\imath}_{t j}$,

$$
\hat{\imath}_{t j} \equiv\left[\begin{array}{c}
i_{t} \\
x_{t j} \\
\gamma_{t j}
\end{array}\right] \text {. }
$$

We note that $i_{t}$ will only depend on $p_{t}$ and be independent of $j$, whereas $x_{t j}$ and $\gamma_{t j}$ will depend

on both $p_{t}$ and $j$. Instead of the dual matrix $\tilde{W}_{j}$, we then define the dual matrix $\hat{W}_{j}$ accordingly and partition it conformably with $\tilde{X}_{t}$ and $\hat{\imath}_{t j}$ as

$$
\hat{W}_{j} \equiv\left[\begin{array}{cc}
Q_{j} & \hat{N}_{j} \\
\hat{N}_{j}^{\prime} & \hat{R}_{j}
\end{array}\right] .
$$


The value function for the dual problem will be quadratic in $\tilde{X}_{t}$ and can be written

$$
\tilde{X}_{t}^{\prime} \tilde{V}\left(p_{t}\right) \tilde{X}_{t}+\tilde{w}\left(p_{t}\right)
$$

where $\tilde{V}\left(p_{t}\right)$ is a symmetric positive semidefinite matrix and $\tilde{w}\left(p_{t}\right)$ is a scalar. It will satisfy the Bellman equation

$$
\tilde{X}_{t}^{\prime} \tilde{V}\left(p_{t}\right) \tilde{X}_{t}+\tilde{w}\left(p_{t}\right)=\min _{i_{t}} \sum_{j} p_{t j} \max _{\gamma_{t j}} \min _{x_{t j}}\left\{\begin{array}{l}
\tilde{X}_{t}^{\prime} Q_{j} \tilde{X}_{t}+2 \tilde{X}_{t}^{\prime} \hat{N}_{j} \hat{\imath}_{t j}+\hat{\imath}_{t j}^{\prime} \hat{R}_{j} \hat{\imath}_{t j} \\
+\delta \sum_{k} P_{j k}\left[\tilde{X}_{t+1, k}^{\prime} \tilde{V}\left(p_{t} P\right) \tilde{X}_{t+1, k}+\tilde{w}\left(p_{t} P\right)\right]
\end{array}\right\}
$$

where

$$
\tilde{X}_{t+1, k}=\tilde{A}_{k} \tilde{X}_{t}+\hat{B}_{k} \hat{\imath}_{t j}+\tilde{C}_{k} \varepsilon_{t+1}
$$

and the matrix $\hat{B}_{k}$ is used instead of $\tilde{B}_{k}$ and has columns ordered according to $\hat{\imath}_{t j}$.

The first-order conditions with respect to $i_{t}$ and $\tilde{x}_{t j} \equiv\left(x_{t j}^{\prime}, \gamma_{t j}^{\prime}\right)^{\prime}$ are, respectively,

$$
\begin{gathered}
\sum_{j} p_{t j}\left[\tilde{X}_{t}^{\prime} \hat{N}_{\cdot i j}+\hat{\imath}_{t j}^{\prime} \hat{R}_{\cdot i j}+\delta \sum_{k} P_{j k}\left(\tilde{X}_{t}^{\prime} \tilde{A}_{k}^{\prime}+\hat{\imath}_{t j}^{\prime} \hat{B}_{k}^{\prime}\right) \tilde{V}\left(p_{t} P\right) \hat{B}_{\cdot i k}\right]=0, \\
\tilde{X}_{t}^{\prime} \hat{N}_{\cdot \tilde{x} j}+\hat{\imath}_{t j}^{\prime} \hat{R}_{\cdot \tilde{x} j}+\delta \sum_{k} P_{j k}\left(\tilde{X}_{t}^{\prime} \tilde{A}_{k}^{\prime}+\hat{\imath}_{t j}^{\prime} \hat{B}_{k}^{\prime}\right) \tilde{V}\left(p_{t} P\right) \hat{B}_{\cdot \tilde{x} k}=0 \quad(j=1, \ldots, n),
\end{gathered}
$$

where $\hat{N}_{j}, \hat{R}_{j}$ and $\hat{B}_{k}$ are partitioned conformably with $i_{t}$ and $\tilde{x}_{t j}$ as

$$
\hat{N}_{j} \equiv\left[\begin{array}{ll}
\hat{N}_{\cdot i j} & \hat{N}_{\cdot \tilde{x} j}
\end{array}\right], \quad \hat{R}_{j} \equiv\left[\begin{array}{ll}
\hat{R}_{\cdot i j} & \hat{R}_{\cdot \tilde{x} j}
\end{array}\right] \equiv\left[\begin{array}{cc}
\hat{R}_{i i j} & \hat{R}_{i \tilde{x} j} \\
\hat{R}_{\tilde{x} i j} & \hat{R}_{\tilde{x} \tilde{x} j}
\end{array}\right], \quad \hat{B}_{k} \equiv\left[\begin{array}{ll}
\hat{B}_{\cdot i k} & \hat{B}_{\cdot \tilde{x} k}
\end{array}\right] .
$$

We can rewrite the first-order conditions as

$$
\begin{gathered}
\sum_{j} p_{t j}\left[\hat{N}_{\cdot i j}^{\prime} \tilde{X}_{t}+\hat{R}_{i i j} i_{t}+\hat{R}_{i \tilde{x} j} \tilde{x}_{t j}+\delta \sum_{k} P_{j k} \hat{B}_{\cdot i k}^{\prime} \tilde{V}\left(p_{t} P\right)\left(\tilde{A}_{k} \tilde{X}_{t}+\hat{B}_{\cdot i k} i_{t}+\hat{B}_{\cdot \tilde{x} k} \tilde{x}_{t j}\right)\right]=0, \\
\hat{N}_{\cdot \tilde{x} j}^{\prime} X_{t}+\hat{R}_{\tilde{x} i j} i_{t}+\hat{R}_{\tilde{x} \tilde{x} j} \tilde{x}_{t j}+\delta \sum_{k} P_{j k} \hat{B}_{\cdot \tilde{x} k}^{\prime} \tilde{V}\left(p_{t} P\right)\left(\tilde{A}_{k} \tilde{X}_{t}+\hat{B}_{\cdot i k} i_{t}+\hat{B}_{\cdot \tilde{x} k} \tilde{x}_{t j}\right)=0 \quad(j=1, \ldots, n) .
\end{gathered}
$$

It is then apparent that the first-order conditions can be written compactly as

$$
J\left(p_{t}\right)\left[\begin{array}{c}
i_{t} \\
\tilde{x}_{t 1} \\
\vdots \\
\tilde{x}_{t n}
\end{array}\right]+K\left(p_{t}\right) \tilde{X}_{t}=0
$$

where

$$
J\left(p_{t}\right) \equiv\left[\begin{array}{cccc}
J_{i i}\left(p_{t}\right) & J_{i 1}\left(p_{t}\right) & \cdots & J_{i n}\left(p_{t}\right) \\
J_{1 i}\left(p_{t}\right) & J_{11}\left(p_{t}\right) & 0 & 0 \\
\vdots & 0 & \ddots & 0 \\
J_{n i}\left(p_{t}\right) & 0 & 0 & J_{n n}\left(p_{t}\right)
\end{array}\right]
$$




$$
\begin{aligned}
& J_{i i}\left(p_{t}\right) \equiv \sum_{j} p_{t j}\left[\hat{R}_{i i j}+\delta \sum_{k} P_{j k} \hat{B}_{\cdot i k}^{\prime} \tilde{V}\left(p_{t} P\right) \hat{B}_{\cdot i k}\right], \\
& J_{i j}\left(p_{t}\right) \equiv p_{t j}\left[\hat{R}_{i \tilde{x} j}+\delta \sum_{k} P_{j k} \hat{B}_{\cdot i k}^{\prime} \tilde{V}\left(p_{t} P\right) \hat{B}_{\cdot \tilde{x} k}\right] \quad(j=1, \ldots, n), \\
& J_{j i}\left(p_{t}\right) \equiv \hat{R}_{\tilde{x} i j}+\delta \sum_{k} P_{j k} \hat{B}_{\cdot \tilde{x} k}^{\prime} \tilde{V}\left(p_{t} P\right) \hat{B}_{\cdot i k} \quad(j=1, \ldots, n), \\
& J_{j j}\left(p_{t}\right) \equiv \hat{R}_{\tilde{x} \tilde{x} j}+\delta \sum_{k} P_{j k} \hat{B}_{\cdot \tilde{x} k}^{\prime} \tilde{V}\left(p_{t} P\right) \hat{B}_{\cdot \tilde{x} k} \quad(j=1, \ldots, n), \\
& K\left(p_{t}\right) \equiv\left[\begin{array}{c}
\sum_{j} p_{t j}\left[\hat{N}_{\cdot i j}^{\prime}+\delta \sum_{k} P_{j k} \hat{B}_{\cdot i k}^{\prime} \tilde{V}\left(p_{t} P\right) \tilde{A}_{k}\right] \\
\hat{N}_{\cdot \tilde{x} 1}^{\prime}+\delta \sum_{k} P_{1 k} \hat{B}_{\cdot \tilde{x} k}^{\prime} \tilde{V}\left(p_{t} P\right) \tilde{A}_{k} \\
\vdots \\
\hat{N}_{\cdot \tilde{x} n}^{\prime}+\delta \sum_{k} P_{n k} \hat{B}_{\cdot \tilde{x} k}^{\prime} \tilde{V}\left(p_{t} P\right) \tilde{A}_{k}
\end{array}\right] .
\end{aligned}
$$

This leads to the optimal policy function

$$
\left[\begin{array}{c}
i_{t} \\
\tilde{x}_{t 1} \\
\vdots \\
\tilde{x}_{t n}
\end{array}\right]=\check{F}\left(p_{t}\right) \tilde{X}_{t} \equiv\left[\begin{array}{c}
F_{i}\left(p_{t}\right) \\
F_{x}\left(p_{t}\right)_{1} \\
F_{\gamma}\left(p_{t}\right)_{1} \\
\vdots \\
F_{x}\left(p_{t}\right)_{n} \\
F_{\gamma}\left(p_{t}\right)_{n}
\end{array}\right] \tilde{X}_{t}
$$

where

$$
\check{F}\left(p_{t}\right) \equiv-J\left(p_{t}\right)^{-1} K\left(p_{t}\right) .
$$

Hence, we can write

$$
\hat{\imath}_{t j} \equiv\left[\begin{array}{c}
i_{t} \\
x_{t j} \\
\gamma_{t j}
\end{array}\right]=\left[\begin{array}{c}
F_{i}\left(p_{t}\right) \\
F_{x}\left(p_{t}\right)_{j} \\
F_{\gamma}\left(p_{t}\right)_{j}
\end{array}\right] \tilde{X}_{t} \equiv \hat{F}\left(p_{t}\right)_{j} \tilde{X}_{t} \quad(j=1, \ldots, n)
$$

Furthermore, the value function for the dual saddlepoint problem satisfies

$$
\tilde{X}_{t}^{\prime} \tilde{V}\left(p_{t}\right) \tilde{X}_{t} \equiv \sum_{j} p_{t j}\left\{\begin{array}{l}
\tilde{X}_{t}^{\prime} Q_{j} \tilde{X}_{t}+2 \tilde{X}_{t}^{\prime} \hat{N}_{j} \hat{F}\left(p_{t}\right)_{j} \tilde{X}_{t}+\tilde{X}_{t}^{\prime} \hat{F}\left(p_{t}\right)_{j}^{\prime} \hat{R}_{j} \hat{F}\left(p_{t}\right)_{j} \tilde{X}_{t} \\
+\delta \sum_{k} P_{j k} \tilde{X}_{t}^{\prime}\left[\tilde{A}_{k}^{\prime}+\hat{F}\left(p_{t}\right)_{j}^{\prime} \hat{B}_{k}^{\prime}\right] \tilde{V}\left(p_{t} P\right)\left[\tilde{A}_{k}+\hat{B} k \hat{F}\left(p_{t}\right)_{j}\right] \tilde{X}_{t}
\end{array}\right\} .
$$

This implies the following Riccati equation for $\tilde{V}\left(p_{t}\right)$ :

$$
\tilde{V}\left(p_{t}\right)=\sum_{j} p_{t j}\left\{\begin{array}{l}
Q_{j}+\hat{N}_{j} \hat{F}\left(p_{t}\right)_{j}+\hat{F}\left(p_{t}\right)_{j}^{\prime} \hat{N}_{j}^{\prime}+\hat{F}\left(p_{t}\right)_{j}^{\prime} \hat{R}_{j} \hat{F}\left(p_{t}\right)_{j} \\
+\delta \sum_{k} P_{j k}\left[\tilde{A}_{k}^{\prime}+\hat{F}\left(p_{t}\right)_{j}^{\prime} \hat{B}_{k}^{\prime}\right] \tilde{V}\left(p_{t} P\right)\left[\tilde{A}_{k}+\hat{B}_{k} \hat{F}\left(p_{t}\right)_{j}\right]
\end{array}\right\} .
$$

In terms of our standard dual instrument vector, $\tilde{\imath}_{t j}$, the policy function is

$$
\tilde{\imath}_{t j} \equiv\left[\begin{array}{c}
x_{t j} \\
i_{t} \\
\gamma_{t j}
\end{array}\right]=\left[\begin{array}{c}
F_{x}\left(p_{t}\right)_{j} \\
F_{i}\left(p_{t}\right) \\
F_{\gamma}\left(p_{t}\right)_{j}
\end{array}\right] \tilde{X}_{t} \equiv F\left(p_{t}\right)_{j} \tilde{X}_{t}
$$


The value function for the original problem with $\tilde{X}_{t}$ given is

$$
\tilde{X}_{t}^{\prime} V\left(p_{t}\right) \tilde{X}_{t}+w\left(p_{t}\right)
$$

where the matrix function $V\left(p_{t}\right)$ and the scalar function $w\left(p_{t}\right)$ are determined in the following way: Note that we have

$$
\begin{gathered}
{\left[\begin{array}{c}
X_{t} \\
i_{t} \\
x_{t}
\end{array}\right]=\left[\begin{array}{c}
I \\
F_{i}\left(p_{t}\right) \\
F_{x}\left(p_{t}\right)_{j}
\end{array}\right] \tilde{X}_{t},} \\
\tilde{X}_{t+1}=M\left(p_{t}\right)_{j k} \tilde{X}_{t}+\tilde{C}_{k} \varepsilon_{t+1}, \\
M\left(p_{t}\right)_{j k} \equiv \tilde{A}_{k}+\hat{B}_{k} \hat{F}\left(p_{t}\right)_{j} .
\end{gathered}
$$

It follows that we can write the period loss as

$$
L_{t}=\tilde{X}_{t}^{\prime} \bar{W}\left(p_{t}\right)_{j} \tilde{X}_{t}
$$

where

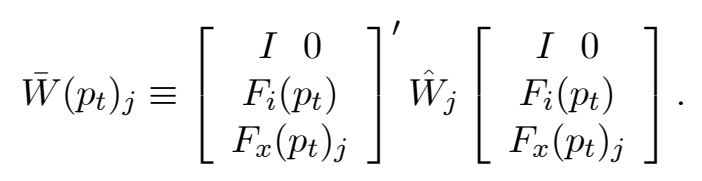

The matrix function $V\left(p_{t}\right)$ will then satisfy the Lyapunov function

$$
V\left(p_{t}\right)=\sum_{j} p_{t j}\left[\bar{W}\left(p_{t}\right)_{j}+\delta \sum_{k} P_{j k} M\left(p_{t}\right)_{j k}^{\prime} V\left(p_{t} P\right) M\left(p_{t}\right)_{j k}\right],
$$

and the function $w\left(p_{t}\right)$ will satisfy the equation ${ }^{22}$

$$
w\left(p_{t}\right)=\delta \sum_{j} \sum_{k} p_{t j} P_{j k}\left[\operatorname{tr}\left(V\left(p_{t} P\right) \tilde{C}_{k} \tilde{C}_{k}^{\prime}\right)+w\left(p_{t} P\right)\right]
$$

\section{I.2 An algorithm for the model with forward-looking variables}

Consider an algorithm for determining $F\left(p_{t}\right)_{j}$ and $V\left(p_{t}\right)$ in (I.2) and (I.4), respectively, for a given distribution of the modes in period $t, p_{t}$. In order to get a starting point for the iteration, we assume that the modes become observable $T+1$ periods ahead, that is, in period $t+T+1$. Hence, from that period on, the relevant solution is given by the matrices $F_{j}$ and $\tilde{V}_{j}$, where $F_{j}$ is the optimal policy function and $\tilde{V}_{j}$ is the value-function matrix for the dual saddlepoint problem with observable modes determined by the algorithm in appendix B. We consider these matrices and the horizon $T$ as known, and we will consider an iteration for $\tau=T, T-1, \ldots, 0$ that determines $F\left(p_{t}\right)_{j}$

\footnotetext{
${ }^{22}$ Note that $\tilde{C}_{k} \tilde{C}_{k}^{\prime}$ is the covariance matrix of the shocks $\tilde{C}_{k} \varepsilon_{t+1}$ to $\tilde{X}_{t+1}$ when $j_{t+1}=k(k=1, \ldots, n)$.
} 
and thereby $V\left(p_{t}\right)$ as a function of $T$. The horizon $T$ will then be increased until $F\left(p_{t}\right)_{j}$ and $V\left(p_{t}\right)$ converges. $^{23}$

Let $p_{t+\tau, t}$ for $\tau=0, \ldots, T$ and given $p_{t}$ be determined by (7.1), and let $\tilde{V}^{T+1}$ denote the modedependent matrices $\tilde{V}_{k}(k=1, \ldots, n)$ (or any arbitrary symmetric positive semidefinite matrix). Then, for $\tau=T, T-1, \ldots, 0$, let the mode-dependent matrix $F_{j}^{\tau}$ and the mode-independent matrix $\tilde{V}^{\tau}$ be determined recursively by

$$
\begin{aligned}
& J_{i i}^{\tau} \equiv \sum_{j} p_{t+\tau, j}\left[\hat{R}_{i i j}+\delta \sum_{k} P_{j k} \hat{B}_{\cdot i k}^{\prime} \tilde{V}^{\tau+1} \hat{B}_{\cdot i k}\right], \\
& J_{i j}^{\tau} \equiv p_{t+\tau, j}\left[\hat{R}_{i \tilde{x} j}+\delta \sum_{k} P_{j k} \hat{B}_{\cdot i k}^{\prime} \tilde{V}^{\tau+1} \hat{B}_{\cdot \tilde{x} k}\right] \quad(j=1, \ldots, n), \\
& J_{j i}^{\tau} \equiv \hat{R}_{\tilde{x} i j}+\delta \sum_{k} P_{j k} \hat{B}_{\cdot \tilde{x} k}^{\prime} \tilde{V}^{\tau+1} \hat{B}_{\cdot i k} \quad(j=1, \ldots, n), \\
& J_{j j}^{\tau} \equiv \hat{R}_{\tilde{x} \tilde{x} j}+\delta \sum_{k} P_{j k} \hat{B}_{\cdot \tilde{x} k}^{\prime} \tilde{V}^{\tau+1} \hat{B}_{\cdot \tilde{x} k} \quad(j=1, \ldots, n), \\
& J^{\tau} \equiv\left[\begin{array}{cccc}
J_{i i}^{\tau} & J_{i 1}^{\tau} & \cdots & J_{i n}^{\tau} \\
J_{1 i}^{\tau} & J_{11}^{\tau} & 0 & 0 \\
\vdots & 0 & \ddots & 0 \\
J_{n i}^{\tau} & 0 & 0 & J_{n n}^{\tau}
\end{array}\right] \\
& K^{\tau} \equiv\left[\begin{array}{c}
\sum_{j} p_{t+\tau, j}\left[\hat{N}_{\cdot i j}^{\prime}+\delta \sum_{k} P_{j k} \hat{B}_{\cdot i k}^{\prime} \tilde{V}^{\tau+1} \tilde{A}_{k}\right] \\
\hat{N}_{\cdot \tilde{x} 1}^{\prime}+\delta \sum_{k} P_{1 k} \hat{B}_{\cdot \tilde{x} k}^{\prime} \tilde{V}^{\tau+1} \tilde{A}_{k} \\
\vdots \\
\hat{N}_{\cdot \tilde{x} n}^{\prime}+\delta \sum_{k} P_{n k} \hat{B}_{\cdot \tilde{x} k}^{\prime} \tilde{V}^{\tau+1} \tilde{A}_{k}
\end{array}\right] \\
& \check{F}^{\tau} \equiv\left[\begin{array}{c}
F_{i}^{\tau} \\
F_{x 1}^{\tau} \\
F_{\gamma 1}^{\tau} \\
\vdots \\
F_{x n}^{\tau} \\
F_{\gamma n}^{\tau}
\end{array}\right]=-\left(J^{\tau}\right)^{-1} K^{\tau} \\
& \hat{F}_{j}^{\tau} \equiv\left[\begin{array}{c}
F_{i}^{\tau} \\
F_{x j}^{\tau} \\
F_{\gamma j}^{\tau}
\end{array}\right] \quad(j=1, \ldots, n) \\
& \tilde{V}^{\tau}=\sum_{j} p_{t+\tau, j}\left\{\begin{array}{l}
Q_{j}+\hat{N}_{j} \hat{F}_{j}^{\tau}+\hat{F}_{j}^{\tau \prime} \hat{N}_{j}^{\prime}+\hat{F}_{j}^{\tau \prime} \hat{R}_{j} \hat{F}_{j}^{\tau} \\
\left.+\delta \sum_{k} P_{j k}\left[\tilde{A}_{k}^{\prime}+\hat{F}_{j}^{\tau \prime} \hat{B}_{k}^{\prime}\right] \tilde{V}^{\tau+1}\left[\tilde{A}_{k}+\hat{B}_{k} \hat{F}_{j}^{\tau}\right)\right]
\end{array}\right\} .
\end{aligned}
$$

\footnotetext{
${ }^{23}$ It is obviously not necessary to assume that the modes become observable in some future period. We could instead let the iteration start far in the future with an arbitrary symmetric positive definite matrix instead of $\tilde{V}_{j}$.
} 
For $\tau=0, \ldots, T$, let

$$
\begin{gathered}
\bar{W}_{j}^{\tau} \equiv\left[\begin{array}{cc}
I & 0 \\
F_{i}^{\tau} \\
F_{x j}^{\tau}
\end{array}\right]^{\prime} \hat{W}_{j}\left[\begin{array}{cc}
I & 0 \\
F_{i}^{\tau} \\
F_{x j}^{\tau}
\end{array}\right], \\
M_{j k}^{\tau} \equiv \tilde{A}_{k}+\hat{B}_{k} \hat{F}_{j}^{\tau} .
\end{gathered}
$$

Let $V^{T+1}$ denote the mode-dependent value-function matrix $V_{j}$ for the original problem with forward-looking variables and observable modes. For $\tau=T, T-1, \ldots, 0$ define the mode-independent matrix $V^{\tau}$ recursively as

$$
V^{\tau}=\sum_{j} p_{t+\tau, j}\left[\bar{W}_{j}^{\tau}+\delta \sum_{k} P_{j k} M_{j k}^{\tau \prime} V^{\tau+1} M_{j k}^{\tau}\right] .
$$

This procedure will give $\hat{F}_{j}^{0}$ and $V^{0}$ as functions of $T$. We let $T$ increase until $\hat{F}_{j}^{0}$ and $V^{0}$ have converged. Then, the optimal policy function $F\left(p_{t}\right)_{j}$ and the matrix $V\left(p_{t}\right)$ are given by

$$
\begin{gathered}
F\left(p_{t}\right)_{j} \equiv\left[\begin{array}{c}
F_{x}\left(p_{t}\right)_{j} \\
F_{i}\left(p_{t}\right) \\
F_{\gamma}\left(p_{t}\right)_{j}
\end{array}\right]=\left[\begin{array}{c}
F_{x j}^{0} \\
F_{i}^{0} \\
F_{\gamma j}^{0}
\end{array}\right], \\
V\left(p_{t}\right)=V^{0} .
\end{gathered}
$$

\section{I.3 Unobservable modes without forward-looking variables}

When the model is backward-looking, the Bellman equation is,

$$
X_{t}^{\prime} V\left(p_{t}\right) X_{t}+w\left(p_{t}\right)=\min _{i_{t}}\left\{\begin{array}{l}
X_{t}^{\prime} Q\left(p_{t}\right) X_{t}+2 X_{t}^{\prime} N\left(p_{t}\right) i_{t}+i_{t}^{\prime} R\left(p_{t}\right) i_{t} \\
+\delta \sum_{j} \sum_{k} p_{t j} P_{j k}\left[X_{t+1, k}^{\prime} V\left(p_{t} P\right) X_{t+1, k}+w\left(p_{t} P\right)\right]
\end{array}\right\},
$$

where

$$
\begin{gathered}
W\left(p_{t}\right) \equiv\left[\begin{array}{cc}
Q\left(p_{t}\right) & N\left(p_{t}\right) \\
N\left(p_{t}\right)^{\prime} & R\left(p_{t}\right)
\end{array}\right] \equiv \sum_{j} p_{t j} W_{j} \equiv \sum_{j} p_{t j}\left[\begin{array}{cc}
Q_{j} & N_{j} \\
N_{j}^{\prime} & R_{j}
\end{array}\right], \\
X_{t+1, k}=A_{k} X_{t}+B_{k} i_{t}+C_{k} \varepsilon_{t+1} .
\end{gathered}
$$

The first-order condition with respect to $i_{t}$ is

$$
X_{t}^{\prime} N\left(p_{t}\right)+i_{t}^{\prime} R\left(p_{t}\right)+\delta \sum_{j} \sum_{k} p_{t j} P_{j k}\left[X_{t}^{\prime} A_{k}^{\prime} V\left(p_{t} P\right) B_{k}+i_{t}^{\prime} B_{k}^{\prime} V\left(p_{t} P\right) B_{k}\right]=0
$$

and can be written

$$
J\left(p_{t}\right) i_{t}+K\left(p_{t}\right) X_{t}=0
$$

where

$$
J\left(p_{t}\right) \equiv R\left(p_{t}\right)+\delta \sum_{j} \sum_{k} p_{t j} P_{j k} B_{k}^{\prime} V\left(p_{t} P\right) B_{k},
$$




$$
\left.K\left(p_{t}\right) \equiv N\left(p_{t}\right)^{\prime}+\delta \sum_{j} \sum_{k} p_{t j} P_{j k} B_{k}^{\prime} V\left(p_{t} P\right) A_{k}\right] .
$$

This leads to the optimal policy function

$$
i_{t}=F\left(p_{t}\right) X_{t}
$$

where

$$
F\left(p_{t}\right)=-J\left(p_{t}\right)^{-1} K\left(p_{t}\right)
$$

This implies the following Riccati equation for $V\left(p_{t}\right)$ :

$$
\begin{aligned}
V\left(p_{t}\right)= & Q\left(p_{t}\right)+N\left(p_{t}\right) F\left(p_{t}\right)+F\left(p_{t}\right)^{\prime} N\left(p_{t}\right)^{\prime}+F\left(p_{t}\right)^{\prime} R\left(p_{t}\right) F\left(p_{t}\right) \\
& +\delta \sum_{j} \sum_{k} p_{t j} P_{j k}\left[A_{k}^{\prime}+F\left(p_{t}\right)^{\prime} B_{k}^{\prime}\right] V\left(p_{t} P\right)\left[A_{k}+B_{k} F\left(p_{t}\right)\right] \\
= & Q\left(p_{t}\right)+\delta \sum_{j} \sum_{k} p_{t j} P_{j k} A_{k}^{\prime} V\left(p_{t} P\right) A_{k}-K\left(p_{t}\right)^{\prime} J\left(p_{t}\right)^{-1} K\left(p_{t}\right) .
\end{aligned}
$$

The scalar $w\left(p_{t}\right)$ solves the equation:

$$
w\left(p_{t}\right)=\delta \sum_{j} \sum_{k} p_{t j} P_{j k}\left[\operatorname{tr}\left(V\left(p_{t} P\right) C_{k} C_{k}^{\prime}\right)+w\left(p_{t} P\right)\right]
$$

\section{I.4 An algorithm for the backward-looking model}

For the backward-looking model, the algorithm can be written

$$
\begin{aligned}
J^{T} & \equiv \sum_{j} p_{t+T, j}\left[R_{j}+\delta \sum_{k} P_{j k} B_{k}^{\prime} V_{k} B_{k}\right], \\
K^{T} & \equiv \sum_{j} p_{t+T, j}\left[N_{j}^{\prime}+\delta \sum_{k} P_{j k} B_{k}^{\prime} V_{k} A_{k}\right], \\
V^{T} & =\sum_{j} p_{t+T, j}\left[Q_{j}+\delta \sum_{k} P_{j k} A_{k}^{\prime} V_{k} A_{k}\right]-\left(K^{T-1}\right)^{\prime}\left(J^{T-1}\right)^{-1} K^{T-1} .
\end{aligned}
$$

Given this, consider the iteration for $\tau=T-1, \ldots, 0$,

$$
\begin{aligned}
J^{\tau} & \equiv \sum_{j} p_{t+\tau, j}\left[R_{j}+\delta \sum_{k} P_{j k} B_{k}^{\prime} V^{\tau+1} B_{k}\right], \\
K^{l} & \equiv \sum_{j} p_{t+\tau, j}\left[N_{j}^{\prime}+\delta \sum_{k} P_{j k} B_{k}^{\prime} V^{l+1} A_{k}\right], \\
V^{l} & =\sum_{j} p_{t+\tau, j}\left[Q_{j}+\delta \sum_{k} P_{j k} A_{k}^{\prime} V^{l+1} A_{k}\right]-\left(K^{l}\right)^{\prime}\left(J^{l}\right)^{-1} K^{l} .
\end{aligned}
$$


Then, $T$ should be increased until $V^{0}$ converges. Then ${ }^{24}$

$$
\begin{aligned}
& V\left(p_{t}\right)=V^{0}, \\
& F\left(p_{t}\right)=-\left(J^{0}\right)^{-1} K^{0} .
\end{aligned}
$$

\section{J Optimization under discretion}

Here we also specify the equilibrium under discretionary optimization, that is, when the central bank cannot commit but reoptimizes each period. Oudiz and Sachs [29] derive an algorithm for the solution of this problem when there is no model uncertainty (and with $H=I$ ). This algorithm is further discussed in Backus and Driffill [3], Currie and Levin [12], Söderlind [32], and Svensson [37]. The algorithm is here adapted to the MJLQ framework. Blake and Zampolli [4] also provide an algorithm for the discretion equilibrium in the MJLQ framework.

Consider the central bank's decision problem to choose $i_{t}$ in period $t$ to minimize the intertemporal loss function (2.3) under discretion, that is, subject to (2.1), (2.2), and $X_{t}$ and $j_{t}$ given. ${ }^{25}$ Furthermore, the central bank anticipates that it will reoptimize in period $t+1$. That reoptimization will result in the instruments and the forward-looking variables in period $t+1$ being functions of the predetermined variables and the mode in period $t+1$ according to

$$
\begin{array}{rlr}
i_{t+1}=F_{t+1, j_{t+1}} X_{t+1} & \left(j_{t+1}=1, \ldots, n\right), \\
x_{t+1}=G_{t+1, j_{t+1}} X_{t+1} & \left(j_{t+1}=1, \ldots, n\right) .
\end{array}
$$

The reoptimization will also result in value of the problem in period $t+1$,

$$
X_{t+1}^{\prime} V_{t+1, j_{t+1}} X_{t+1}+w_{t+1, j_{t}+1} \quad\left(j_{t+1}=1, \ldots, n\right) .
$$

For any $t$, we here let $F_{t} \equiv\left\{F_{t j}\right\}_{j=1}^{n}, G_{t} \equiv\left\{G_{t j}\right\}_{j=1}^{n}$, and $V_{t} \equiv\left\{V_{t j}\right\}_{j=1}^{n}$ denote the set of matrices $F_{t j}(j=1, \ldots, n), G_{t j}(j=1, \ldots, n)$, and $V_{t j}(j=1, \ldots, n)$, respectively. We assume that the set of matrices $F_{t+1}, G_{t+1}$, and $V_{t+1}$ in period $t+1$ are known by the central bank in period $t$. We will see that optimization in period $t$ will then determine the set of matrices $F_{t}, G_{t}$, and $V_{t}$ in period $t$ as a function of the set of matrices $G_{t+1}$ and $V_{t+1}$. This specifies a mapping of $\left(G_{t+1}, V_{t+1}\right)$ to $\left(G_{t}, V_{t}\right)$.

\footnotetext{
${ }^{24}$ A related paper is do Val and Başar [13], who consider the problem of "receding horizon control." They introduce a terminal payoff, and at each date $t$ they solve a finite-horizon optimization problem looking ahead $T$ periods given the current probability distribution. The action taken at the current date is then the first optimal choice in the solution of the finite horizon problem. Then the distribution is updated and the problem repeats.

${ }_{25}$ That is, we assume that the modes are observable. The algorithm is easily modified to the case when the modes are unobservable.
} 
We are looking for a fixed point of this mapping. We denote the fixed point by $G \equiv\left\{G_{j}\right\}_{j=1}^{n}$ and $V \equiv\left\{V_{j}\right\}_{j=1}^{n}$.

First, by (J.2) and (2.1) we have,

$$
\begin{aligned}
\mathrm{E}_{t} H_{t+1} x_{t+1} & =\mathrm{E}_{t} H_{t+1} G_{t+1} X_{t+1} \\
& =\mathrm{E}_{t} H_{t+1} G_{t+1}\left(A_{11, t+1} X_{t}+A_{12, t+1} x_{t}+B_{1, t+1} i_{t}\right)
\end{aligned}
$$

(where $\mathrm{E}_{t} H_{t+1} G_{t+1} X_{t+1}$ denotes $\sum_{k=1}^{n} P_{j_{t} k} H_{k} G_{t+1, k} X_{t+1, k}$ conditional on a given $j_{t}=1, \ldots, n$ and $X_{t+1, k}$ refers to the realization of $X_{t+1}$ when $j_{t+1}=k$ ). Combining this with (2.2) gives

$$
\mathrm{E}_{t} H_{t+1} G_{t+1}\left(A_{11, t+1} X_{t}+A_{12, t+1} x_{t}+B_{1, t+1} i_{t}\right)=A_{21, t} X_{t}+A_{22, t} x_{t}+B_{2, t} i_{t} .
$$

Solving for $x_{t}$ gives

$$
x_{t}=\bar{A}_{t j_{t}} X_{t}+\bar{B}_{t j_{t}} i_{t} \quad\left(j_{t}=1, \ldots, n\right)
$$

where

$$
\begin{aligned}
\bar{A}_{t j} & \equiv\left(A_{22 j}-\mathrm{E}_{t} H_{t+1} G_{t+1} A_{12, t+1}\right)^{-1}\left(\mathrm{E}_{t} H_{t+1} G_{t+1} A_{11, t+1}-A_{21, t}\right), \\
\bar{B}_{t} & \equiv\left(A_{22 j}-\mathrm{E}_{t} H_{t+1} G_{t+1} A_{12, t+1}\right)^{-1}\left(\mathrm{E}_{t} H_{t+1} G_{t+1} B_{1, t+1}-B_{2, t}\right)
\end{aligned}
$$

(we assume that $A_{22, t}-\mathrm{E}_{t} H_{t+1} G_{t+1} A_{12, t+1}$ is invertible). Using (J.3) in (2.1) then gives

$$
X_{t+1}=\tilde{A}_{t+1} X_{t}+\tilde{B}_{t+1} i_{t}+C_{t+1} \varepsilon_{t+1},
$$

where

$$
\begin{aligned}
\tilde{A}_{t+1} & \equiv A_{11, t+1}+A_{12, t+1} \bar{A}_{t} \\
\tilde{B}_{t+1} & \equiv B_{1, t+1}+A_{12 t+1} \bar{B}_{t} .
\end{aligned}
$$

Third, using (J.3) in (2.4) gives

$$
L_{t}=\left[\begin{array}{c}
X_{t} \\
i_{t}
\end{array}\right]^{\prime}\left[\begin{array}{cc}
Q_{t} & N_{t} \\
N_{t}^{\prime} & R_{t}
\end{array}\right]\left[\begin{array}{c}
X_{t} \\
i_{t}
\end{array}\right]
$$

where

$$
\begin{aligned}
Q_{t} & \equiv W_{X X, t}+W_{X x, t} \bar{A}_{t}+\bar{A}_{t}^{\prime} W_{X x, t}^{\prime}+\bar{A}_{t}^{\prime} W_{x x, t} \bar{A}_{t} \\
N_{t} & \equiv W_{X x, t} \bar{B}_{t}+\bar{A}_{t}^{\prime} W_{x x, t} \bar{B}_{t}+W_{X i, t}+\bar{A}_{t}^{\prime} W_{x i, t}, \\
R_{t} & \equiv W_{i i, t}+\bar{B}_{t}^{\prime} W_{i i, t} \bar{B}_{t}+\bar{B}_{t}^{\prime} W_{x i, t}+W_{x i, t}^{\prime} \bar{B}_{t}
\end{aligned}
$$


and $W_{t}$ is partitioned conformably with $X_{t}, x_{t}$, and $i_{t}$,

$$
W_{t} \equiv\left[\begin{array}{ccc}
W_{X X, t} & W_{X x, t} & W_{X i, t} \\
W_{x X, t} & W_{x x, t} & W_{x i, t} \\
W_{i X, t} & W_{i x, t} & W_{i i, t}
\end{array}\right] .
$$

Fourth, the value of the problem in period $t$ is associated with the symmetric positive semidefinite matrix $V_{t}$ and the scalar $w_{t}$, and it satisfies the Bellman equation

$$
X_{t}^{\prime} V_{t j} X_{t}+w_{t j} \equiv \min _{i_{t}}\left\{L_{t j}+\delta \mathrm{E}\left[X_{t+1}^{\prime} V_{t+1} X_{t+1}+w_{t+1} \mid j_{t}=j\right]\right\} \quad(j=1, \ldots, n)
$$

subject to (J.6) and (J.9). Indeed, the problem has been transformed to a MJLQ problem without forward-looking variables, albeit with time-varying coefficients. The first-order condition is, by (J.9) and (J.13),

$$
\begin{aligned}
0 & =X_{t}^{\prime} N_{t j}+i_{t}^{\prime} R_{t j}+\delta \mathrm{E}\left[X_{t+1}^{\prime} V_{t+1} \tilde{B}_{t+1} \mid j_{t}=j\right] \\
& =X_{t}^{\prime} N_{t j}+i_{t}^{\prime} R_{t j}+\delta \mathrm{E}\left[\left(X_{t}^{\prime} \tilde{A}_{t+1}^{\prime}+i_{t}^{\prime} \tilde{B}_{t+1}^{\prime}\right) V_{t+1} \tilde{B}_{t+1} \mid j_{t}=j\right] \quad(j=1, \ldots, n) .
\end{aligned}
$$

The first-order condition can be solved for the policy function,

$$
i_{t}=F_{t j_{t}} X_{t} \quad\left(j_{t}=1, \ldots, n\right),
$$

where

$$
\begin{gathered}
F_{t j} \equiv-J_{t j}^{-1} K_{t j} \\
J_{t j} \equiv R_{t j}+\delta \mathrm{E}\left[\tilde{B}_{t+1}^{\prime} V_{t+1} \tilde{B}_{t+1} \mid j_{t}=j\right] \\
K_{t j} \equiv N_{t j}^{\prime}+\delta \mathrm{E}\left[\tilde{B}_{t+1}^{\prime} V_{t+1} \tilde{A}_{t+1} \mid j_{t}=j\right]
\end{gathered}
$$

(we assume that $J_{t j}$ is invertible). Using (J.14) in (J.3) gives

$$
x_{t}=G_{t j_{t}} X_{t} \quad\left(j_{t}=1, \ldots, n\right),
$$

where

$$
G_{t j} \equiv \bar{A}_{t j}+\bar{B}_{t j} F_{t j}
$$

Furthermore, using (J.14) in (J.13) and identifying terms result in

$$
\begin{aligned}
V_{t j} & \equiv Q_{t j}+N_{t j} F_{t j}+F_{t j}^{\prime} N_{t j}^{\prime}+F_{t j}^{\prime} R_{t j} F_{t j}+\delta \mathrm{E}\left[\left(\tilde{A}_{t+1}+\tilde{B}_{t+1} F_{t}\right)^{\prime} V_{t+1}\left(\tilde{A}_{t+1}+\tilde{B}_{t+1} F_{t}\right) \mid j_{t}=j\right] \\
& \equiv Q_{t j}+\delta \mathrm{E}\left[\tilde{A}_{t+1}^{\prime} V_{t+1} \tilde{A}_{t+1} \mid j_{t}=j\right]-K_{t j}^{\prime} J_{t j}^{-1} K_{t j} \quad(j=1, \ldots, n) .
\end{aligned}
$$


Thus, the above equations (J.4), (J.5), (J.7), (J.8), (J.10)-(J.12), (J.15), (J.16), and (J.17)) define a mapping from the set of matrices $\left(G_{t+1}, V_{t+1}\right)$ to the set of matrices $\left(G_{t}, V_{t}\right)$. This mapping also determines the set of matrices $F_{t}$. The discretion equilibrium is a fixed point $(G, V) \equiv\left\{G_{j}, V_{j}\right\}_{j=1}^{n}$ of the mapping and a corresponding $F \equiv\left\{F_{j}\right\}_{j=1}^{n}$. The fixed point can be obtained as the limit of $\left(G_{t}, V_{t}\right)$ when $t \rightarrow-\infty$.

The details of this algorithm can be completed in the same way as for our other algorithms. The algorithm is easily generalized to the case when the modes are not observable. 


\section{References}

[1] Anderson, Evan W., Lars Peter Hansen, Ellen R. McGrattan, and Thomas J. Sargent (1995), "Mechanics of Forming and Estimating Dynamic Linear Economies," working paper, homepages.nyu.edu $/ \sim$ ts $43 /$.

[2] Aoki, Masanao (1967), Optimization of Stochastic Systems, Academic Press, New York.

[3] Backus, David, and John Driffill (1986), "The Consistency of Optimal Policy in Stochastic Rational Expectations Models," CEPR Discussion Paper No. 124.

[4] Blake, Andrew P., and Fabrizio Zampolli (2005), "Time Consistent Policy in Markov Switching Models," working paper, Bank of England.

[5] Blinder, Alan S., and Ricardo Reis (2005), "Understanding the Greenspan Standard," in The Greenspan Era: Lessons for the Future, a symposium sponsored by the Federal Reserve Bank of Kansas City, forthcoming, www.federalreserve.gov.

[6] Brainard, W. (1967), "Uncertainty and the Effectiveness of Policy," American Economic Review 57, 411-425.

[7] Brash, Donald T. (2000), "Making Monetary Policy: A Look Behind the Curtains," speech in Christchurch, January 26, 2000, Reserve Bank of New Zealand, www.rbnz.govt.nz.

[8] Chow, Gregory C. (1973) "Effect of Uncertainty on Optimal Control Policies," International Economic Review 14, 632-45.

[9] Cogley, Timothy, Riccardo Colacito and Thomas J. Sargent (2005) "The Benefits from U.S. Monetary Policy Experimentation in the Days of Samuelson and Solow and Lucas," working paper, NYU.

[10] Costa, Oswaldo L.V., and Marcelo D. Fragoso (1995), "Discrete-Time LQ-Optimal Control Problems for Infinite Markov Jump Parameter Systems," IEEE Transactions on Automatic Control 40, 2076-2088.

[11] Costa, Oswaldo L.V., Marecelo D. Fragoso, and Ricardo P. Marques (2005), Discrete-Time Markov Jump Linear Systems, Springer, London. 
[12] Currie, David, and Paul Levine (1993), Rules, Reputation and Macroeconomic Policy Coordination, Cambridge University Press, Cambridge.

[13] do Val, João B.R., and Tamer Başar (1999), "Receding Horizon Control of Jump Linear Systems and a Macroeconomic Policy Problem," Journal of Economic Dynamics and Control 23, 1099-1131.

[14] do Val, João B.R, José C. Geromel, and Oswaldo L.V. Costa (1998), "Uncoupled Riccati Iterations for the Linear Quadratic Control Problem of Discrete-Time Markov Jump Linear Systems," IEEE Transactions on Automatic Control 43, 1727-1733.

[15] Feldstein, Martin (2004), "Innovations and Issues in Monetary Policy: Panel Discussion," American Economic Review 94 (May) 41-43.

[16] Gantmacher, Felix R. (1959), The Theory of Matrices, Volume II, Chelsea Publishing Company, New York.

[17] Greenspan, Alan (2004), "Risk and Uncertainty in Monetary Policy," American Economic Review 94 (May) 33-40.

[18] Greenspan, Alan (2005), "Reflections on Central Banking," in The Greenspan Era: Lessons for the Future, a symposium sponsored by the Federal Reserve Bank of Kansas City, forthcoming, www.federalreserve.gov.

[19] Hamilton, James D. (1994), Time Series Analysis, Princeton University Press.

[20] Kahn, James A. and Robert W. Rich (2004) "Tracking the New Economy: Using Growth Theory to Detect Changes in Trend Productivity," working paper, Federal Reserve Bank of New York.

[21] Karlin, Samuel, and Howard M. Taylor (1975), A First Course in Stochastic Processes, 2nd edition, Academic Press, San Diego, CA.

[22] Kim, Chang-Jin and Charles R. Nelson (1999), State-Space Models with Regime Switching, MIT Press, Cambridge, MA.

[23] Levin, Andrew T., Alexei Onatski, John C. Williams, and Noah Williams (2005), "Monetary Policy Under Uncertainty in Micro-Founded Macroeconometric Models," working paper for the 2005 NBER Macroeconomics Annual. 
[24] Lindé, Jesper (2002), "Estimating New-Keynesian Phillips Curves: A Full Information Maximum Likelihood Approach," Sveriges Riksbank Working Paper Series No. 129, www.riksbank.se.

[25] Ljungqvist, Lars, and Thomas J. Sargent (2005), Recursive Macroeconomic Theory, 2nd edition, MIT Press, Cambridge, MA.

[26] Marcet, Albert, and Ramon Marimon (1998), "Recursive Contracts," working paper, www.econ.upf.edu.

[27] McConnell, Margaret M. and Gabriel Perez-Quiros (2000) "Output Fluctuations in the United States: What Has Changed since the Early 1980's?", American Economic Review 90, 1464-76.

[28] Onatski, Alexei, and Noah Williams (2003), "Modeling Model Uncertainty," Journal of the European Economic Association 1, 1087-1022.

[29] Oudiz, Gilles, and Jeffrey Sachs (1985), "International Policy Coordination in Dynamic Macroeconomic Models," in William H. Buiter and Richard C. Marston, eds., International Economic Policy Coordination, Cambridge University Press, Cambridge.

[30] Rudebusch, Glenn D., and Lars E.O. Svensson (1999), "Policy Rules for Inflation Targeting," in John B. Taylor (ed.), Monetary Policy Rules, University of Chicago Press.

[31] Sims, Christopher A. (2002), "The Role of Models and Probabilities in the Monetary Policy Process," Brookings Papers on Economic Activity 2:2002, 1-62.

[32] Söderlind, Paul (1999), "Solution and Estimation of RE Macromodels with Optimal Policy," European Economic Review 43, 813-823.

[33] Stock, James H. and Mark W. Watson (2002) "Has the Business Cycle Changed and Why?" in Mark Gertler and Ken Rogoff, eds., NBER Macroeconomics Annual 2002, MIT Press, Cambridge.

[34] Svensson, Lars E.O. (2001a), Independent Review of the Operation of Monetary Policy in New Zealand: Report to the Minister of Finance, www.princeton.edu/ svensson.

[35] Svensson, Lars E.O. (2001b), "Price Stability as a Target for Monetary Policy: Defining and Maintaining Price Stability," in Deutsche Bundesbank, ed., The Monetary Transmis- 
sion Process: Recent Developments and Lessons for Europe, Palgrave, New York, 60-102, www.princeton.edu/ svensson.

[36] Svensson, Lars E.O. (2005a), "Monetary Policy with Judgment: Forecast Targeting," International Journal of Central Banking 1, 1-54, www.ijcb.org.

[37] Svensson, Lars E.O. (2005b), "Optimization under Commitment and Discretion, the Recursive Saddlepoint Method, and Targeting Rules and Instrument Rules: Lecture Notes," www.princeton.edu/ svensson.

[38] Svensson, Lars E.O., and Robert J. Tetlow (2005), "Optimal Policy Projections," International Journal of Central Banking, forthoming, www.princeton.edu/ svensson.

[39] Svensson, Lars E.O., and Michael Woodford (2005), "Implementing Optimal Policy through Inflation-Forecast Targeting," in Bernanke, Ben S., and Michael Woodford, eds., Inflation Targeting, University of Chicago Press, www.princeton.edu/ svensson.

[40] Woodford, Michael (2003), Interest and Prices: Foundations of a Theory of Monetary Policy, Princeton University Press.

[41] Zampolli, Fabrizio (2005), "Optimal Monetary Policy in a Regime-Switching Economy: The Response to Abrupt Shifts in Exchange-Rate Dynamics," working paper, Bank of England. 